\author{
UNIVERSIDADE DE SÃO PAULO \\ ESCOLA DE ENGENHARIA DE SÃO CARLOS
}

CHARLES JASTER DE OLIVEIRA

ANÁLISE DA INTERAÇÃO PLACA-VIGA VIA MÉTODO DOS ELEMENTOS DE
CONTORNO

SÃO CARLOS

2012 

CHARLES JASTER DE OLIVEIRA

\section{ANÁLISE DA INTERAÇÃO PLACA-VIGA VIA MÉTODO DOS ELEMENTOS DE CONTORNO}

Dissertação apresentada à Escola de Engenharia de São Carlos, da Universidade de São Paulo, como parte dos requisitos para a obtenção do Título de Mestre em Engenharia de Estruturas.

Orientador: Prof. Dr. João Batista de Paiva. 
AUTORIZO A REPRODUÇÃO E DIVULGAÇÃO TOTAL OU PARCIAL DESTE TRABALHO, POR QUALQUER MEIO CONVENCIONAL OU ELETRÔNICO, PARA FINS DE ESTUDO E PESQUISA, DESDE QUE CITADA A FONTE.

Ficha catalográfica preparada pela Seção de Tratamento da Informação do Serviço de Biblioteca - EESC/USP

\begin{tabular}{|c|c|}
\hline \multirow[t]{3}{*}{$046 a$} & $\begin{array}{l}\text { Oliveira, Charles Jaster de } \\
\text { Análise da interação placa-viga via método dos } \\
\text { elementos de contorno / Charles Jaster de Oliveira ; } \\
\text { orientador João Batista de Paiva. -- São Carlos, } 2012 \text {. }\end{array}$ \\
\hline & $\begin{array}{l}\text { Dissertação (Mestrado - Programa de Pós-Graduação em } \\
\text { Engenharia de Estruturas) -- Escola de Engenharia de São } \\
\text { Carlos da Universidade de São Paulo, } 2012 \text {. }\end{array}$ \\
\hline & $\begin{array}{l}\text { 1. Método dos elementos de contorno. 2. Interação } \\
\text { placa-viga. 3. Interação laje-viga. 4. Pavimentos de } \\
\text { edifícios. I. Título. }\end{array}$ \\
\hline
\end{tabular}


Candidata: Engenheiro CHARLES JASTER DE OLIVEIRA.

Título da dissertação: "Análise da interação placa-viga via método dos elementos de contorno".

Data da defesa: 27/07/2012

\section{Comissão Julgadora:}

Prof. Associado João Batista de Paiva (Orientador)

(Escola de Engenharia de São Carlos/EESC)

Prof. Titular Humberto Breves Coda

(Escola de Engenharia de São Carlos/EESC)

Prof. Dr. Leandro Palermo Junior

(Universidade Estadual de Campinas/UNICAMP)

\section{Resultado:}



Aprovado

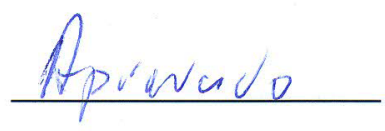

Coordenador do Programa de Pós-Graduação em Engenharia Civil (Engenharia de Estruturas):

Profa. Associada Ana Lucia Homce de Cresce El Debs

Presidente da Comissão de Pós-Graduação:

Prof. Titular Denis Vinicius Coury 

Dedico este trabalho a Deus, que nunca desistiu de mim e se manteve ao meu lado por todo este caminho. Aos meus pais e a minha amada noiva. 

Agradeço primeiramente a Deus.

Aos meus pais Herta Jaster de Oliveira e Jailson Monteiro de Oliveira por não terem poupado amor ao me criarem, agradeço por cada hora trabalhada para pagarem meus estudos, agradeço por terem me ensinado o único e bom caminho, pois é primordialmente pelos senhores que eu conquistei mais esta vitória.

Ao meu orientador João Batista de Paiva, pela compreensão, paciência e por todo conhecimento que me foi passado nestes anos.

Agradeço a minha amada noiva, Luna Ollin Steffen por todos esses anos cheios de desafios e conquistas que passamos, por ser parte da minha vitória. Agradeço por tudo, principalmente seu amor.

Agradeço aos meus irmãos Weece Jaster de Oliveira e Winderson Jaster de Oliveira.

A minha sogra Sânia e meus cunhados João Pedro e Luis Gustavo Steffen.

In memoriam, agradeço meu sogro Cícero Steffen que se orgulhava de mim e infelizmente se foi no meio desta jornada.

Aos amigos, em especial ao Rodrigo Pagnussat que sempre esteve presente quando eu precisei e por dividir tanto os momentos bons quanto os ruins comigo. A Erica Kimura por ser tão prestativa e atenciosa desde a minha entrada no SET até o ultimo dia, sem poupar simpatia. As queridas Indara e Orieta por todo carinho e sorrisos compartilhados.

Aos colegas que junto comigo ingressaram neste desafio: Emerson, Pedro, Rafael Tamanini, Rafael Eclache, Davi, Eunice, Juliana, Fabrício, Winston, Daniel, Marcelo, Nero, André, Luiz, Markus.

Aos amigos de outras turmas: Carlos, Ellen, Luiz, Hidelbrando, Wellison, Higor, Wagner, Jonas, Danielle, André Ramos, Érica, Marcela Filizola, Denis, Jesus Daniel, Jesus Sánchez, Aref, Dorival, Hugo.

Ao Conselho Nacional de Desenvolvimento Científico e Tecnológico, CNPq, pelo apoio financeiro para a realização desta pesquisa.

A todos os professores da graduação e da pós-graduação que contribuíram por essa minha paixão por estruturas.

A todos aqueles que direta ou indiretamente fizeram parte deste trabalho. 

OLIVEIRA, C. J. (2012). Análise da interação placa-viga via Método dos Elementos de Contorno. Dissertação (Mestrado) - Escola de Engenharia de São Carlos, Universidade de São Paulo, São Carlos.

Este trabalho apresenta o estudo de uma formulação do método dos elementos de contorno para a análise da interação placa-viga de pavimentos de edifícios. Para compatibilizar adequadamente as vigas e a placa, é utilizada uma formulação alternativa do método dos elementos de contorno para placas delgadas em que, a viga é modelada por equações diferenciais ordinárias e, a compatibilidade entre a placa e a viga é feita por meio dos deslocamentos e forças de ligação nos nós dos elementos. Após a imposição das condições de contorno e resolução final do sistema de equações, são obtidos os esforços nas vigas e deslocamentos na placa, que por fim são confrontados com outras formulações. Exemplos foram analisados demonstrando uma ótima concordância com os exemplos obtidos por outras formulações.

Palavras-chave: Método dos Elementos de Contorno, interação placaviga, interação laje-viga, pavimentos de edifícios. 

OLIVEIRA, C. J. (2012). Analysis of plate-beam interaction by the Boundary Element Method. Thesis (MA) - School of Engineering of São Carlos, Universidade de São Paulo, São Carlos.

This work presents a formulation of the boundary element method for the analysis of plate-beam interaction in buildings floors. In order to properly match the beams and plate is used an alternative formulation of the boundary element method for thin plates, in which the beam is modeled by ordinary differential equations and the compatibility between plate and beam is done by the displacements and forces of connection in the nodes of the elements. After applying boundary conditions and final resolution of the system of equations, efforts are obtained in beams and plate displacements, that are confronted with other formulations. Samples were analyzed showing excellent results compared with those obtained by other formulations.

Keywords: Boundary Element Method, plate-beam interaction, interaction slab-beam; building slabs. 



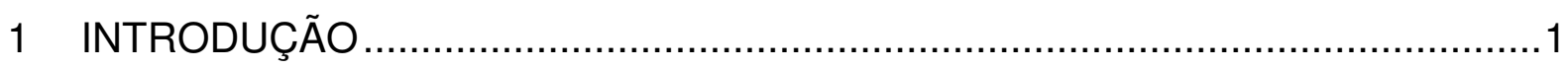

2 FUNDAMENTOS DA TEORIA DE PLACAS DELGADAS ....................................

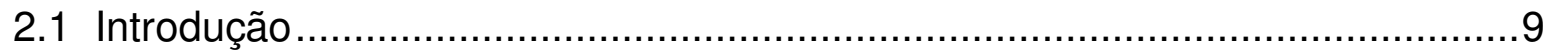



2.3 Equações de placas em coordenadas polares ….......................................16

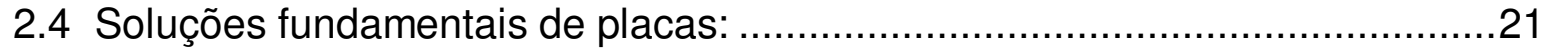

3 EQUAÇÕES INTEGRAIS PARA FLEXÃO DE PLACAS .....................................

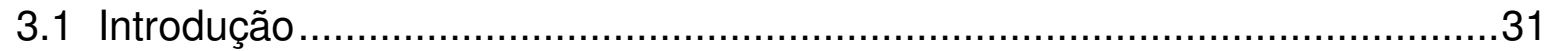

3.2 Equação integral para um ponto do domínio .................................................31

3.3 Equação integral para um ponto do contorno .................................................39

3.4 Transformações das integrais de domínio ....................................................53

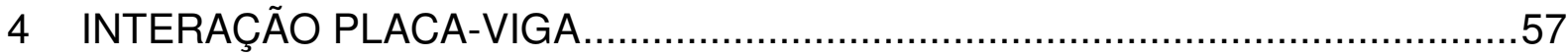

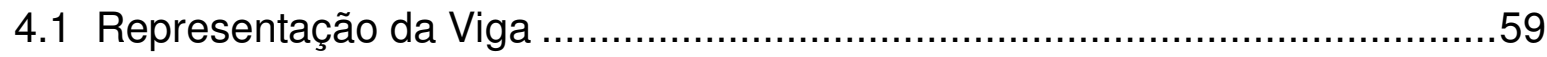



5 AVALIAÇÃO NUMÉRICA DA FORMULAÇÃO PROPOSTA...............................67

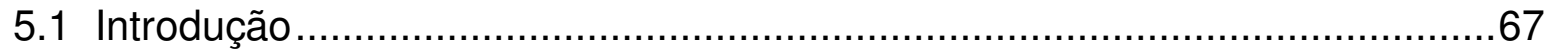

5.2 Exemplo 1 - Placa quadrada apoiada nos quatro cantos e vigas ao longo dos lados, com carregamento uniforme.

5.3 Exemplo 2 - Placa retangular apoiada nos quatro cantos e vigas ao longo dos lados e uma viga de domínio, com carregamento uniforme.

5.4 Exemplo 3 - Placa quadrada apoiada nos quatro cantos e vigas ao longo dos lados e do domínio, com carregamento uniforme.

5.5 Exemplo 4 - Pavimento de um edifício com vigas ao longo dos lados e do domínio, com carregamento uniforme.

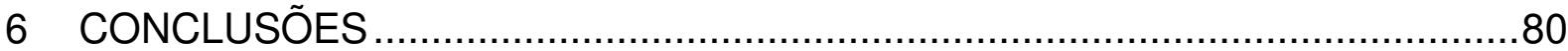

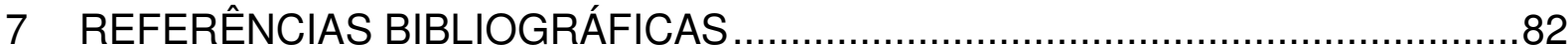



A Engenharia Civil possui diversos ramos de atuação com sua específica representatividade dentro de sua função. Um dos ramos é a Engenharia Estrutural que representa o segmento da engenharia responsável pelo estudo dos elementos e sistemas estruturais que compõe as obras da Engenharia Civil.

A Engenharia de Estruturas parte do princípio da concepção estrutural, pré-dimensionamento, partindo para a análise estrutural e culminando no dimensionamento do elemento estrutural. Os elementos estruturais que são estudados na Engenharia estrutural são de modo geral: vigas, placas, pilares, cascas, chapas, cabos, barras etc. Quando combinados, formam sistemas estruturais usuais e de notória importância no dia a dia da Engenharia Civil.

Um sistema estrutural típico e abordado neste trabalho é o sistema estrutural de um pavimento de edifício, que apresenta em sua configuração de elementos, placas e vigas. As vigas são elementos lineares e as placas, por definição, são elementos estruturais planos que estão sujeitos a um carregamento transversal ao seu plano médio.

Para a análise de uma estrutura de placa, pode-se considerar duas teorias fundamentais para o conhecimento e domínio do assunto, sendo estas a teoria clássica de KIRCHHOFF (1850) e as teorias de REISSNER (1944) e MINDLIN(1951).

A teoria de placas segundo KIRCHHOFF (1850) serviu de base para desenvolvimento deste trabalho. Na teoria de KIRCHHOFF (1850) são analisadas apenas placas delgadas, onde as hipóteses simplificadoras consideram quatro variáveis de contorno, levando a solução do problema a uma equação diferencial de quarto grau (quarta ordem). A Teoria de REISSNER (1944) e MINDLIN (1951) é consequência da existência de placas que não podem ser consideradas finas para as quais a contribuição da força cortante podem ser significativos (permitindo a análise de placas espessas). Como consequência, a solução do problema leva a uma equação diferencial de sexto grau (sexta ordem). Para este tipo de placas, as 
hipóteses de KIRCHHOFF (1850) consideradas válidas para as placas finas deixam de ser admissíveis.

Ao buscar as soluções dessas equações por meios analíticos, o processo se torna limitado, fazendo-se necessário o uso de métodos numéricos. Hoje, com a evolução tecnológica dos computadores, permite-se o uso de modelos melhor elaborados com considerações que vão além das considerações simplificadas das soluções analíticas, assim, fornecendo cálculos mais precisos para a representação do comportamento estrutural.

Os métodos numéricos aplicados para solução dos problemas envolvendo placas são: o Método das Diferenças Finitas (M.D.F.) e o Método dos Elementos Finitos (M.E.F.). Tanto o M.D.F. quanto o M.E.F., são métodos de domínio, em que a discretização é feita com elementos no domínio do problema (Figura 1.1). O Método dos Elementos de Contorno (M.E.C.), é um método de superfície que fundamentase na discretização apenas do contorno do problema (Figura 1.1). O M.E.C. é uma ferramenta muito útil nas modelagens numéricas, sua principal vantagem diante dos demais métodos é devido à simplicidade da geração das malhas dos modelos e à aplicação em problemas consistindo de domínios infinitos ou semi-infinitos.



Figura 1.1 - Métodos numéricos aplicados aos problemas de Engenharia. (KANE, 1994)

O Método das Diferenças Finitas (M.D.F.) é o mais antigo desses métodos e teve sua sistematização com o trabalho de SOUTHWELL (1946), sendo utilizado ainda hoje. O método transforma equações diferenciais em equações algébricas válidas apenas nos nós no domínio, através de aproximações das derivadas por diferenças finitas. 
Já o Método dos Elementos Finitos (M.E.F.), o mais utilizado dos métodos, transforma o próprio domínio em uma série de subdomínios finitos ou elementos conectados através de seus nós cujas variáveis sobre cada elemento são governadas por funções aproximadoras contínuas. Seu desenvolvimento teve início com os trabalhos de TURNER (1956) e ARGYRIS e KELSET (1960).

O Método dos Elementos de Contorno (M.E.C.), foco deste trabalho, surgiu decorrente do estudo de problemas utilizando equações integrais aplicadas a teoria da elasticidade por BETTI (1872).

MUSKELISHVILI (1953), MIKHLIN (1957) e KUPRADZE (1965) deram enorme contribuição utilizando equações integrais na elasticidade bidimensional, não utilizando-se ainda, as variáveis reais do problema (denominando-se, portanto de formulações indiretas). RIZZO (1967) foi o primeiro que tratou as equações integrais como forma de técnica numérica e também foi o primeiro a propor a formulação direta para o tratamento das equações integrais do problema elástico bidimensional.

Os primeiros trabalhos relacionados a análise de placas utilizando o Método dos Elementos de Contorno (M.E.C.) foram desenvolvidos por JASWON et al. (1967) considerando o MEC na sua forma indireta. O Método dos Elementos de Contorno (M.E.C.) na sua forma direta para a análise de placas infinitas com furos de contorno não carregado foi proposta por HANSEN (1976), e teve sequência nos estudos de BEZINE (1978), BEZINE e GAMBY (1978) e STERN (1979) baseando-se na identidade de Green e considerando como variáveis o deslocamento transversal e sua derivada na direção normal ao contorno. Nesta formulação foram deduzidas duas equações integrais relativas às variáveis utilizadas. A comparação entre as formulações direta e indireta foi apresentada por TOTTENHAM (1979). Para análise de placas espessas, VAN DER WEEËN (1982) propôs uma formulação baseado na teoria de Reissner, escrevendo três equações integrais para as variáveis nodais, deslocamento transversal e as derivadas nas direções normal e tangencial do contorno.

A partir dessas formulações básicas, formulações direta e indireta e, usando-se as teorias de placas de KIRCHHOFF (1850) e de REISSNER (1944) e MINDLIN (1951), diversas análises de placas se seguiram conforme exposto em OLIVEIRA NETO (1998): 
- Estudos para aperfeiçoamento numérico do método como também sobre quais equações integrais são utilizadas, estudos do deslocamento transversal e de sua derivada direcional ou apenas de deslocamento transversal para pontos distintos;

- Estudos sobre o melhor posicionamento do ponto-fonte, ao qual as equações correspondem;

- Estudos sobre os tipos de integração mais adequados (analítica ou numérica) e sobre as funções aproximadoras das variáveis sobre os elementos;

- Formas de cálculo de integrais singulares e quase singulares e, de integrais de domínio; análise de placas sob diversos tipos de vinculação e de carregamento, apoiadas sobre base elástica e sobre estacas;

- Análise da associação com vigas e colunas no contorno e no domínio; análise não-linear, física e geométrica;

- Vibração (análise modal e transiente);

- Placas com deslocamentos finitos;

- Combinação do M.E.C. com o M.D.F. e com o M.E.F. como também com outras formulações matemáticas para diversas análises aproveitando as vantagens apresentadas por cada método.

Apesar de ser a menos utilizada, a formulação indireta é objeto de estudos nos trabalhos de VABLE e ZHANG (1992), utilizando-se de integração analítica e funções fictícias aproximadas por polinômios para análise de placas e, o trabalho de VENTSEL (1997), com integrais singulares e hipersingulares.

KARAM (1986), BARCELLOS e SILVA (1987), LONG et al. (1988), WESTPHAL E BARCELLOS (1989) e RIBEIRO e VENTURINI (1989), basearam-se na teoria de REISSNER (1944) e MINDLIN (1951) para o desenvolvimento de seus estudos.

A consideração do comportamento não-linear geométrico de placas pela teoria de REISSNER foi analisado por XIAO-YAN et al. (1990) e HE e QIN (1993) e, a análise não-linear física de placas, em trabalhos de KARAM e TELLES (1992), RIBEIRO (1992). 
As hipóteses de KIRCHHOFF (1850), também chamadas de Teoria Clássica de Kirchhof, partindo-se da formulação direta, é a mais utilizada para análise de placas. Considerando estas hipóteses, PAIVA (1987) sugeriu diversas alternativas para o equacionamento do problema de placas, adotando-se para tanto, de equações integrais para deslocamento transversal e sua primeira derivada na direção normal do contorno, ou de duas equações integrais para o deslocamento transversal em dois pontos distintos, sendo um destes pontos situados sobre o contorno e o outro fora dele.

HARTLEY et al. (1988) (1989) trabalharam os problemas de singularidades que aparecem nos integrandos e a determinação de valores nos pontos internos, desenvolvendo um modelo de integração analítica para evitar os problemas de instabilidade numérica. KATSIKADELIS \& ARMENAKAS (1989) adotaram a combinação do Método dos Elementos de Contorno com o Método das Diferenças Finitas para a solução de duas equações integrais e duas equações diferenciais e, CAMP \& GIPSON (1990) utilizaram diversos tipos de elementos de contorno isoparamétricos, sendo as integrais, calculadas analiticamente.

Quanto à análise dinâmica em placas, o trabalho de VIVOLI e FILIPPI (1974) foi um dos precursores, utilizando o método indireto sobre soluções fundamentais derivadas das funções de Hankel e obtendo coeficientes da matriz de autovalores em função da freqüência. Posteriormente, BEZINE (1980) propôs uma formulação mista, envolvendo contorno e domínio para a análise de vibrações. NIWA et al. (1982) também apresentaram estudos utilizando o método indireto, enquanto WONG e HUTCHINSON (1981) utilizaram o método direto. Na sequência, demais trabalhos como NARDINI e BREBBIA (1982), NIWA et al. (1981) (1982), KITAHARA (1985), COSTA JR. (1985)(1988), TANAKA et al. (1987), KATSIKADELIS (1989), PROVIDAKIS e BESKOS (1989) e WEISS e MOSHAIOV (1993) também contribuíram de forma importante para a análise modal de vibração de placas.

A análise transiente foi observada no trabalho de CRUSE e RIZZO(1968), para problemas de elasto-dinâmica geral e, o de BEZINE e GAMBY (1982), que usou de soluções fundamentais de dinâmica de placas esbeltas, em função do tempo.

Placas sobre fundação elástica foi o objetivo de análise nos trabalhos iniciados por KATSIKADELIS e ARMENAKAS (1984), COSTA e BREBBIA (1985), 
SILVA e VENTURINI (1988), TEJERINA CALDERÓN e VENTURINI (1997), PAIVA e BUTTERFIELD (1997), PAIVA e TRONDI (1996) e PAIVA e MENDONÇA (1997).

A consideração da não-linearidade geométrica para solução de problemas de placas iniciou-se com KAMIYA e SAWAKY (1982) e SAVAKI et al. (1989) (1990). Já a consideração da não-linearidade física do material iniciou-se com MOSHAIOV e VORUS (1986), seguindo PARIS e LEÓN (1987) analisando placas e a variação de temperatura ao longo da sua espessura e, CHUEIRI (1994) com análise de placas esbeltas. Mais recentemente, estudos de WAIDEMAM e VENTURINI (2009) consideraram o método dos elementos de contorno para placas enrijecidas considerando-se não-linearidades física e geométrica.

Além da aplicação do método as placas, estendeu-se a análise para estruturas compostas de placas, vigas e pilares, usuais na engenharia de estruturas e aplicáveis aos pavimentos de edifícios. Inicialmente BEZINE (1981), considerou o problema de placas com apoios em seu domínio que poderiam ser utilizado para simular uma placa apoiada em colunas rígidas, seguido dos trabalhos de PARIS e LEÓN (1985), PAIVA (1987) (1991) e HARTLEY et al. (1992). Desde então vários autores como: PAIVA e VENTURINI (1985) (1987), NG et al. (1989), HARTLEY e ABDEL-AKHER (1993), HU e HARTLEY (1994), PAIVA e OLIVEIRA NETO (1995), PAIVA (1996), HARTLEY (1996), desenvolveram formulações para a análise de pavimentos (Figura 1.2) via Método dos Elementos de Contorno (M.E.C.),.
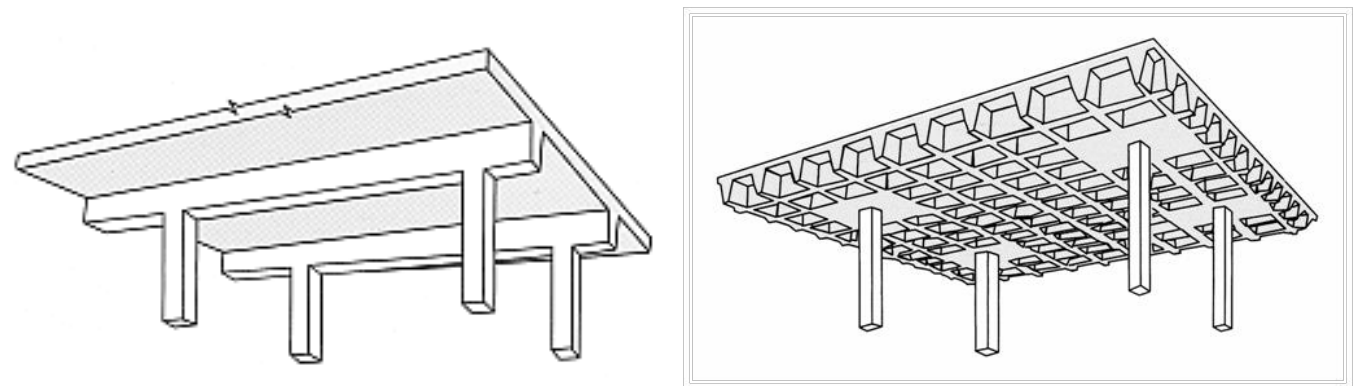

Figura 1.2 - Modelo placa-viga de um pavimento de edifício.

Nesta linha de aplicação para pavimento de edifícios, OLIVEIRA NETO (1998) apresentou uma formulação com três parâmetros nodais de deslocamentos para sua representação integral: deslocamento transversal e suas derivadas nas direções normal e tangencial ao contorno e, dois valores nodais para os esforços: momento normal e força cortante equivalente. Com esta formulação são obtidas três equações integrais de contorno por nó. 
Trabalhos mais recentes foram abordados por FERNANDES \& VENTURINI (2005), abordando a não linearidade. LEONETTI et al. (2009) propõem um modelo simétrico de elementos de contorno para análise de placas de Kirchhoff. SAPOUNTZAKIS \& DIKAROS (2008 e 2012) abordam o método AEM (Analog Equation Method) que é baseado no método dos elementos de contorno.

Demais avanços tem sido feitos quanto à implementação de novas funções aproximadoras a fim de garantir um aperfeiçoamento numérico do método como demonstrado no trabalho de OLIVEIRA \& PAIVA (2004) e como será abordado neste trabalho. $O$ presente trabalho também aborda as considerações tomadas por MENDOÇA \& PAIVA (2008 e 2010) que consideraram a equação diferencial aplicada aos elementos de viga acoplados com os elementos de contorno na placa.

No âmbito geral, o trabalho proposto tem por objetivo complementar os estudos que vêm sendo realizados, visando o desenvolvimento da formulação dos métodos dos elementos de contorno para a análise da interação placa-viga. Estudos estes, que vêm sendo desenvolvidos desde BEZINE (1981) até MENDONÇA \& PAIVA (2008).

Neste trabalho, pretende-se juntamente com o exposto em MENDONÇA \& PAIVA (2008), apresentar uma formulação para a análise da interação placa-viga e, de forma complementar, agregar a utilização dos parâmetros nodais da viga referentes à flexão e o cálculo dos esforços nas vigas (Figura 1.3) diretamente a partir da solução da equação diferencial e dos deslocamentos obtidos para seus nós.

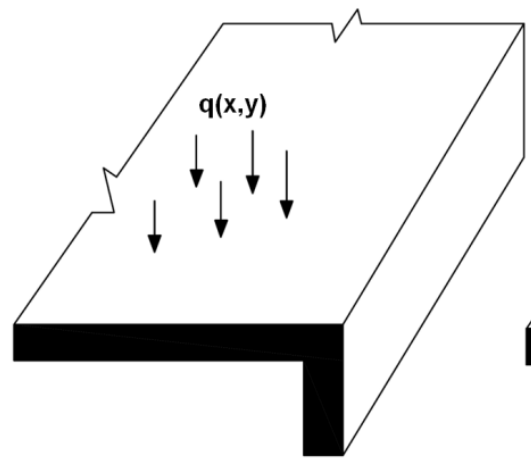

(a)

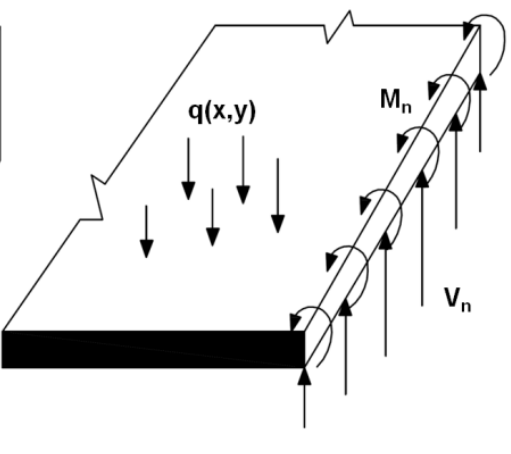

(b)

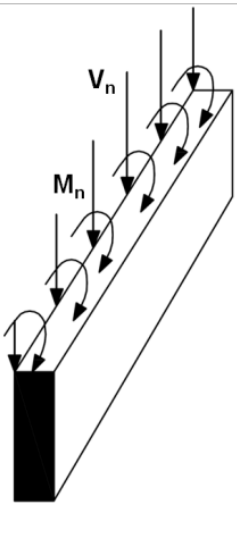

Figura 1.3 - (a) Placa com viga de canto; (b) Momento distribuído e força cortante entre a placa e a viga. Fonte: Hu e Hartley (1994) 
Este trabalho está dividido em cinco capítulos, de acordo com os seguintes objetivos:

- O capítulo 1 apresenta um breve levantamento bibliográfico sobre como vem sendo tratada a abordagem de placas via método dos elementos de contorno e uma introdução ao tema proposto;

- No capítulo 2 são apresentados um resumo da teoria de placas delgadas de Kirchhoff e a formulação teórica das expressões diferenciais das grandezas envolvidas na formulação de placas em função do deslocamento transversal w;

- No capítulo 3 são obtidas as equações integrais dos três parâmetros em deslocamento envolvidos na teoria de placas, deslocamento transversal w de suas derivadas $\partial w / \partial n$ e $\partial w / \partial s$ para pontos do contorno;

- No capítulo 4 é feita a interação dos elementos de placa com os elementos de viga;

- No capítulo 5 ocorre a avaliação numérica da formulação proposta através de diversos exemplos numéricos;

- Por fim, são apresentadas as conclusões e referências bibliográficas abordadas. 


\section{FUNDAMENTOS DA TEORIA DE PLACAS DELGADAS}

\subsection{Introdução}

Os elementos estruturais, em relação às suas dimensões, podem ser classificados como:

a) lineares, ou de barras (duas dimensões pequenas em relação à terceira);

b) de superfície, ou laminares (uma dimensão pequena em relação as outras);

c) tridimensionais, ou blocos (as três dimensões têm a mesma ordem de grandeza).

Os elementos de superfície, ou laminares (chamados também de folhas), segundo sua forma e direção de aplicação dos esforços, podem ser subdivididos em:

a) chapas (com superfície média plana e forças externas aplicadas nesse plano);

b) placas (com superfície média plana e forças externas normais a esse plano);

c) cascas (com superfície média não plana).

A superfície média, ou plano médio, é a superfície equidistante às duas superfícies que definem o elemento laminar. Dentre os elementos estruturais de superfície, a placa é um dos mais utilizados nas estruturas usuais. A placa é definida usualmente como um corpo limitado por duas superfícies planas, podendo-se admitir pequena curvatura da superfície média. A distância entre estas superfícies, chamada espessura (que pode ser variável), é pequena se comparada com as outras dimensões. Dependendo das propriedades do material que constitui a placa, esta pode ser:

a) anisotrópica (com propriedades diferentes em qualquer direção); 
b) ortótropa (com propriedades diferentes em duas direções ortogonais);

c) isótropa (com as mesmas propriedades em qualquer direção).

Dependendo da relação ( $h / a)$ entre a espessura " $h$ " e a menor dimensão "a" medida no plano da placa, ela pode ser classificada como: muito delgada, delgada e espessa. $O$ estudo dos fundamentos das placas delgadas corresponde à chamada Teoria de Kirchhoff (KIRCHHOFF, 1850), que em geral interpreta bem o comportamento das placas que tem relação (h/a) entre 1/5 a 1/100 (TIMOSHENKO \& WOINOWSKY-KRIEGER, 1959). No presente trabalho, serão consideradas apenas as placas delgadas isótropas, submetidas a carregamento transversal e ortogonal ao plano médio.

\subsection{Hipóteses}

A teoria de flexão das placas delgadas de KIRCHHOFF se baseia nas seguintes hipóteses fundamentais:

- Os pontos do plano médio da placa só se deslocam segundo a normal ao plano e os valores desses deslocamentos são pequenos em relação ao vão;

- Todos os pontos contidos em uma normal ao plano médio se mantêm numa linha reta que permanece ortogonal a deformada após a deformação;

- A tensão normal na direção perpendicular ao plano médio é nula.

A fim de conhecer o comportamento estrutural dos elementos de placas, representam-se na da Figura 2.1 os estados de tensões com o carregamento transversal distribuído $(\mathrm{g})$ atuante. Como resultante destas tensões, tem-se os momentos fletores e volvente e forças cortantes (2.1): 

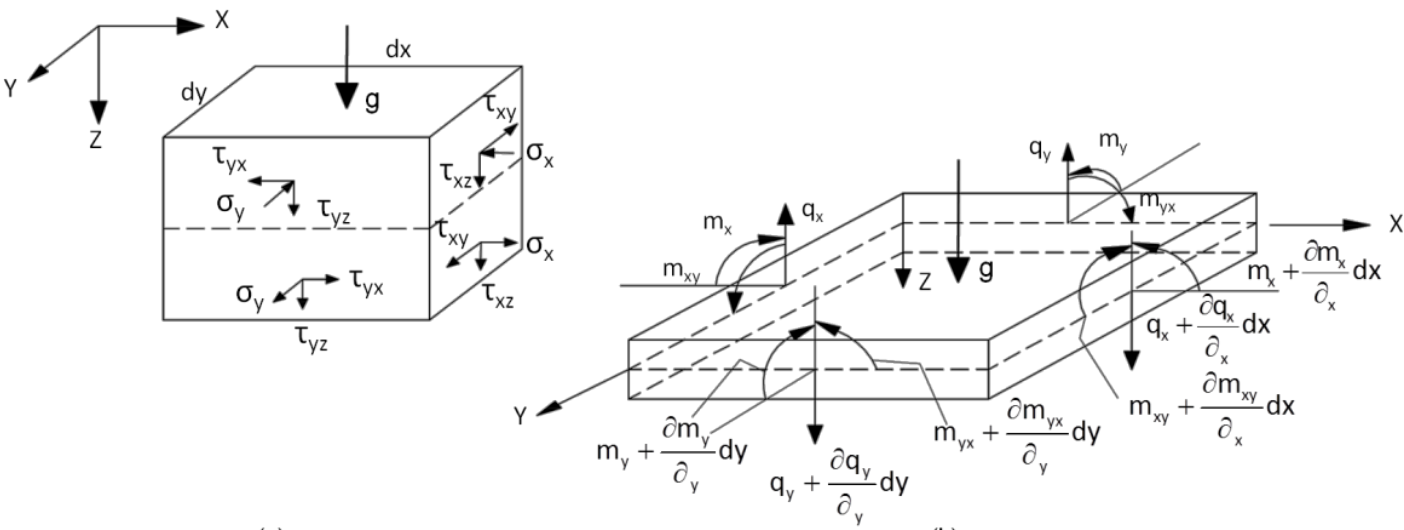

(a)

(b)

Figura 2.1- Tensões e esforços: elemento de placa.

$$
\begin{gathered}
-\frac{h}{2} \leq z \leq+\frac{h}{2} \\
m_{x}=\int_{-\frac{h}{2}}^{\frac{h}{2}} \sigma_{x} z d z \\
\mathrm{~m}_{\mathrm{y}}=\int_{-\mathrm{h} / 2}^{\mathrm{h} / 2} \sigma_{\mathrm{y}} \mathrm{zdz} \\
\mathrm{m}_{\mathrm{xy}}=\int_{-\mathrm{h} / 2}^{\mathrm{h} / 2} \tau_{\mathrm{xy}} \mathrm{zdz} \\
\mathrm{q}_{\mathrm{x}}=\int_{-\mathrm{h} / 2}^{\mathrm{h} / 2} \tau_{\mathrm{xz}} \mathrm{dz} \\
\mathrm{q}_{\mathrm{y}}=\int_{-\mathrm{h} / 2}^{\mathrm{h} / 2} \tau_{\mathrm{yz}} \mathrm{dz}
\end{gathered}
$$

Onde h é a espessura da placa.

A partir da imposição de equilíbrio de forças verticais e momentos em torno dos eixos $x$ e y, obtém-se as seguintes equações de equilíbrio:

$$
\frac{\partial q_{x}}{\partial x}+\frac{\partial q_{y}}{\partial y}+g=0
$$




$$
\begin{aligned}
& \frac{\partial \mathrm{m}_{\mathrm{x}}}{\partial \mathrm{x}}+\frac{\partial \mathrm{m}_{\mathrm{xy}}}{\partial \mathrm{y}}=\mathrm{q}_{\mathrm{x}} \\
& \frac{\partial \mathrm{m}_{\mathrm{y}}}{\partial \mathrm{y}}+\frac{\partial \mathrm{m}_{\mathrm{yx}}}{\partial \mathrm{x}}=\mathrm{q}_{\mathrm{y}}
\end{aligned}
$$

Eliminando $q_{x}$ e $q_{y}$ no sistema de equações definidas por (2.8), (2.9) e (2.10) e considerando-se a simetria do tensor dos momentos $\left(\mathrm{m}_{\mathrm{yx}}=\mathrm{m}_{\mathrm{xy}}\right)$, obtém-se:

$$
\frac{\partial^{2} m_{x}}{\partial x^{2}}+\frac{\partial^{2} m_{y}}{\partial y^{2}}+2 \frac{\partial^{2} m_{x y}}{\partial x \partial y}=-g
$$

As componentes de deformações $\varepsilon_{x}$ e $\varepsilon_{y}$ e a distorção $\gamma_{x y}$ em um ponto distante de $z$ da superfície média da placa podem ser escritas em função do deslocamento transversal w:

$$
\begin{gathered}
\varepsilon_{\mathrm{x}}=-\mathrm{z} \frac{\partial^{2} \mathrm{w}}{\partial \mathrm{x}^{2}} \\
\varepsilon_{\mathrm{y}}=-\mathrm{z} \frac{\partial^{2} \mathrm{w}}{\partial \mathrm{y}^{2}} \\
\gamma_{\mathrm{xy}}=-2 \mathrm{z} \frac{\partial^{2} \mathrm{w}}{\partial \mathrm{x} \partial \mathrm{y}}
\end{gathered}
$$

Conhecidas as deformações em um dado ponto da placa, as tensões correspondentes se determinam a partir da Lei de Hooke, conforme segue:

$$
\begin{gathered}
\sigma_{x}=\frac{E}{1-v^{2}}\left(\varepsilon_{x}+v \varepsilon_{y}\right) \\
\sigma_{y}=\frac{E}{1-v^{2}}\left(\varepsilon_{y}+v \varepsilon_{x}\right) \\
\tau_{x y}=G \gamma_{x y}
\end{gathered}
$$

Onde:

E : módulo de elasticidade longitudinal; 
$v$ : coeficiente de Poisson;

G : módulo de elasticidade transversal (2.17);

$$
\mathrm{G}=\frac{\mathrm{E}}{2(1+v)}
$$

Substituindo nas equações (2.14), (2.15) e (2.16) as expressões de deformações relativas $\varepsilon_{\mathrm{x}}$ e $\varepsilon_{\mathrm{y}}$ e a distorção $\gamma_{\mathrm{xy}},(2.11),(2.12)$ e (2.13), obtêm-se:

$$
\begin{gathered}
\sigma_{\mathrm{x}}=\frac{-\mathrm{E}}{1-v^{2}}\left(\frac{\partial^{2} \mathrm{w}}{\partial \mathrm{x}^{2}}+v \frac{\partial^{2} \mathrm{w}}{\partial \mathrm{y}^{2}}\right) \mathrm{z} \\
\sigma_{\mathrm{y}}=\frac{-\mathrm{E}}{1-v^{2}}\left(\frac{\partial^{2} \mathrm{w}}{\partial \mathrm{y}^{2}}+v \frac{\partial^{2} \mathrm{w}}{\partial \mathrm{x}^{2}}\right) \mathrm{z} \\
\tau_{\mathrm{xy}}=-2 \mathrm{G} \frac{\partial^{2} \mathrm{w}}{\partial \mathrm{x} \partial \mathrm{y}} \mathrm{z}
\end{gathered}
$$

Substituindo nas expressões (2.2), (2.3) e (2.4) os valores das componentes de tensão $\sigma_{\mathrm{x}}, \sigma_{\mathrm{y}}$ e $\tau_{\mathrm{xy}},(2.18),(2.19)$ e (2.20), obtêm-se:

$$
\begin{gathered}
\mathrm{m}_{\mathrm{x}}=-\mathrm{D}\left(\frac{\partial^{2} \mathrm{w}}{\partial \mathrm{x}^{2}}+v \frac{\partial^{2} \mathrm{w}}{\partial \mathrm{y}^{2}}\right) \\
\mathrm{m}_{\mathrm{y}}=-\mathrm{D}\left(\frac{\partial^{2} \mathrm{w}}{\partial \mathrm{y}^{2}}+v \frac{\partial^{2} \mathrm{w}}{\partial \mathrm{x}^{2}}\right) \\
\mathrm{m}_{\mathrm{xy}}=-\mathrm{D}(1-v) \frac{\partial^{2} \mathrm{w}}{\partial \mathrm{x} \partial \mathrm{y}}
\end{gathered}
$$

Onde:

$\mathrm{D}$ : rigidez à flexão, é dado por:

$$
\mathrm{D}=\frac{\mathrm{E} \cdot \mathrm{h}^{3}}{12\left(1+v^{2}\right)}
$$

Substituindo em (2.10), $\mathrm{m}_{\mathrm{x}}, \mathrm{m}_{\mathrm{y}}$ e $\mathrm{m}_{\mathrm{xy}}$, por suas expressões, dadas por (2.21), (2.22) e (2.23), obtém-se a conhecida equação diferencial de placas: 


$$
\frac{\partial^{4} w}{\partial x^{4}}+2 \frac{\partial^{4} w}{\partial x^{2} \partial y^{2}}+\frac{\partial^{4} w}{\partial y^{4}}=\frac{g}{D}
$$

Utilizando o operador de Laplace (2.26) na equação (2.10):

$$
\nabla^{2}=\frac{\partial^{2}}{\partial x^{2}}+\frac{\partial^{2}}{\partial y^{2}}
$$

A equação diferencial de placas (2.25) pode ser reescrita na seguinte forma:

$$
\nabla^{2} \nabla^{2} w=\left(\frac{\partial^{2}}{\partial x^{2}}+\frac{\partial^{2}}{\partial y^{2}}\right)\left(\frac{\partial^{2} w}{\partial x^{2}}+\frac{\partial^{2} w}{\partial y^{2}}\right)=\frac{g}{D}
$$

e ainda:

$$
\begin{aligned}
& q_{x}=-D \frac{\partial}{\partial x}\left(\frac{\partial^{2} w}{\partial x^{2}}+\frac{\partial^{2} w}{\partial y^{2}}\right) \\
& q_{y}=-D \frac{\partial}{\partial y}\left(\frac{\partial^{2} w}{\partial x^{2}}+\frac{\partial^{2} w}{\partial y^{2}}\right)
\end{aligned}
$$

Ou ainda:

$$
\begin{aligned}
& \mathrm{q}_{\mathrm{x}}=-\mathrm{D} \frac{\partial}{\partial \mathrm{x}}\left(\nabla^{2} \mathrm{w}\right) \\
& \mathrm{q}_{\mathrm{y}}=-\mathrm{D} \frac{\partial}{\partial \mathrm{y}}\left(\nabla^{2} \mathrm{w}\right)
\end{aligned}
$$

As componentes de tensões e esforços, podem agora, ser escritas para um sistema genérico de coordenadas $(n, s)$, onde o eixo $n$ forma um ângulo $\alpha$ com o semi-eixo positivo $\mathrm{x}$.

Tomando como base o elemento de placa 1-2-3, representado na Figura 2.2: 




Figura 2.2 - Componentes de tensão no elemento de placa 1-2-3.

Escrevem-se as relações das tensões e dos esforços referentes ao lado 3-2 nas direções n e s:

$$
\begin{gathered}
\sigma_{\mathrm{n}}=\sigma_{\mathrm{x}} \cos \alpha+\sigma_{\mathrm{y}} \operatorname{sen} \alpha+2 \tau_{\mathrm{xy}} \operatorname{sen} \alpha \cos \alpha \\
\tau_{\mathrm{ns}}=\left(\sigma_{\mathrm{y}}-\sigma_{\mathrm{x}}\right) \operatorname{sen} \alpha \cos \alpha+\tau_{\mathrm{xy}}\left(\cos ^{2} \alpha-\operatorname{sen}^{2} \alpha\right) \\
\mathrm{m}_{\mathrm{n}}=\mathrm{m}_{\mathrm{x}} \cos ^{2} \alpha+\mathrm{m}_{\mathrm{y}} \operatorname{sen}^{2} \alpha+2 \mathrm{~m}_{\mathrm{xy}} \operatorname{sen} \alpha \cos \alpha \\
\mathrm{m}_{\mathrm{ns}}=\left(\mathrm{m}_{\mathrm{y}}-\mathrm{m}_{\mathrm{x}}\right) \operatorname{sen} \alpha \cos \alpha+\mathrm{m}_{\mathrm{xy}}\left(\cos ^{2} \alpha-\operatorname{sen}^{2} \alpha\right)
\end{gathered}
$$

Da condição de equilíbrio de forças verticais sobre o elemento, tem-se:

$$
\mathrm{q}_{\mathrm{n}} \mathrm{ds}=\mathrm{q}_{\mathrm{x}} \mathrm{ds} \cos \alpha+\mathrm{q}_{\mathrm{y}} \mathrm{ds} \operatorname{sen} \alpha
$$

$\mathrm{Ou}$

$$
\mathrm{q}_{\mathrm{n}}=\mathrm{q}_{\mathrm{x}} \cos \alpha+\mathrm{q}_{\mathrm{y}} \operatorname{sen} \alpha
$$

Para se encontrar a solução da equação diferencial de placas é necessário impor condições de contorno relacionadas ao deslocamento w, ao giro $\partial \mathrm{w} / \partial \mathrm{m}$ e aos esforços (momento fletor, momento volvente e força cortante, por unidade de comprimento). KIRCHHOFF (1850), entretanto, demonstrou que é possível escrever as condições de contorno relativas à força cortante e ao momento volvente numa única condição. Para entender esta proposição, considera-se um 
ponto genérico, $\mathrm{P}$, na borda da placa e dois elementos de comprimento ds, adjacentes a este ponto, conforme mostra a Figura 2.3.


(b)

(c)

Figura 2.3 - Momento volvente no contorno.

O momento volvente resultante em um elemento de comprimento ds do contorno tem valores mnsds e $\left(\mathrm{mns}_{\mathrm{ns}}+\partial \mathrm{mns} / \partial \mathrm{s}\right) \mathrm{ds}$. Sendo possível interpretar estes momentos como resultantes de binários de forças atuando agora nas laterais dos elementos de contorno, surgindo uma resultante final no ponto $\mathrm{P}$ de valor $(\partial \mathrm{mns} / \partial \mathrm{s})$ ds. Esta força, somada com a força cortante, qnds, resulta em um esforço denominado força cortante equivalente, por unidade de comprimento, e vale:

$$
\mathrm{V}_{\mathrm{n}}=\mathrm{q}_{\mathrm{n}}+\frac{\partial \mathrm{m}_{\mathrm{ns}}}{\partial \mathrm{s}}
$$

Para o caso de $\mathrm{n}$ coincidir com $\mathrm{x}$ ou $\mathrm{y}$, tem-se:

$$
\begin{aligned}
& \mathrm{V}_{\mathrm{x}}=\mathrm{q}_{\mathrm{x}}+\frac{\partial \mathrm{m}_{\mathrm{xy}}}{\partial \mathrm{y}} \\
& \mathrm{V}_{\mathrm{y}}=\mathrm{q}_{\mathrm{y}}+\frac{\partial \mathrm{m}_{\mathrm{xy}}}{\partial \mathrm{x}}
\end{aligned}
$$

\subsection{Equações de placas em coordenadas polares}

Para alguns problemas de análise estrutural o uso de coordenadas polares nas equações diferenciais se torna mais fácil de analisar. Assim, um ponto $P$ de coordenadas $(x, y)$ pode ser definido em função de $r$ e $\theta$, que são 
respectivamente, a distância deste ponto à origem do sistema de coordenadas $(\mathrm{x}, \mathrm{y})$ e o ângulo entre o segmento OP e o eixo Ox (conforme Figura 2.4).

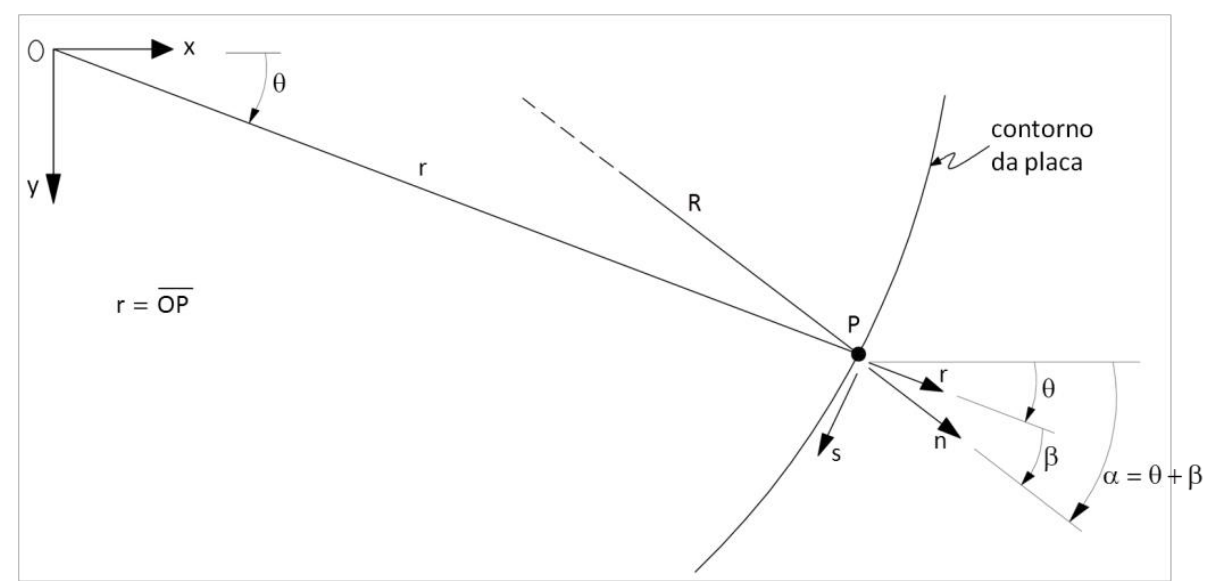

Figura 2.4 - Sistemas de coordenadas cartesianas e polares para o contorno da placa.

Assim as relações entre os dois sistemas ficam determinadas por:

$$
\begin{aligned}
& x=r \cdot \cos \theta \\
& y=r \cdot \operatorname{sen} \theta
\end{aligned}
$$

Ou ainda:

$$
\begin{aligned}
& r^{2}=x^{2}+y^{2} \\
& \theta=\operatorname{arctag} \frac{y}{x}
\end{aligned}
$$

Usando dessas relações, encontram-se suas derivadas:

$$
\begin{gathered}
\frac{\partial r}{\partial x}=\frac{x}{r}=\cos \theta \\
\frac{\partial r}{\partial y}=\frac{y}{r}=\operatorname{sen} \theta \\
\frac{\partial \theta}{\partial x}=\frac{-y}{r^{2}}=\frac{-\operatorname{sen} \theta}{r} \\
\frac{\partial \theta}{\partial y}=\frac{x}{r^{2}}=\frac{\cos \theta}{r}
\end{gathered}
$$


Escrevendo as derivadas do deslocamento w da placa, de coordenadas cartesianas para coordenadas polares, através de:

Na direção x:

$$
\frac{\partial \mathrm{w}}{\partial \mathrm{x}}=\frac{\partial \mathrm{w}}{\partial \mathrm{r}} \cdot \frac{\partial \mathrm{r}}{\partial \mathrm{x}}+\frac{\partial \mathrm{w}}{\partial \theta} \cdot \frac{\partial \theta}{\partial \mathrm{x}}
$$

Ou ainda,

$$
\frac{\partial \mathrm{w}}{\partial \mathrm{x}}=\frac{\partial \mathrm{w}}{\partial \mathrm{r}} \cdot \cos \theta+\frac{\partial \mathrm{w}}{\partial \theta} \cdot\left(\frac{-\operatorname{sen} \theta}{\mathrm{r}}\right)
$$

Na direção y:

$$
\frac{\partial \mathrm{w}}{\partial \mathrm{y}}=\frac{\partial \mathrm{w}}{\partial \mathrm{r}} \cdot \operatorname{sen} \theta+\frac{\partial \mathrm{w}}{\partial \theta} \cdot\left(\frac{\cos \theta}{\mathrm{r}}\right)
$$

Resultando as derivadas de segunda ordem em:

$$
\begin{aligned}
& \begin{aligned}
\frac{\partial^{2} \mathrm{w}}{\partial \mathrm{x}^{2}}=\frac{\partial}{\partial \mathrm{x}}\left(\frac{\partial \mathrm{w}}{\partial \mathrm{r}}\right. & \left.\cos \theta-\frac{\partial \mathrm{w}}{\partial \theta} \frac{\operatorname{sen} \theta}{\mathrm{r}}\right) \\
= & \frac{\partial^{2} \mathrm{w}}{\partial \mathrm{r}^{2}} \cos ^{2} \theta+\operatorname{sen}^{2} \theta\left(\frac{1}{\mathrm{r}} \frac{\partial \mathrm{w}}{\partial \mathrm{r}}+\frac{1}{\mathrm{r}^{2}} \frac{\partial^{2} \mathrm{w}}{\partial \theta^{2}}\right)-2 \operatorname{sen} \theta \cos \theta \frac{\partial}{\partial \mathrm{r}}\left(\frac{1}{\mathrm{r}} \frac{\partial \mathrm{w}}{\partial \theta}\right)
\end{aligned} \\
& \begin{aligned}
\frac{\partial^{2} \mathrm{w}}{\partial \mathrm{y}^{2}}=\frac{\partial}{\partial \mathrm{y}}\left(\frac{\partial \mathrm{w}}{\partial \mathrm{r}}\right. & \left.\operatorname{sen} \theta+\frac{\partial \mathrm{w}}{\partial \theta} \frac{\cos \theta}{\mathrm{r}}\right) \\
& =\frac{\partial^{2} \mathrm{w}}{\partial \mathrm{r}^{2}} \operatorname{sen}^{2} \theta+\cos ^{2} \theta\left(\frac{1}{\mathrm{r}} \frac{\partial \mathrm{w}}{\partial \mathrm{r}}+\frac{1}{\mathrm{r}^{2}} \frac{\partial^{2} \mathrm{w}}{\partial \theta^{2}}\right)+2 \operatorname{sen} \theta \cos \theta \frac{\partial}{\partial \mathrm{r}}\left(\frac{1}{\mathrm{r}} \frac{\partial \mathrm{w}}{\partial \theta}\right)
\end{aligned}
\end{aligned}
$$

e

$$
\begin{aligned}
\frac{\partial^{2} w}{\partial x \partial y}=\frac{\partial}{\partial y}\left(\frac{\partial w}{\partial r}\right. & \left.\cos \theta-\frac{\partial w}{\partial \theta} \frac{\operatorname{sen} \theta}{r}\right) \\
= & -\operatorname{sen} \theta \cos \theta\left(\frac{1}{r} \frac{\partial w}{\partial r}+\frac{1}{r^{2}} \frac{\partial^{2} w}{\partial \theta^{2}}-\frac{\partial^{2} w}{\partial r^{2}}\right)+\left(\cos ^{2} \theta-\operatorname{sen}^{2} \theta\right) \frac{\partial}{\partial r}\left(\frac{1}{r} \frac{\partial w}{\partial \theta}\right)
\end{aligned}
$$

Somando as expressões (2.52) e (2.53), chega-se ao operador diferencial de Laplace expresso em coordenadas polares: 


$$
\left(\frac{\partial^{2}}{\partial \mathrm{x}^{2}}+\frac{\partial^{2}}{\partial \mathrm{y}^{2}}\right) \mathrm{w}=\left(\frac{\partial^{2}}{\partial \mathrm{r}^{2}}+\frac{1}{\mathrm{r}} \frac{\partial}{\partial \mathrm{r}}+\frac{1}{\mathrm{r}^{2}} \frac{\partial^{2}}{\partial \theta^{2}}\right) \mathrm{w}
$$

ficando, portanto, a equação diferencial da placa (2.27), em coordenadas polares, dada por:

$$
\left(\frac{\partial^{2}}{\partial \mathrm{x}^{2}}+\frac{\partial^{2}}{\partial \mathrm{y}^{2}}\right)\left(\frac{\partial^{2} \mathrm{w}}{\partial \mathrm{x}^{2}}+\frac{\partial^{2} \mathrm{w}}{\partial \mathrm{y}^{2}}\right)=\left(\frac{\partial^{2}}{\partial \mathrm{r}^{2}}+\frac{1}{\mathrm{r}} \frac{\partial}{\partial \mathrm{r}}+\frac{1}{\mathrm{r}^{2}} \frac{\partial^{2}}{\partial \theta^{2}}\right)\left(\frac{\partial^{2} \mathrm{w}}{\partial \mathrm{r}^{2}}+\frac{1}{\mathrm{r}} \frac{\partial \mathrm{w}}{\partial \mathrm{r}}+\frac{1}{\mathrm{r}^{2}} \frac{\partial^{2} \mathrm{w}}{\partial \theta^{2}}\right)=\frac{\mathrm{g}}{\mathrm{D}}
$$

Substituindo as equações (2.52), (2.53) e (2.54) nas expressões(2.21), (2.22), (2.23), (2.28) e (2.29), têm-se:

$$
\begin{gathered}
\mathrm{m}_{\mathrm{x}}=-\mathrm{D}\left[\frac{\partial^{2} \mathrm{w}}{\partial \mathrm{r}^{2}}\left(\cos ^{2} \theta+v \cdot \operatorname{sen}^{2} \theta\right)+\left(\frac{1}{\mathrm{r}} \frac{\partial \mathrm{w}}{\partial \mathrm{r}}+\frac{1}{\mathrm{r}^{2}} \frac{\partial^{2} \mathrm{w}}{\partial \theta^{2}}\right)\left(\operatorname{sen}^{2} \theta+v \cdot \cos ^{2} \theta\right)\right. \\
\left.-2(1-v) \operatorname{sen} \theta \cos \theta \frac{\partial}{\partial \mathrm{r}}\left(\frac{1}{\mathrm{r}} \frac{\partial \mathrm{w}}{\partial \theta}\right)\right] \\
\mathrm{m}_{\mathrm{y}}=-\mathrm{D}\left[\frac{\partial^{2} \mathrm{w}}{\partial \mathrm{r}^{2}}\left(\operatorname{sen}^{2} \theta+v \cdot \cos ^{2} \theta\right)+\left(\frac{1}{\mathrm{r}} \frac{\partial \mathrm{w}}{\partial \mathrm{r}}+\frac{1}{\mathrm{r}^{2}} \frac{\partial^{2} \mathrm{w}}{\partial \theta^{2}}\right)\left(\cos ^{2} \theta+v \cdot \operatorname{sen}^{2} \theta\right)\right. \\
\mathrm{m}_{\mathrm{xy}}=-\mathrm{D}(1-v)\left[\left(\frac{\partial^{2} \mathrm{w}}{\partial \mathrm{r}^{2}}-\frac{1}{\mathrm{r}} \frac{\partial \mathrm{w}}{\partial \mathrm{r}}-\frac{1}{\mathrm{r}^{2}} \frac{\partial^{2} \mathrm{w}}{\partial \theta^{2}}\right) \operatorname{sen} \theta \cos \theta+\left(\cos ^{2} \theta-\operatorname{sen}^{2} \theta\right) \frac{\partial}{\partial \mathrm{r}}\left(\frac{1}{\mathrm{r}} \frac{\partial \mathrm{w}}{\partial \theta}\right)\right] \\
\mathrm{q}_{\mathrm{x}}=-\mathrm{D}\left[\cos \theta \frac{\partial}{\partial \mathrm{r}}\left(\frac{\partial^{2} \mathrm{w}}{\partial \mathrm{r}^{2}}+\frac{1}{\mathrm{r}} \frac{\partial \mathrm{w}}{\partial \mathrm{r}}+\frac{1}{\mathrm{r}^{2}} \frac{\partial^{2} \mathrm{w}}{\partial \theta^{2}}\right)-\frac{\operatorname{sen} \theta}{\mathrm{r}} \frac{\partial}{\partial \theta}\left(\frac{\partial^{2} \mathrm{w}}{\partial \mathrm{r}^{2}}+\frac{1}{\mathrm{r}} \frac{\partial \mathrm{w}}{\partial \mathrm{r}}+\frac{1}{\mathrm{r}^{2}} \frac{\partial^{2} \mathrm{w}}{\partial \theta^{2}}\right)\right] \\
\mathrm{q}_{\mathrm{y}}=-\mathrm{D}\left[\operatorname{sen} \theta \frac{\partial}{\partial \mathrm{r}}\left(\frac{\partial^{2} \mathrm{w}}{\partial \mathrm{r}^{2}}+\frac{1}{\mathrm{r}} \frac{\partial \mathrm{w}}{\partial \mathrm{r}}+\frac{1}{\mathrm{r}^{2}} \frac{\partial^{2} \mathrm{w}}{\partial \theta^{2}}\right)+\frac{\cos \theta}{\mathrm{r}} \frac{\partial}{\partial \theta}\left(\frac{\partial^{2} \mathrm{w}}{\partial \mathrm{r}^{2}}+\frac{1}{\mathrm{r}} \frac{\partial \mathrm{w}}{\partial \mathrm{r}}+\frac{1}{\mathrm{r}^{2}} \frac{\partial^{2} \mathrm{w}}{\partial \theta^{2}}\right)\right]
\end{gathered}
$$

Conhecidas as expressões, em coordenadas polares, de $\mathrm{m}_{\mathrm{x}}, \mathrm{m}_{\mathrm{y}}, \mathrm{m}_{\mathrm{xy}}$, $\mathrm{q}_{\mathrm{x}}$ e $\mathrm{q}_{\mathrm{y}}$ podem ser deduzidas, nesse mesmo sistema de coordenadas, as expressões de $m_{n}, m_{n s}$ e $V_{n}$ em um ponto $P$ genérico do contorno, onde $n$ e $s$ são vetores de origem em $\mathrm{P}$ e normal e tangente ao contorno (conforme Figura 2.4).

Substituindo $\mathrm{m}_{\mathrm{x}}, \mathrm{m}_{\mathrm{y}}$ e $\mathrm{m}_{\mathrm{xy}}$ das expressões (2.57), (2.58) e (2.59), bem como $\alpha=\theta+\beta$ da Figura 2.4 nas equações (2.34) e (2.35), obtêm-se: 


$$
\begin{gathered}
\mathrm{m}_{\mathrm{n}}=-\mathrm{D}\left[\frac{\partial^{2} \mathrm{w}}{\partial \mathrm{r}^{2}}\left(\cos ^{2} \beta+v \cdot \operatorname{sen}^{2} \beta\right)+\left(\frac{1}{\mathrm{r}} \frac{\partial \mathrm{w}}{\partial \mathrm{r}}+\frac{1}{\mathrm{r}^{2}} \cdot \frac{\partial^{2} \mathrm{w}}{\partial \theta^{2}}\right)\left(\operatorname{sen}^{2} \beta+v \cdot \cos ^{2} \beta\right)\right. \\
\left.+2(1-v) \operatorname{sen} \beta \cos \beta \frac{\partial}{\partial \mathrm{r}}\left(\frac{1}{\mathrm{r}} \frac{\partial \mathrm{w}}{\partial \theta}\right)\right] \\
\mathrm{m}_{\mathrm{s}}=-\mathrm{D}\left[\frac{\partial^{2} \mathrm{w}}{\partial \mathrm{r}^{2}}\left(\operatorname{sen}^{2} \beta+v \cdot \cos ^{2} \beta\right)+\left(\frac{1}{\mathrm{r}} \frac{\partial \mathrm{w}}{\partial \mathrm{r}}+\frac{1}{\mathrm{r}^{2}} \cdot \frac{\partial^{2} \mathrm{w}}{\partial \theta^{2}}\right)\left(\cos ^{2} \beta+v \cdot \operatorname{sen}^{2} \beta\right)\right. \\
\left.-2(1-v) \operatorname{sen} \beta \cos \beta \frac{\partial}{\partial \mathrm{r}}\left(\frac{1}{\mathrm{r}} \frac{\partial \mathrm{w}}{\partial \theta}\right)\right] \\
\mathrm{m}_{\mathrm{ns}}=-\mathrm{D}(1-v)\left[\left(\frac{\partial^{2} \mathrm{w}}{\partial \mathrm{r}^{2}}-\frac{1}{\mathrm{r}} \frac{\partial \mathrm{w}}{\partial \mathrm{r}}-\frac{1}{\mathrm{r}^{2}} \frac{\partial^{2} \mathrm{w}}{\partial \theta^{2}}\right) \operatorname{sen} \beta \cos \beta+\frac{\partial}{\partial \mathrm{r}}\left(\frac{1}{\mathrm{r}} \frac{\partial \mathrm{w}}{\partial \theta}\right)\left(\cos ^{2} \beta-\operatorname{sen}^{2} \beta\right)\right]
\end{gathered}
$$

Para se chegar à expressão da força cortante equivalente $V_{n}$, em coordenadas polares, deriva-se a parcela de momento volvente, $\mathrm{m}_{\mathrm{ns}}$, dado pela equação (2.38) em relação a s. Para a cortante tem-se:

$$
q_{n}=q_{x} \cos (\theta+\beta)+q_{y} \operatorname{sen}(\theta+\beta)
$$

Substituindo qx e qy pelas expressões (2.60) e (2.61), resulta:

$$
\mathrm{q}_{\mathrm{n}}=-\mathrm{D}\left[\cos \beta \frac{\partial}{\partial \mathrm{r}}\left(\frac{\partial^{2} \mathrm{w}}{\partial \mathrm{r}^{2}}+\frac{1}{\mathrm{r}} \frac{\partial \mathrm{w}}{\partial \mathrm{r}}+\frac{1}{\mathrm{r}^{2}} \frac{\partial^{2} \mathrm{w}}{\partial \theta^{2}}\right)+\frac{\operatorname{sen} \beta}{\mathrm{r}} \frac{\partial}{\partial \theta}\left(\frac{\partial^{2} \mathrm{w}}{\partial \mathrm{r}^{2}}+\frac{1}{\mathrm{r}} \frac{\partial \mathrm{w}}{\partial \mathrm{r}}+\frac{1}{\mathrm{r}^{2}} \frac{\partial^{2} \mathrm{w}}{\partial \theta^{2}}\right)\right]
$$

E a derivada da parcela de momento volvente, $\mathrm{mns}$ :

$$
\frac{\partial \mathrm{m}_{\mathrm{ns}}}{\partial \mathrm{s}}=\frac{\partial \mathrm{m}_{\mathrm{ns}}}{\partial \mathrm{r}} \frac{\partial \mathrm{r}}{\partial \mathrm{s}}+\frac{\partial \mathrm{m}_{\mathrm{ns}}}{\partial \theta} \frac{\partial \theta}{\partial \mathrm{s}}+\frac{\partial \mathrm{m}_{\mathrm{ns}}}{\partial \beta} \frac{\partial \beta}{\partial \mathrm{s}}
$$

Das relações abaixo:

$$
\begin{gathered}
\frac{\partial \mathrm{r}}{\partial \mathrm{s}}=\frac{\partial \mathrm{r}}{\partial \mathrm{x}} \frac{\partial \mathrm{x}}{\partial \mathrm{s}}+\frac{\partial \mathrm{r}}{\partial \mathrm{y}} \frac{\partial \mathrm{y}}{\partial \mathrm{s}}=\cos \theta(-\operatorname{sen} \alpha)+\operatorname{sen} \theta \cos \alpha \\
\alpha=\theta+\beta \quad \text { ou } \quad \beta=\alpha-\theta \\
\frac{\partial \mathrm{r}}{\partial \mathrm{s}}=-\operatorname{sen} \beta \\
\frac{\partial \theta}{\partial \mathrm{s}}=-\frac{-\operatorname{sen} \theta}{\mathrm{r}}(-\operatorname{sen} \alpha)+\frac{\cos \theta}{\mathrm{r}} \cos \alpha=\frac{\cos \beta}{\mathrm{r}}
\end{gathered}
$$


e, considerando $\mathrm{R}$ como o raio de curvatura do contorno no ponto $\mathrm{P}$,

$$
\frac{\partial \beta}{\partial \mathrm{s}}=\frac{1}{\mathrm{R}}-\frac{\cos \beta}{\mathrm{r}}
$$

e , substituindo as equações (2.70), (2.71) e (2.72) na equação (2.67), obtém-se:

$$
\frac{\partial \mathrm{m}_{\mathrm{ns}}}{\partial \mathrm{s}}=\frac{\partial \mathrm{m}_{\mathrm{ns}}}{\partial \mathrm{r}}(-\operatorname{sen} \beta)+\frac{\partial \mathrm{m}_{\mathrm{ns}}}{\partial \theta}\left(\frac{\cos \beta}{\mathrm{r}}\right)+\frac{\partial \mathrm{m}_{\mathrm{ns}}}{\partial \beta}\left(\frac{1}{\mathrm{R}}-\frac{\cos \beta}{\mathrm{r}}\right)
$$

Voltando na expressão da força cortante equivalente (2.38) e substituindo os valores encontrados para $\mathrm{q}_{\mathrm{n}}$ e para $\partial \mathrm{m}_{\mathrm{ns}} / \partial \mathrm{s}$ em coordenadas polares, (2.66) e (2.73), obtém-se finalmente:

$$
\begin{aligned}
\mathrm{V}_{\mathrm{n}}=\left[-\mathrm{D} \frac{\partial}{\partial \mathrm{r}}\left(\frac{\partial^{2} \mathrm{w}}{\partial \mathrm{r}^{2}}+\frac{1}{\mathrm{r}} \frac{\partial \mathrm{w}}{\partial \mathrm{r}}+\frac{1}{\mathrm{r}^{2}} \frac{\partial^{2} \mathrm{w}}{\partial \theta^{2}}\right)+\frac{1}{\mathrm{r}} \frac{\partial \mathrm{m}_{\mathrm{ns}}}{\partial \theta}\right] \cos \beta \\
-\left[\frac{\mathrm{D}}{\mathrm{r}} \frac{\partial}{\partial \theta}\left(\frac{\partial^{2} \mathrm{w}}{\partial \mathrm{r}^{2}}+\frac{1}{\mathrm{r}} \frac{\partial \mathrm{w}}{\partial \mathrm{r}}+\frac{1}{\mathrm{r}^{2}} \frac{\partial^{2} \mathrm{w}}{\partial \theta^{2}}\right)+\frac{\partial \mathrm{m}_{\mathrm{ns}}}{\partial \mathrm{r}}\right] \operatorname{sen} \beta+\left(\frac{1}{\mathrm{R}}-\frac{\cos \beta}{\mathrm{r}}\right) \frac{\partial \mathrm{m}_{\mathrm{ns}}}{\partial \beta}
\end{aligned}
$$

\subsection{Soluções fundamentais de placas:}

A solução fundamental de placas corresponde ao deslocamento $\mathrm{w}^{*}$ de um ponto genérico $\mathrm{q}$ de coordenadas $[\mathrm{x}(\mathrm{q}), \mathrm{y}(\mathrm{q})]$ no domínio fundamental da placa, geralmente infinito, devida a uma carga unitária aplicada em um ponto s, ponto de carregamento, de coordenadas $[\mathrm{x}(\mathrm{s}), \mathrm{y}(\mathrm{s})]$ deste mesmo domínio. É obtida, substituindo-se o carregamento transversal distribuído atuante (g) pela distribuição delta de Dirac em (2.27), isto é:

$$
\nabla^{2} \nabla^{2} \mathrm{w}^{*}=\frac{\delta(\mathrm{s}, \mathrm{q})}{\mathrm{D}}
$$

onde, $\delta(s, q)$ é a distribuição delta de Dirac.

Essa distribuição apresenta as seguintes propriedades: 


$$
\begin{array}{ll}
\mathrm{g}=\delta(\mathrm{s}, \mathrm{q})=0 & \mathrm{q} \neq \mathrm{s} \\
\mathrm{g}=\delta(\mathrm{s}, \mathrm{q})=\infty & \mathrm{q}=\mathrm{s}
\end{array}
$$

e

$$
\int \delta(\mathrm{s}, \mathrm{q}) \mathrm{d} \Omega_{\infty}=1
$$

Ou seja, a resultante do carregamento transversal aplicado sobre o domínio fundamental é uma força unitária aplicada no ponto s.

Sendo $\Phi(q)$ uma função contínua qualquer, definida no domínio $\Omega$, podese escrever:

$$
\int \Phi(\mathrm{q}) \delta(\mathrm{s}, \mathrm{q}) \mathrm{d} \Omega_{\infty}=\Phi(\mathrm{s})
$$

Considerando as equações (2.76) e (2.77), pode-se dizer que a equação (2.75) é nula para todos os pontos do domínio fundamental, com exceção do ponto s. Assim, $\mathbf{w}^{\star}$ deve ser tal que satisfaça a equação:

$$
\nabla^{2} \nabla^{2} \mathrm{w}^{*}=0
$$

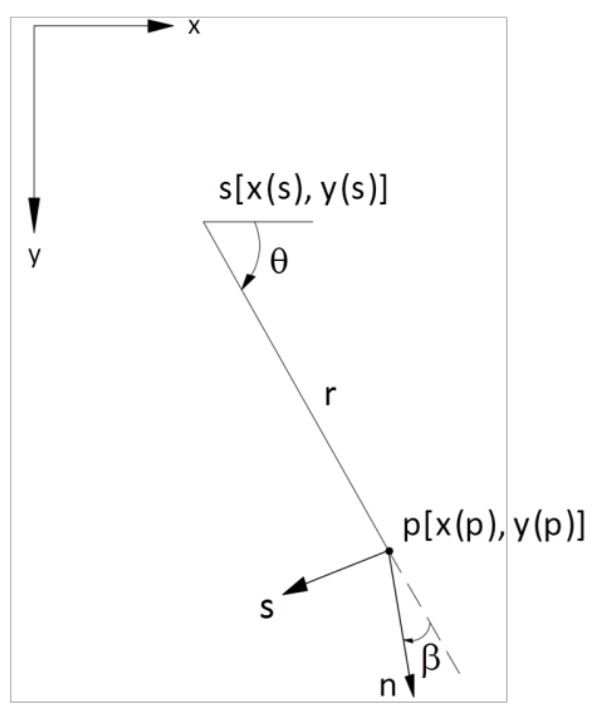

Figura 2.5 - Pontos de carregamento (s) e de deslocamento (p). 
Sendo um sistema de coordenadas polares de origem em s (ver Figura 2.5) e também a simetria existente, a equação (2.80) pode ser escrita da seguinte forma:

$$
\nabla^{2} \nabla^{2} \mathrm{w}^{*}=\left[\frac{\mathrm{d}^{2}}{\mathrm{dr} \mathrm{r}^{2}}+\frac{1}{\mathrm{r}} \frac{\mathrm{d}}{\mathrm{dr}}\right]\left[\frac{\mathrm{d}^{2} \mathrm{w}^{*}}{\mathrm{dr}^{2}}+\frac{1}{\mathrm{r}} \frac{\mathrm{dw}^{*}}{\mathrm{dr}}\right]=0
$$

ou seja:

$$
\left[\frac{\mathrm{d}^{4} \mathrm{w}^{*}}{\mathrm{dr}^{4}}+\frac{2}{\mathrm{r}} \frac{\mathrm{d}^{3} \mathrm{w}^{*}}{\mathrm{dr}^{3}}-\frac{1}{\mathrm{r}^{2}} \frac{\mathrm{d}^{2} \mathrm{w}^{*}}{\mathrm{dr}^{2}}+\frac{1}{\mathrm{r}^{3}} \frac{\mathrm{dw}^{*}}{\mathrm{dr}}\right]=0
$$

Fazendo integrações sucessivas, chega-se a:

$$
\mathrm{w}^{*}=A \cdot \ln r+B \cdot r^{2} \ln r+C \cdot r^{2}+E
$$

Levando em consideração a simetria do problema e o deslocamento finito, pode-se afirmar que a derivada $\mathrm{dw}^{*} / \mathrm{dr}$ é nula quando o raio é nulo, tem-se:

$$
A=0
$$

A constante $B$ se obtém da adoção de um círculo de raio $r$ com centro em s, sendo s o ponto de aplicação da carga unitária (Figura 2.6), onde surgirá, em seus limites, uma força cortante equivalente $V_{n}$ uniformemente distribuída para manter-se em equilíbrio. Assim:

$$
\mathrm{V}_{\mathrm{n}}=-\frac{1}{2 \pi \mathrm{r}}
$$

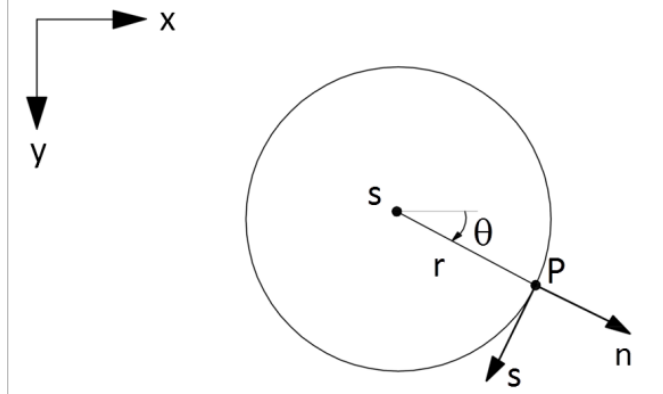

(a)

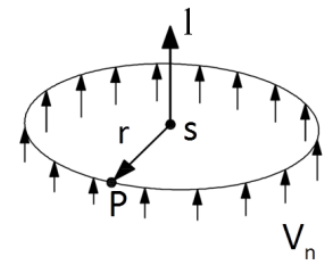

(b)

Figura 2.6 - Forcas verticais atuantes no círculo de raio r e centro no ponto s. 
Obtém-se a expressão de $V_{n}$ a partir da equação (2.74), considerando apenas em função de $r$ e $\beta=0$ :

$$
\mathrm{V}_{\mathrm{n}}=-\mathrm{D} \frac{\partial}{\partial \mathrm{r}}\left(\frac{\partial^{2} \mathrm{w}^{*}}{\partial \mathrm{r}^{2}}+\frac{1}{\mathrm{r}} \frac{\partial \mathrm{w}^{*}}{\partial \mathrm{r}}+\frac{1}{\mathrm{r}^{2}} \frac{\partial^{2} \mathrm{w}^{*}}{\partial \theta^{2}}\right)=-\frac{1}{2 \pi \mathrm{r}}
$$

Chega-se na constante B, substituindo-se a equação (2.83) na (2.86):

$$
\mathrm{B}=\frac{1}{8 \pi \mathrm{D}}
$$

Com estas constantes a equação (2.83) fica na seguinte forma:

$$
\mathrm{w}^{*}=\frac{1}{8 \pi \mathrm{D}} \mathrm{r}^{2} \ln r+C \mathrm{r}^{2}+\mathrm{E}
$$

Para as constantes $\mathrm{C}$ e E, aplica-se as condições de contorno da placa estudada. Como o caso estudado é a placa fundamental de raio infinito em questão, essas condições podem ser quaisquer. STERN (1979) e BEZINE (1978) adotam:

$$
C=\mathrm{E}=0
$$

Finalmente chegando em:

$$
\mathrm{w}^{*}=\frac{1}{8 \pi \mathrm{D}} \mathrm{r}^{2} \ln r
$$

Já DANSON (1979) adota:

$$
C=-\frac{1}{16 \pi D} \quad \text { e } \quad E=0
$$

Resultando na expressão utilizada neste trabalho:

$$
\mathrm{w}^{*}=\frac{1}{8 \pi \mathrm{D}} \mathrm{r}^{2}\left(\ln r-\frac{1}{2}\right)
$$

Conforme a Figura 2.7, determina-se também as derivadas das soluções fundamentais em relação à coordenada $\mathrm{m}$ de um sistema de referência cartesiano $(\mathrm{m}, \mathrm{u})$ com origem em $\mathrm{s}$. 


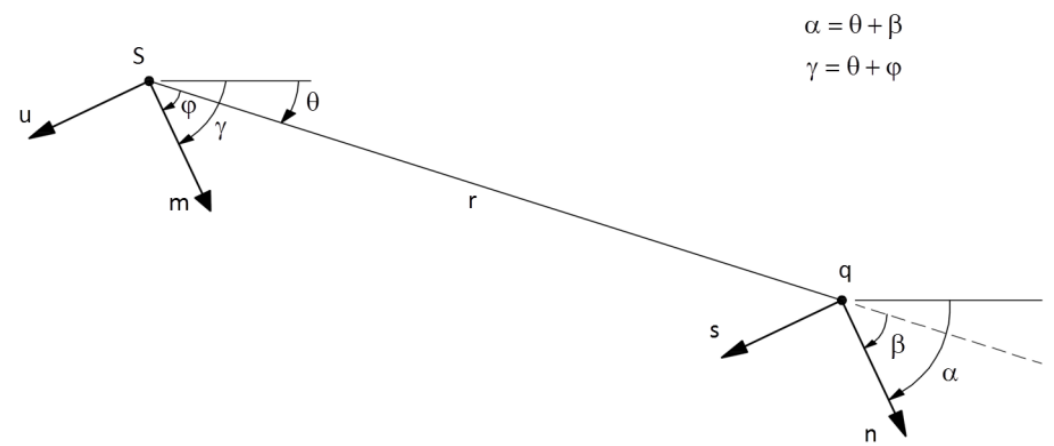

Figura 2.7 - Sistemas de coordenadas.

Tem-se, portanto a derivada do deslocamento no ponto s como:

$$
\frac{\partial \mathrm{w}^{*}}{\partial \mathrm{n}}=\frac{\partial \mathrm{w}^{*}}{\partial \mathrm{r}} \frac{\partial \mathrm{r}}{\partial \mathrm{n}}
$$

onde,

$$
\frac{\partial r}{\partial \mathrm{n}}=\frac{\partial \mathrm{r}}{\partial \mathrm{x}(\mathrm{q})} \frac{\partial \mathrm{x}(\mathrm{q})}{\partial \mathrm{n}}+\frac{\partial \mathrm{r}}{\partial \mathrm{y}(\mathrm{q})} \frac{\partial \mathrm{y}(\mathrm{q})}{\partial \mathrm{n}}
$$

Admitindo a equação (2.95):

$$
r(s, q)=\sqrt{(x(q)-x(s))^{2}+(y(q)-y(s))^{2}}
$$

as derivadas de $r$ em relação às coordenadas $x$ e y, seguem:

$$
\begin{gathered}
\frac{\partial r}{\partial x(q)}=r_{, x}=\frac{x(q)-x(s)}{r}=\cos \theta \\
\frac{\partial r}{\partial y(q)}=r_{, y}=\frac{y(q)-y(s)}{r}=\operatorname{sen} \theta
\end{gathered}
$$

As derivadas de segunda ordem são:

$$
\frac{\partial \mathrm{r}_{, \mathrm{x}}}{\partial \mathrm{x}(\mathrm{q})}=\frac{1-\mathrm{r}_{, \mathrm{x}}^{2}}{\mathrm{r}}
$$




$$
\begin{gathered}
\frac{\partial r_{, y}}{\partial x(q)}=-\frac{r_{, x} r_{, y}}{r} \\
\frac{\partial r_{, x}}{\partial y(q)}=-\frac{r_{, x} r_{, y}}{r} \\
\frac{\partial r_{, y}}{\partial y(q)}=\frac{1-r_{, y}^{2}}{r}
\end{gathered}
$$

Podendo ser escritas na forma indicial, como:

$$
\begin{gathered}
\frac{\partial r}{\partial x_{i}(q)}=r_{, i}=\frac{x_{i}(q)-x_{i}(s)}{r} \\
\frac{\partial^{2} r}{\partial x_{i}(q) \partial y_{i}(q)}=r_{, i j}=\frac{\delta_{j i}-r_{, i} r_{j}}{r}
\end{gathered}
$$

Onde, $\delta_{\mathrm{ji}}$ representa o delta de Kronecker, com valores:

$$
\begin{array}{ll}
\delta_{\mathrm{ji}}=0 & \mathrm{i} \neq \mathrm{j} \\
\delta_{\mathrm{ji}}=1 & \mathrm{i}=\mathrm{j}
\end{array}
$$

As derivadas em relação a $n$ valem:

$$
\begin{aligned}
& \frac{\partial \mathrm{x}(\mathrm{s})}{\partial \mathrm{n}}=\cos \alpha \\
& \frac{\partial \mathrm{y}(\mathrm{s})}{\partial \mathrm{n}}=\operatorname{sen} \alpha
\end{aligned}
$$

Quando combinadas as equações (2.96), (2.97), (2.98), (2.99), (2.100), (2.101), (2.106) e (2.107) com a equação (2.95), chega-se a:

$$
\frac{\partial \mathrm{r}}{\partial \mathrm{n}}=\cos \theta \cos \alpha+\operatorname{sen} \theta \operatorname{sen} \alpha=\cos \beta
$$

Portanto, 


$$
\frac{\partial \mathrm{w}^{*}}{\partial \mathrm{n}}=\frac{\mathrm{r}}{4 \pi \mathrm{D}} \ln r \cos \beta
$$

Considerando $\vec{r}$ o versor relacionado a $r$ :

$$
\frac{\partial \mathrm{w}^{*}}{\partial \mathrm{n}}=\frac{\mathrm{r}}{4 \pi \mathrm{D}} \ln r(\overrightarrow{\mathrm{n}} \cdot \overrightarrow{\mathrm{r}})=\frac{\mathrm{r}}{4 \pi \mathrm{D}} \ln r\left(\mathrm{n}_{, \mathrm{x}} \mathrm{r}_{\mathrm{x}}+\mathrm{n}_{\mathrm{y}, \mathrm{r}} \mathrm{r}_{\mathrm{y}}\right)=\frac{\mathrm{r}}{4 \pi \mathrm{D}} \ln r\left(\mathrm{n}_{\mathrm{i}} \mathrm{r}_{\mathrm{i}}\right)
$$

A partir de (2.92) obtêm-se as expressões dos esforços fundamentais, dadas por:

$$
\begin{gathered}
\mathrm{m}_{\mathrm{n}}^{*}=\frac{-1}{4 \pi}\left[(1+v) \ln r+(1-v) \cos ^{2} \beta+v\right] \\
\mathrm{q}_{\mathrm{n}}^{*}=-\frac{1}{2 \pi \mathrm{r}} \cos \beta \\
\mathrm{m}_{\mathrm{ns}}^{*}=\frac{1-v}{8 \pi} \operatorname{sen} 2 \beta \\
\mathrm{V}_{\mathrm{n}}^{*}=\frac{\cos \beta}{4 \pi \mathrm{r}}\left[2(1-v) \operatorname{sen}^{2} \beta-3+v\right]+\frac{1-v}{4 \pi \mathrm{R}} \cos 2 \beta
\end{gathered}
$$

onde,

$$
\begin{gathered}
\cos \beta=\vec{n} \cdot \vec{r}=n_{, x} r_{, x}+n_{, y} r_{, y}=n_{i} r_{i} \\
\operatorname{sen} \beta=-\vec{s} \cdot \vec{r}=-s_{, x} r_{, x}-s_{, y} r_{, y}=-s_{i} r_{i}
\end{gathered}
$$

portanto:

$$
\begin{gathered}
\frac{\partial \mathrm{w}^{*}}{\partial \mathrm{n}}=\frac{\mathrm{r}}{4 \pi \mathrm{D}} \ln r(\overrightarrow{\mathrm{n}} \cdot \overrightarrow{\mathrm{r}}) \\
\mathrm{q}_{\mathrm{n}}^{*}=-\frac{1}{2 \pi \mathrm{r}}(\overrightarrow{\mathrm{n}} \cdot \overrightarrow{\mathrm{r}}) \\
\mathrm{m}_{\mathrm{n}}^{*}=\frac{-1}{4 \pi}\left[(1+v) \ln r+(1-v)(\overrightarrow{\mathrm{n}} \cdot \overrightarrow{\mathrm{r}})^{2}+v\right] \\
\mathrm{m}_{\mathrm{ns}}^{*}=-\frac{1-v}{4 \pi}(\overrightarrow{\mathrm{n}} \cdot \overrightarrow{\mathrm{r}})(\overrightarrow{\mathrm{s}} \cdot \overrightarrow{\mathrm{r}})
\end{gathered}
$$




$$
V_{n}^{*}=\frac{(\vec{n} \cdot \vec{r})}{4 \pi r}\left[2(1-v)(\vec{s} \cdot \vec{r})^{2}-3+v\right]+\frac{1-v}{4 \pi R} 2(\vec{n} \cdot \vec{r})(\vec{s} \cdot \vec{r})
$$

A derivada do deslocamento transversal em relação à direção $m$ no ponto $s$ é dada por:

$$
\frac{\partial \mathrm{w}^{*}}{\partial \mathrm{m}}=\frac{\partial \mathrm{w}^{*}}{\partial \mathrm{r}} \frac{\partial \mathrm{r}}{\partial \mathrm{m}}
$$

onde,

$$
\frac{\partial \mathrm{r}}{\partial \mathrm{m}}=\frac{\partial \mathrm{r}}{\partial \mathrm{x}(\mathrm{s})} \frac{\partial \mathrm{x}(\mathrm{s})}{\partial \mathrm{m}}+\frac{\partial \mathrm{r}}{\partial \mathrm{y}(\mathrm{s})} \frac{\partial \mathrm{y}(\mathrm{s})}{\partial \mathrm{m}}
$$

A partir de (2.94), as derivadas de $r$ em relação às coordenadas $x$ e y podem ser escritas como:

$$
\begin{gathered}
\frac{\partial r}{\partial x(s)}=-r_{, x}=-\frac{x(q)-x(s)}{r}=-\cos \theta \\
\frac{\partial r}{\partial y(s)}=-r_{, y}=-\frac{y(q)-y(s)}{r}=-\operatorname{sen} \theta
\end{gathered}
$$

As derivadas de segunda ordem são:

$$
\begin{gathered}
\frac{\partial r_{, x}}{\partial x(s)}=-\frac{1-r_{, x}^{2}}{r} \\
\frac{\partial r_{, y}}{\partial x(s)}=\frac{r_{, x} r_{, y}}{r} \\
\frac{\partial r_{, x}}{\partial y(s)}=\frac{r_{, x} r_{, y}}{r} \\
\frac{\partial r_{, y}}{\partial y(s)}=-\frac{1-r_{, y}^{2}}{r}
\end{gathered}
$$

Podendo ser escritas na forma indicial, como: 


$$
\begin{gathered}
\frac{\partial r}{\partial x_{i}(s)}=-r_{, i}=-\frac{x_{i}(q)-x_{i}(s)}{r} \\
\frac{\partial^{2} r}{\partial x_{i}(s) \partial y_{i}(s)}=-r_{, i j}=-\frac{\delta_{j i}-r_{, i} r_{j}}{r}
\end{gathered}
$$

Podem ser escritas também as seguintes relações:

$$
\begin{aligned}
& \frac{\partial \mathrm{x}(\mathrm{s})}{\partial \mathrm{m}}=\cos \gamma=\mathrm{m}_{\mathrm{x}} \\
& \frac{\partial \mathrm{y}(\mathrm{s})}{\partial \mathrm{m}}=\operatorname{sen} \gamma=\mathrm{m}_{\mathrm{y}}
\end{aligned}
$$

Substituindo (2.132) e (2.133) em (2.123) chega-se a:

$$
\frac{\partial \mathrm{r}}{\partial \mathrm{m}}=\cos \gamma(-\cos \theta)+\operatorname{sen} \gamma(-\operatorname{sen} \theta)=-\cos \varphi
$$

Portanto,

$$
\begin{gathered}
\frac{\partial \mathrm{w}^{*}}{\partial \mathrm{m}}=\frac{\mathrm{r}}{4 \pi \mathrm{D}} \ln r(-\cos \varphi)=\frac{\mathrm{r}}{4 \pi \mathrm{D}} \ln r(\overrightarrow{\mathrm{m}} \cdot \overrightarrow{\mathrm{r}})=\frac{\mathrm{r}}{4 \pi \mathrm{D}} \ln r\left(\mathrm{~m}_{, \mathrm{x}} \mathrm{r}_{, \mathrm{x}}+\mathrm{m}_{, \mathrm{y}, \mathrm{y}}\right) \\
=\frac{\mathrm{r}}{4 \pi \mathrm{D}} \ln r\left(\mathrm{~m}_{\mathrm{i}} \mathrm{r}_{\mathrm{i}}\right)
\end{gathered}
$$

Escrevendo as demais expressões na forma indicial, resulta-se em:

$$
\begin{gathered}
\frac{\partial}{\partial \mathrm{m}}\left(\frac{\partial \mathrm{w}^{*}}{\partial \mathrm{n}}\right)=\frac{-1}{4 \pi \mathrm{D}}\left[\left(\mathrm{m}_{\mathrm{i}} \mathrm{r}_{\mathrm{i}}\right)\left(\mathrm{n}_{\mathrm{i}} \mathrm{r}_{\mathrm{i}}\right)+\left(\mathrm{m}_{\mathrm{i}} \mathrm{n}_{\mathrm{i}}\right) \ln r\right] \\
\frac{\partial \mathrm{q}_{\mathrm{n}}^{*}}{\partial \mathrm{m}}=\frac{1}{2 \pi \mathrm{r}^{2}}\left[\left(\mathrm{~m}_{\mathrm{i}} \mathrm{n}_{\mathrm{i}}\right)-2\left(\mathrm{~m}_{\mathrm{i}} \mathrm{r}_{\mathrm{i}}\right)\left(\mathrm{n}_{\mathrm{i}} \mathrm{r}_{\mathrm{i}}\right)\right] \\
\frac{\partial \mathrm{m}_{\mathrm{n}}^{*}}{\partial \mathrm{m}}=\frac{-1}{4 \pi \mathrm{r}}\left\{(1+v)\left(\mathrm{m}_{\mathrm{i}} \mathrm{r}_{\mathrm{i}}\right)+2(1-v)\left(\mathrm{n}_{\mathrm{i}} \mathrm{r}_{\mathrm{i}}\right)\left[\left(\mathrm{m}_{\mathrm{i}} \mathrm{n}_{\mathrm{i}}\right)-\left(\mathrm{m}_{\mathrm{i}} \mathrm{r}_{\mathrm{i}}\right)\left(\mathrm{n}_{\mathrm{i}} \mathrm{r}_{\mathrm{i}}\right)\right]\right\} \\
\frac{\partial \mathrm{m}_{\mathrm{ns}}^{*}}{\partial \mathrm{m}}=\frac{-(1-v)}{4 \pi \mathrm{r}}\left[\left(\mathrm{m}_{\mathrm{i}} \mathrm{n}_{\mathrm{i}}\right)\left(\mathrm{s}_{\mathrm{i}} \mathrm{r}_{\mathrm{i}}\right)+\left(\mathrm{m}_{\mathrm{i}} \mathrm{s}_{\mathrm{i}}\right)\left(\mathrm{n}_{\mathrm{i}} \mathrm{r}_{\mathrm{i}}\right)-2\left(\mathrm{~m}_{\mathrm{i}} \mathrm{r}_{\mathrm{i}}\right)\left(\mathrm{n}_{\mathrm{i}} \mathrm{r}_{\mathrm{i}}\right)\left(\mathrm{s}_{\mathrm{i}} \mathrm{r}_{\mathrm{i}}\right)\right]
\end{gathered}
$$




$$
\begin{aligned}
\frac{\partial V_{n}^{*}}{\partial m}=\frac{-1}{4 \pi r^{2}}\{2 & (1-v)\left(s_{i} r_{i}\right)\left[4\left(s_{i} r_{i}\right)\left(m_{i} r_{i}\right)\left(n_{i} r_{i}\right)-2\left(m_{i} s_{i}\right)\left(n_{i} r_{i}\right)-\left(s_{i} r_{i}\right)\left(m_{i} n_{i}\right)\right] \\
& +(3-v)\left[\left(m_{i} n_{i}\right)-2\left(m_{i} r_{i}\right)\left(n_{i} r_{i}\right)\right] \\
& \left.+\frac{(1-v)}{\pi R r}\left(s_{i} r_{i}\right)\left[\left(m_{i} s_{i}\right)-\left(m_{i} r_{i}\right)\left(r_{i} s_{i}\right)\right]\right\}
\end{aligned}
$$

Estas expressões também podem ser escritas como:

$$
\begin{gathered}
\frac{\partial \mathrm{w}^{*}}{\partial \mathrm{m}}=\frac{\mathrm{r}}{4 \pi \mathrm{D}} \ln r(\overrightarrow{\mathrm{m}} \cdot \overrightarrow{\mathrm{r}}) \\
\frac{\partial}{\partial \mathrm{m}}\left(\frac{\partial \mathrm{w}^{*}}{\partial \mathrm{n}}\right)=\frac{-1}{4 \pi \mathrm{D}}[(\overrightarrow{\mathrm{m}} \cdot \overrightarrow{\mathrm{r}})(\overrightarrow{\mathrm{n}} \cdot \overrightarrow{\mathrm{r}})+(\overrightarrow{\mathrm{m}} \cdot \overrightarrow{\mathrm{n}}) \ln r] \\
\frac{\partial \mathrm{q}_{\mathrm{n}}^{*}}{\partial \mathrm{m}}=\frac{1}{2 \pi \mathrm{r}^{2}}[(\overrightarrow{\mathrm{m}} \cdot \overrightarrow{\mathrm{n}})-2(\overrightarrow{\mathrm{m}} \cdot \overrightarrow{\mathrm{r}})(\overrightarrow{\mathrm{n}} \cdot \overrightarrow{\mathrm{r}})] \\
\frac{\partial \mathrm{m}_{\mathrm{n}}^{*}}{\partial \mathrm{m}}=\frac{-1}{4 \pi \mathrm{r}}\{(1+v)(\overrightarrow{\mathrm{m}} \cdot \overrightarrow{\mathrm{r}})+2(1-v)(\overrightarrow{\mathrm{n}} \cdot \overrightarrow{\mathrm{r}})[(\overrightarrow{\mathrm{m}} \cdot \overrightarrow{\mathrm{n}})-(\overrightarrow{\mathrm{m}} \cdot \overrightarrow{\mathrm{r}})(\overrightarrow{\mathrm{n}} \cdot \overrightarrow{\mathrm{r}})]\} \\
\frac{\partial \mathrm{m}_{\mathrm{n}}^{*}}{\partial \mathrm{m}}=\frac{-(1-v)}{4 \pi \mathrm{r}}[(\overrightarrow{\mathrm{m}} \cdot \overrightarrow{\mathrm{n}})(\overrightarrow{\mathrm{s}} \cdot \overrightarrow{\mathrm{r}})+(\overrightarrow{\mathrm{m}} \cdot \overrightarrow{\mathrm{s}})(\overrightarrow{\mathrm{n}} \cdot \overrightarrow{\mathrm{r}})-2(\overrightarrow{\mathrm{m}} \cdot \overrightarrow{\mathrm{r}})(\overrightarrow{\mathrm{n}} \cdot \overrightarrow{\mathrm{r}})(\overrightarrow{\mathrm{s}} \cdot \overrightarrow{\mathrm{r}})]
\end{gathered}
$$




\section{EQUAÇÕES INTEGRAIS PARA FLEXÃO DE PLACAS}

\subsection{Introdução}

Neste capítulo, utilizando as equações e expressões obtidas no capítulo anterior e utilizando o teorema de Betti para uma placa submetida a dois carregamentos distintos, $\mathrm{g} \mathrm{e} \mathrm{g}^{*}$, que resultam em dois estados de tensão e de deslocamento correspondentes e efetuando integrações por partes sobre a expressão resultante da aplicação do teorema de Betti, obtêm-se as equações integrais em um ponto qualquer do domínio da placa. Estas equações integrais referentes ao deslocamento transversal $w(s)$ e às suas derivadas direcionais $\partial \mathrm{w}(\mathrm{s}) / \partial \mathrm{n}$ e $\partial \mathrm{w}(\mathrm{s}) / \partial \mathrm{s}$ de um ponto do contorno são relacionados aos esforços no contorno e ações de domínio, conforme PAIVA(1987) e OLIVEIRA NETO(1998).

\subsection{Equação integral para um ponto do domínio}

Seja uma placa isótropa qualquer, de contorno $\Gamma$ e domínio $\Omega$, contida em uma placa infinita de contorno $\Gamma_{\infty}$ e domínio $\Omega_{\infty}$. Ao aplicar a placa infinita dois carregamentos distintos, g distribuído em uma região de área $\Omega_{\mathrm{g}}, \mathrm{e} \mathrm{g}^{*}$ carregamento correspondente à solução fundamental, obtêm-se dois estados de tensão, $\sigma_{i j}$ e $\sigma_{i j}^{*}$, e dois de deformação, $\varepsilon_{i j}$ e $\varepsilon_{i j}^{*}$, correspondentes.

O teorema da reciprocidade de Betti fornece a seguinte igualdade:

$$
\int_{v} \sigma_{i j}^{*} \varepsilon_{i j} d v=\int_{v} \sigma_{i j} \varepsilon_{i j}^{*} d v \quad i, j=1,2,3 \ldots
$$

O termo da direita da equação (2.1), denominado aqui de $U$, pode ser escrito via notação clássica, da seguinte forma:

$$
U=\int_{v}\left[\sigma_{x} \varepsilon_{x}^{*}+\sigma_{y} \varepsilon_{y}^{*}+\sigma_{z} \varepsilon_{z}^{*}+2\left(\tau_{x y} \varepsilon_{x y}^{*}+\tau_{x z} \varepsilon_{x z}^{*}+\tau \sigma_{y z} \varepsilon_{y z}^{*}\right)\right] d V
$$


Desprezando as tensões relativas à direção z, normal ao plano da placa da equação (3.2), pode-se escrever:

$$
U=\int_{v}\left(\sigma_{x} \varepsilon_{x}^{*}+\sigma_{y} \varepsilon_{y}^{*}+2 \tau_{x y} \varepsilon_{x y}^{*}\right) d V
$$

Substituindo agora os valores de tensões e de deformações dados pelas equações do capítulo anterior (equações (2.11), (2.12), (2.13), (2.18), (2.19), (2.20), obtém-se a integral do primeiro termo de U:

$$
\int_{v} \sigma_{x} \varepsilon_{x}^{*} d V=\int_{v}-\frac{E}{\left(1-v^{2}\right)}\left\{-z^{2}\left(\frac{\partial^{2} w}{\partial x^{2}}+v \frac{\partial^{2} w}{\partial y^{2}}\right) \frac{\partial^{2} w}{\partial x^{2}}\right\} d V
$$

Após a integração desta equação na espessura, a integral torna-se em integral sobre o domínio $\Omega$ :

$$
\int_{v} \sigma_{x} \varepsilon_{x}^{*} d V=\int_{\Omega} D\left(\frac{\partial^{2} w}{\partial x^{2}}+v \frac{\partial^{2} w}{\partial y^{2}}\right) \frac{\partial^{2} w^{*}}{\partial x^{2}} d \Omega
$$

De forma análoga trabalha-se com os dois termos restantes da equação (3.3), obtendo-se:

$$
\int_{\Omega} D\left[\left(\frac{\partial^{2} w}{\partial x^{2}}+v \frac{\partial^{2} w}{\partial y^{2}}\right) \frac{\partial^{2} w^{*}}{\partial x^{2}}+D\left(\frac{\partial^{2} w}{\partial y^{2}}+v \frac{\partial^{2} w}{\partial x^{2}}\right) \frac{\partial^{2} w^{*}}{\partial y^{2}}+2 D(1-v) \frac{\partial^{2} w}{\partial x \partial y} \frac{\partial^{2} w^{*}}{\partial x \partial y}\right] d \Omega
$$

Reescrevendo as equações (2.21), (2.22) e (2.23), obtém-se integral de volume representada na equação (3.3) transformada em integral sobre o domínio $\Omega$ :

$$
U=\int_{\Omega}\left[-m_{x} \frac{\partial^{2} w^{*}}{\partial x^{2}}-m_{y} \frac{\partial^{2} w^{*}}{\partial y^{2}}-2 m_{x y} \frac{\partial^{2} w^{*}}{\partial x \partial y}\right] d \Omega
$$

A fim de transformar em integral sobre o contorno, trabalha-se com cada parcela individualmente. Integrando por partes a primeira parcela da equação (3.7), na direção x e obtém-se: 


$$
-\int_{\Omega} m_{x} \frac{\partial^{2} w^{*}}{\partial x^{2}} d \Omega=-\int_{\Gamma} m_{x} \frac{\partial w^{*}}{\partial x} \eta_{x} d \Gamma+\int_{\Omega} \frac{\partial m_{x}}{\partial x} \frac{\partial w^{*}}{\partial x} d \Omega
$$

Sendo $\eta_{x}$ o co-seno diretor do versor normal ao contorno na direção $\mathrm{x}$ (Figura 3.1) .

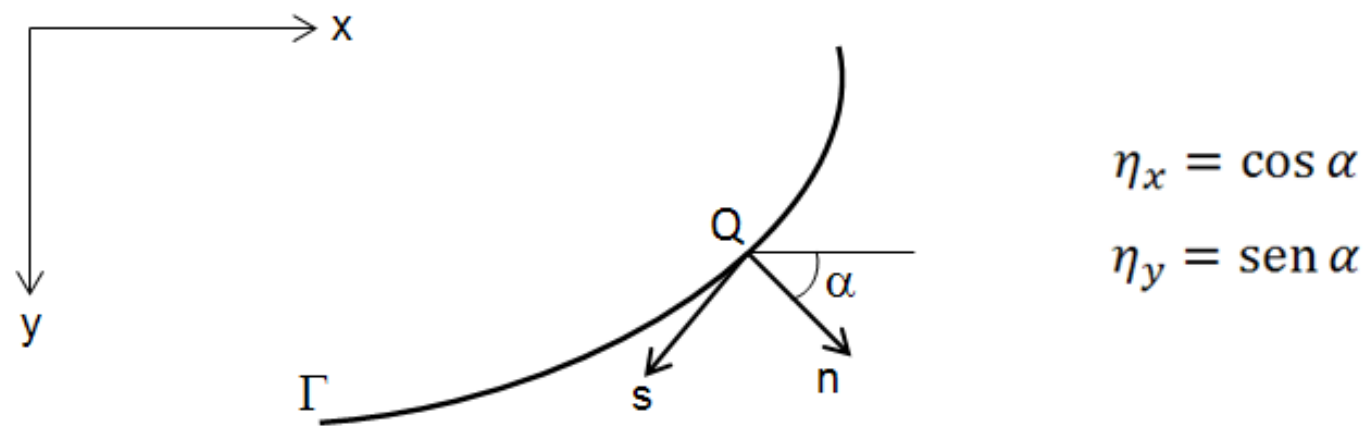

Figura 3.1 - Coordenadas (n, s) no ponto $Q$ do contorno

Integrando por partes a integral de domínio da segunda parcela da equação (3.8), tem-se:

$$
-\int_{\Omega} m_{x} \frac{\partial^{2} w^{*}}{\partial x^{2}} d \Omega=\int_{\Gamma}\left(-m_{x} \frac{\partial w^{*}}{\partial x} \cos \alpha+\frac{\partial m_{x}}{\partial x} w^{*} \cos \alpha\right) d \Gamma-\int_{\Omega} \frac{\partial^{2} m_{x}}{\partial x^{2}} w^{*} d \Omega
$$

Integrando por partes agora a integral de domínio da segunda parcela da equação (3.7), de forma análoga, na direção y, consegue-se:

$$
-\int_{\Omega} m_{y} \frac{\partial^{2} w^{*}}{\partial y^{2}} d \Omega=-\int_{\Gamma} m_{y} \frac{\partial w^{*}}{\partial y} \eta_{y} d \Gamma+\int_{\Omega} \frac{\partial m_{y}}{\partial y} \frac{\partial w^{*}}{\partial y} d \Omega
$$

E finalmente, integrando por partes a segunda parcela da equação (3.10), obtém-se:

$$
-\int_{\Omega} m_{y} \frac{\partial^{2} w^{*}}{\partial y^{2}} d \Omega=\int_{\Gamma}\left(-m_{y} \frac{\partial w^{*}}{\partial y} \operatorname{sen} \alpha+\frac{\partial m_{y}}{\partial y} w^{*} \operatorname{sen} \alpha\right) d \Gamma-\int_{\Omega} \frac{\partial^{2} m_{y}}{\partial y^{2}} w^{*} d \Omega
$$

Reescrevendo agora a terceira parte da equação (3.7), chega-se a: 


$$
-2 \int_{\Omega} m_{x y} \frac{\partial^{2} w^{*}}{\partial x \partial y} d \Omega=-\int_{\Omega} m_{x y} \frac{\partial^{2} w^{*}}{\partial x \partial y} d \Omega-\int_{\Omega} m_{x y} \frac{\partial^{2} w^{*}}{\partial x \partial y} d \Omega
$$

E agora integrando por partes as duas parcelas, em relação às direções $\mathrm{X}$ e y, tem-se:

$$
\begin{aligned}
-2 \int_{\Omega} m_{x y} \frac{\partial^{2} w^{*}}{\partial x \partial y} d \Omega & \\
= & -\int_{\Gamma} m_{x y} \frac{\partial w^{*}}{\partial y} \cos \alpha d \Gamma+\int_{\Omega} \frac{\partial m_{x y}}{\partial x} \frac{\partial w^{*}}{\partial y} d \Omega \\
& -\int_{\Gamma} m_{x y} \frac{\partial w^{*}}{\partial x} \operatorname{sen} \alpha d \Gamma+\int_{\Omega} \frac{\partial m_{x y}}{\partial y} \frac{\partial w^{*}}{\partial x} d \Omega
\end{aligned}
$$

Integrando por partes as integrais de domínio da equação (3.13), obtémse:

$$
\begin{aligned}
-2 \int_{\Omega} m_{x y} \frac{\partial^{2} w^{*}}{\partial x \partial y} d \Omega & \\
= & \int_{\Gamma}\left(-m_{x y} \frac{\partial w^{*}}{\partial y} \cos \alpha-m_{x y} \frac{\partial w^{*}}{\partial x} \operatorname{sen} \alpha+\frac{\partial m_{x y}}{\partial x} w^{*} \operatorname{sen} \alpha\right. \\
& \left.+\frac{\partial m_{x y}}{\partial y} w^{*} \cos \alpha\right) d \Gamma-\int_{\Omega} 2 \frac{\partial^{2} m_{x y}}{\partial x \partial y} w^{*} d \Omega
\end{aligned}
$$

Com as equações (3.9), (3.11) e (3.14) reescreve-se U como:

$$
\begin{gathered}
U=-\int_{\Gamma}\left(m_{x} \frac{\partial w^{*}}{\partial x} \cos \alpha+m_{y} \frac{\partial w^{*}}{\partial y} \operatorname{sen} \alpha+m_{x y} \frac{\partial w^{*}}{\partial y} \cos \alpha+m_{x y} \frac{\partial w^{*}}{\partial x} \operatorname{sen} \alpha\right) d \Gamma \\
+\int_{\Gamma}\left[\left(\frac{\partial m_{x}}{\partial \mathrm{x}}+\frac{\partial m_{x y}}{\partial \mathrm{y}}\right) \cos \alpha+\left(\frac{\partial m_{y}}{\partial \mathrm{y}}+\frac{\partial m_{x y}}{\partial \mathrm{x}}\right) \operatorname{sen} \alpha\right] w^{*} d \Gamma \\
-\int_{\Omega}\left(\frac{\partial^{2} m_{x}}{\partial \mathrm{x}^{2}}+\frac{\partial^{2} m_{x}}{\partial \mathrm{y}^{2}}+2 \frac{\partial^{2} m_{x y}}{\partial \mathrm{x} \partial \mathrm{y}}\right) w^{*} d \Omega
\end{gathered}
$$

E usando as relações das equações (2.1) até a (2.8), escreve-se:

$$
\begin{gathered}
U=-\int_{\Gamma}\left(m_{x} \frac{\partial w^{*}}{\partial x} \cos \alpha+m_{y} \frac{\partial w^{*}}{\partial y} \operatorname{sen} \alpha+m_{x y} \frac{\partial w^{*}}{\partial y} \cos \alpha+m_{x y} \frac{\partial w^{*}}{\partial x} \operatorname{sen} \alpha\right) d \Gamma \\
+\int_{\Gamma} \mathrm{q}_{\mathrm{n}} \mathrm{w}^{*} \mathrm{~d} \Gamma+\int_{\Omega_{\mathrm{g}}} \mathrm{gw}^{*} \mathrm{~d} \Omega_{\mathrm{g}}
\end{gathered}
$$


Sendo $\Omega_{\mathrm{g}}$, a região carregada da placa.

Tomando as relações abaixo:

$$
\begin{aligned}
& \frac{\partial \mathrm{w}^{*}}{\partial x}=\frac{\partial \mathrm{w}^{*}}{\partial n} \cos \alpha-\frac{\partial \mathrm{w}^{*}}{\partial s} \operatorname{sen} \alpha \\
& \frac{\partial \mathrm{w}^{*}}{\partial y}=\frac{\partial \mathrm{w}^{*}}{\partial n} \operatorname{sen} \alpha+\frac{\partial \mathrm{w}^{*}}{\partial s} \cos \alpha
\end{aligned}
$$

Substituindo as equações (3.17) e (3.18) na equação (3.16), tem-se:

$$
\begin{aligned}
U=-\int_{\Gamma}\left\{\left(m_{x} \cos ^{2} \alpha+m_{y} \operatorname{sen}^{2} \alpha+2 m_{x y} \cos \alpha \operatorname{sen} \alpha\right) \frac{\partial \mathrm{w}^{*}}{\partial n}\right. \\
\left.+\left[\left(m_{y}-m_{x}\right) \operatorname{sen} \alpha \cos \alpha+m_{x y}\left(\cos ^{2} \alpha-\operatorname{sen}^{2} \alpha\right)\right] \frac{\partial \mathrm{w}^{*}}{\partial s}\right\} d \Gamma \\
+\int_{\Gamma} \mathrm{q}_{\mathrm{n}} \mathrm{w}^{*} \mathrm{~d} \Gamma+\int_{\Omega_{\mathrm{g}}} \mathrm{g} \mathrm{w}^{*} \mathrm{~d} \Omega_{\mathrm{g}}
\end{aligned}
$$

Assim, a partir das equações (2.32), (2.33), (2.34) e (2.35), U pode ser simplificado como:

$$
U=-\int_{\Gamma}\left(m_{n} \frac{\partial w^{*}}{\partial n}+m_{n s} \frac{\partial w^{*}}{\partial s}-q_{n} \mathrm{w}^{*}\right) d \Gamma+\int_{\Omega_{\mathrm{g}}} \mathrm{gw}^{*} \mathrm{~d} \Omega_{\mathrm{g}}
$$

Integrando por partes o termo de $m_{n s}$, obtém-se:

$$
\int_{\Gamma} m_{n s} \frac{\partial w^{*}}{\partial s} d \Gamma=\sum m_{n s} \mathrm{w}^{*} \mid \begin{aligned}
& \Gamma_{2} \\
& \Gamma_{1}
\end{aligned}-\int_{\Gamma} \frac{\partial \mathrm{m}_{\mathrm{ns}}}{\partial \mathrm{s}} \mathrm{w}^{*} \mathrm{~d} \Gamma
$$

Sendo $\Gamma_{1}$ e $\Gamma_{2}$ os limites do contorno onde se faz a integração. Para um contorno fechado e sem apresentar cantos, anula-se a primeira parcela. E na existência de cantos, reações de canto surgirão, denominadas aqui de $\mathrm{R}$. 




Figura 3.2 - Cantos da placa e momentos volventes resultantes

Na Figura 3.2 pode-se observar uma placa e os diversos cantos com os respectivos momentos volventes resultantes.

O primeiro termo da equação (3.21) será admitido como um somatório para todos os lados da placa e, ao tomar dois lados desta placa para demonstração, tem-se:

$$
\left(m_{n s_{i}}^{-} w_{i}^{*}-m_{n s_{i-1}}^{+} w_{i-1}^{*}\right)+\left(m_{n s_{i+1}}^{-} w_{i+1}^{*}-m_{n s_{i}}^{+} w_{i}^{*}\right)
$$

Para o canto i temos:

$$
-\left(m_{n s_{i}}^{-}-m_{n s_{i}}^{-}\right) w_{i}^{*}=-R_{c_{i}} w_{i}^{*}
$$

Escrevendo o primeiro termo da equação (3.21) a partir da equação (3.23), tem-se:

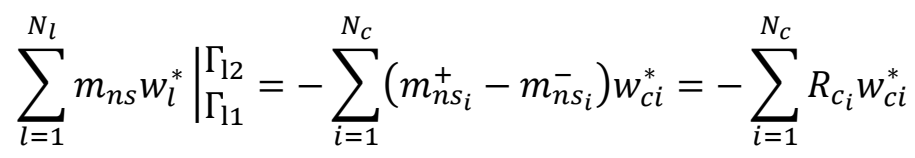

Em que,

$N_{l}$ : número de lados da placa;

$N_{c}$ : número de cantos da placa;

$w_{c i}^{*}$ : deslocamento fundamental no canto i;

$\Gamma_{11}$ e $\Gamma_{12}$ : limites de cada lado da placa. 
Escreve-se então a equação (3.21) como:

$$
\int_{\Gamma} m_{n s} \frac{\partial w^{*}}{\partial s} d \Gamma=-\sum_{i=1}^{N_{c}} R_{c_{i}} w_{c i}^{*}-\int_{\Gamma} \frac{\partial m_{n s}}{\partial s} \mathrm{w}^{*} d \Gamma
$$

Que quando substituída na equação (3.20), resulta:

$U=\int_{\Gamma}\left(\mathrm{q}_{\mathrm{n}} \mathrm{w}^{*}+\frac{\partial m_{n s}}{\partial s} \mathrm{w}^{*}-\mathrm{m}_{\mathrm{n}} \frac{\partial w^{*}}{\partial n}\right) d \Gamma+\sum_{i=1}^{N_{c}} R_{c_{i}} w_{c i}^{*}+\int_{\Omega_{\mathrm{g}}} \mathrm{gw}^{*} \mathrm{~d} \Omega_{\mathrm{g}}$

De posse da equação (2.38), obtém-se:

$$
U=\int_{\Gamma}\left(\mathrm{V}_{\mathrm{n}} \mathrm{w}^{*}-\mathrm{m}_{\mathrm{n}} \frac{\partial w^{*}}{\partial n}\right) d \Gamma+\sum_{i=1}^{N_{c}} R_{c_{i}} w_{c i}^{*}+\int_{\Omega_{\mathrm{g}}} \mathrm{gw}^{*} \mathrm{~d} \Omega_{\mathrm{g}}
$$

Novamente, considerando $\Omega_{\mathrm{g}}$, a região carregada da placa.

O termo da esquerda da equação (2.1) é obtido pelo processo análogo:

$$
U^{\prime}=-\int_{\Gamma}\left(\mathrm{m}_{\mathrm{n}}^{*} \frac{\partial w}{\partial n}+\mathrm{m}_{\mathrm{ns}}^{*} \frac{\partial w}{\partial s}-\mathrm{q}_{\mathrm{n}}^{*} \mathrm{w}\right) d \Gamma+\int_{\Omega} \mathrm{g}^{*} \mathrm{wd} \Omega
$$

Reescrevendo a equação (2.1) em função das equações (3.27) e (3.28) encontradas, tem-se a expressão de Betti aplicado a placas:

$$
\begin{aligned}
-\int_{\Gamma}\left[\mathrm{m}_{\mathrm{ns}}^{*} \frac{\partial w}{\partial s}\right. & \left.+\mathrm{m}_{\mathrm{n}}^{*} \frac{\partial w}{\partial n}-\mathrm{q}_{\mathrm{n}}^{*} \mathrm{w}\right] d \Gamma+\int_{\Omega} \mathrm{g}^{*} \mathrm{wd} \Omega \\
& =\int_{\Gamma}\left[\mathrm{V}_{\mathrm{n}} \mathrm{w}^{*}-\mathrm{m}_{\mathrm{n}} \frac{\partial w^{*}}{\partial n}\right] d \Gamma+\sum_{i=1}^{N_{c}} R_{c_{i}} w_{c i}^{*}+\int_{\Omega_{\mathrm{g}}} \mathrm{gw}^{*} \mathrm{~d} \Omega_{\mathrm{g}}
\end{aligned}
$$


Ao reescrever a equação (3.29) de forma a relacionar cada variável como função dos pontos na placa, tem-se:

$$
\begin{aligned}
\int_{\Gamma}\left[\mathrm{q}_{\mathrm{n}}^{*}(\mathrm{~s}, \mathrm{Q}) \mathrm{w}(\mathrm{Q})\right. & \left.-\mathrm{m}_{\mathrm{n}}^{*}(\mathrm{~s}, \mathrm{Q}) \frac{\partial w}{\partial n}(Q)-\mathrm{m}_{\mathrm{ns}}^{*}(\mathrm{~s}, \mathrm{Q}) \frac{\partial w}{\partial s}(Q)\right] d \Gamma(\mathrm{Q}) \\
& +\int_{\Omega} \mathrm{g}^{*}(\mathrm{~s}, \mathrm{q}) \mathrm{w}(\mathrm{q}) \mathrm{d} \Omega(\mathrm{q}) \\
& =\int_{\Gamma}\left[\mathrm{V}_{\mathrm{n}}(\mathrm{Q}) \mathrm{w}^{*}(\mathrm{~s}, \mathrm{Q})-\mathrm{m}_{\mathrm{n}}(\mathrm{Q}) \frac{\partial w^{*}}{\partial n}(\mathrm{~s}, \mathrm{Q})\right] d \Gamma(\mathrm{Q})+\sum_{i=1}^{N_{c}} R_{c_{i}}(Q) w_{c i}^{*}(\mathrm{~s}, \mathrm{Q}) \\
& +\int_{\Omega_{\mathrm{g}}} \mathrm{g}(\mathrm{q}) \mathrm{w}^{*}(\mathrm{~s}, \mathrm{q}) \mathrm{d} \Omega_{\mathrm{g}}(\mathrm{q})
\end{aligned}
$$

Onde as variáveis relacionam-se respectivamente por:

a) os esforços e os deslocamentos correspondentes ao carregamento $\mathrm{g}$ dependem apenas do ponto de campo Q;

b) a carga unitária fundamental $\mathrm{g}^{*}$ aplicada no ponto $\mathrm{s}$ do domínio da placa é representada pela função delta de $\operatorname{Dirac} \delta(s, q)$ e, portanto, é função dos pontos de colocação de carga s e de campo Q;

$$
\int_{\mathrm{g}} \delta(s, q) w(q) \mathrm{d} \Omega_{\mathrm{g}}(\mathrm{q})=\mathrm{w}(\mathrm{s})
$$

c) os esforços e os deslocamentos correspondentes à carga $g^{\star}$ dependem dos pontos de carga s e de deslocamento $Q$.

Com isto a expressão do teorema de Betti aplicado a placas fica representada na equação (3.32), onde tem-se a equação integral do deslocamento transversal w de um ponto $s$ do domínio da placa, expressa em função dos esforços $\mathrm{V}_{\mathrm{n}}(\mathrm{Q})$ e $\mathrm{m}_{\mathrm{n}}(\mathrm{Q})$ e dos deslocamentos $\mathrm{w}(\mathrm{Q}), \frac{\partial w}{\partial n}(Q)$ e $\frac{\partial w}{\partial s}(Q)$ de pontos do contorno, utilizando-se as soluções fundamentais obtidas no capítulo anterior: 


$$
\begin{aligned}
w(s)+\int_{\Gamma}\left[\mathrm{q}_{\mathrm{n}}^{*}\right. & \left.(\mathrm{s}, \mathrm{Q}) \mathrm{w}(\mathrm{Q})-\mathrm{m}_{\mathrm{n}}^{*}(\mathrm{~s}, \mathrm{Q}) \frac{\partial w}{\partial n}(\mathrm{Q})-\mathrm{m}_{\mathrm{ns}}^{*}(\mathrm{~s}, \mathrm{Q}) \frac{\partial w}{\partial s}(\mathrm{Q})\right] d \Gamma(\mathrm{Q}) \\
& =\int_{\Gamma}\left[\mathrm{V}_{\mathrm{n}}(\mathrm{Q}) \mathrm{w}^{*}(\mathrm{~s}, \mathrm{Q})-\mathrm{m}_{\mathrm{n}}(\mathrm{Q}) \frac{\partial w^{*}}{\partial n}(\mathrm{~s}, \mathrm{Q})\right] d \Gamma(\mathrm{Q})+\sum_{i=1}^{N_{c}} R_{c_{i}}(Q) w_{c i}^{*}(\mathrm{~s}, \mathrm{Q}) \\
& +\int_{\Omega_{\mathrm{g}}} \mathrm{g}(\mathrm{q}) \mathrm{w}^{*}(\mathrm{~s}, \mathrm{q}) \mathrm{d} \Omega_{\mathrm{g}}(\mathrm{q})
\end{aligned}
$$

Aplicando a derivação à equação (3.32) em relação a direção m, obtémse a equação integral da derivada direcional do deslocamento transversal $\partial w(s) / \partial m$ para um ponto s do domínio da placa:

$$
\begin{gathered}
\frac{\partial w(s)}{\partial m}+\int_{\Gamma}\left[\frac{\partial \mathrm{q}_{\mathrm{n}}^{*}(\mathrm{~s}, \mathrm{Q})}{\partial \mathrm{m}} \mathrm{w}(\mathrm{Q})-\frac{\partial \mathrm{m}_{\mathrm{n}}^{*}(\mathrm{~s}, \mathrm{Q})}{\partial \mathrm{m}} \frac{\partial w}{\partial n}(\mathrm{Q})-\frac{\partial \mathrm{m}_{\mathrm{ns}}^{*}(\mathrm{~s}, \mathrm{Q})}{\partial \mathrm{m}} \frac{\partial w}{\partial \mathrm{s}}(\mathrm{Q})\right] d \Gamma(\mathrm{Q}) \\
=\int_{\Gamma}\left[\mathrm{V}_{\mathrm{n}}(\mathrm{Q}) \frac{\partial \mathrm{w}^{*}(\mathrm{~s}, \mathrm{Q})}{\partial \mathrm{m}}-\mathrm{m}_{\mathrm{n}}(\mathrm{Q}) \frac{\partial}{\partial \mathrm{m}}\left(\frac{\partial w^{*}}{\partial n}(\mathrm{~s}, \mathrm{Q})\right)\right] d \Gamma(\mathrm{Q}) \\
+\sum_{i=1}^{N_{c}} R_{c_{i}}(\mathrm{Q}) \frac{\partial \mathrm{w}_{\mathrm{ci}}^{*}(\mathrm{~s}, \mathrm{Q})}{\partial \mathrm{m}}+\int_{\Omega_{\mathrm{g}}} \mathrm{g}(\mathrm{q}) \frac{\partial \mathrm{w}^{*}(\mathrm{~s}, \mathrm{q})}{\partial \mathrm{m}} \mathrm{d} \Omega_{\mathrm{g}}(\mathrm{q})
\end{gathered}
$$

\subsection{Equação integral para um ponto do contorno}

As equações (3.32) e (3.33) são validas para um ponto s qualquer do domínio da placa, entretanto para a formulação do problema pelo método dos elementos de contorno é necessário obter-se a equação integral para um ponto $S$ do contorno. Para isso, considera-se a placa da Figura 3.3, onde o contorno inicial foi acrescido de um contorno circular $\Gamma_{\xi}$ de raio $\xi$ e $\lambda^{+}$e $\lambda^{-}$são cantos do novo contorno da placa. 
$\overrightarrow{v y}^{x}$

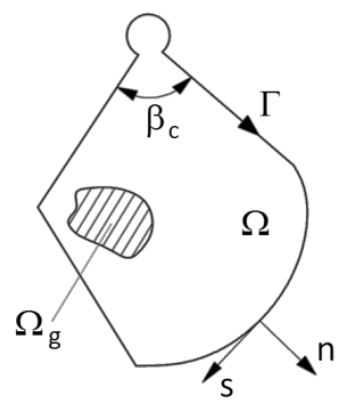

(a)

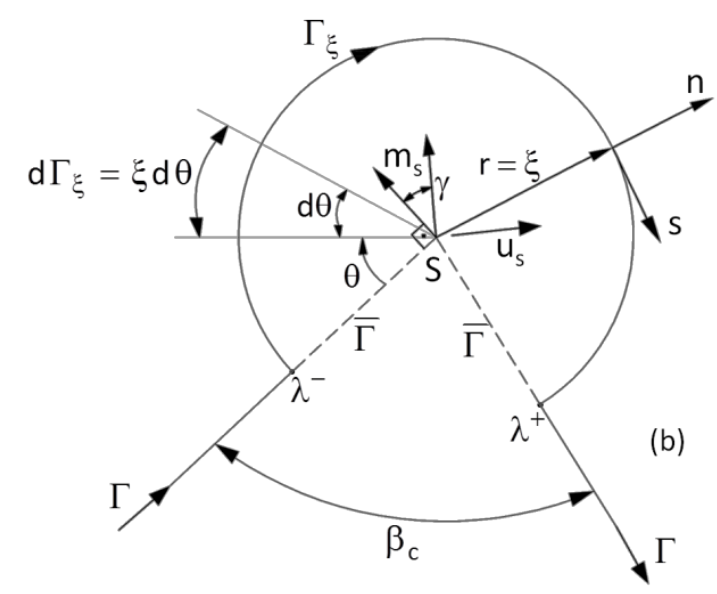

Figura 3.3 - Contorno circular acrescido ao canto da placa.

Com o acréscimo do contorno, a equação (3.32) escrita para o ponto $S$ do contorno da placa resulta:

$$
\begin{aligned}
w(S)+\int_{\Gamma-\bar{\Gamma}}\left[\mathrm{q}_{\mathrm{n}}^{*}(\mathrm{~S}, \mathrm{Q}) \mathrm{w}(\mathrm{Q})-\mathrm{m}_{\mathrm{n}}^{*}(\mathrm{~S}, \mathrm{Q}) \frac{\partial w}{\partial n}(\mathrm{Q})-\mathrm{m}_{\mathrm{ns}}^{*}(\mathrm{~S}, \mathrm{Q}) \frac{\partial w}{\partial s}(Q)\right] d \Gamma(\mathrm{Q}) \\
+\int_{\Gamma_{\xi}}\left[\mathrm{q}_{\mathrm{n}}^{*}(\mathrm{~S}, \mathrm{Q}) \mathrm{w}(\mathrm{Q})-\mathrm{m}_{\mathrm{n}}^{*}(\mathrm{~S}, \mathrm{Q}) \frac{\partial w}{\partial n}(Q)-\mathrm{m}_{\mathrm{ns}}^{*}(\mathrm{~S}, \mathrm{Q}) \frac{\partial w}{\partial s}(Q)\right] d \Gamma_{\xi}(\mathrm{Q}) \\
\quad=\int_{\Gamma-\bar{\Gamma}}\left[\mathrm{V}_{\mathrm{n}}(\mathrm{Q}) \mathrm{w}^{*}(\mathrm{~S}, \mathrm{Q})-\mathrm{m}_{\mathrm{n}}(\mathrm{Q}) \frac{\partial w^{*}}{\partial n}(\mathrm{~S}, \mathrm{Q})\right] d \Gamma(\mathrm{Q}) \\
+\int_{\Gamma_{\xi}}\left[\mathrm{V}_{\mathrm{n}}(\mathrm{Q}) \mathrm{w}^{*}(\mathrm{~S}, \mathrm{Q})-\mathrm{m}_{\mathrm{n}}(\mathrm{Q}) \frac{\partial w^{*}}{\partial n}(\mathrm{~S}, \mathrm{Q})\right] d \Gamma_{\xi}(\mathrm{Q}) \\
+\sum_{i=1}^{N_{c}} R_{c_{i}}(\mathrm{Q}) w_{c i}^{*}(\mathrm{~S}, \mathrm{Q})+R_{c-}(Q) w_{c-}^{*}(\mathrm{~S}, \mathrm{Q})+R_{c+}(Q) w_{c+}^{*}(\mathrm{~S}, \mathrm{Q}) \\
+\int_{\Omega_{\mathrm{g}}} \mathrm{g}(\mathrm{q}) \mathrm{w}^{*}(\mathrm{~S}, \mathrm{q}) \mathrm{d} \Omega_{\mathrm{g}}(\mathrm{q})
\end{aligned}
$$

A medida que $\xi$ se aproxima de zero, o ponto $S$ se aproxima do contorno, e na condição limite, tem-se: 


$$
\begin{aligned}
w(S)+\lim _{\Gamma \rightarrow 0} \int_{\Gamma-\bar{\Gamma}} & {\left[\mathrm{q}_{\mathrm{n}}^{*}(\mathrm{~S}, \mathrm{Q}) \mathrm{w}(\mathrm{Q})-\mathrm{m}_{\mathrm{n}}^{*}(\mathrm{~S}, \mathrm{Q}) \frac{\partial w}{\partial n}(\mathrm{Q})-\mathrm{m}_{\mathrm{ns}}^{*}(\mathrm{~S}, \mathrm{Q}) \frac{\partial w}{\partial s}(\mathrm{Q})\right] d \Gamma(\mathrm{Q}) } \\
& +\lim _{\xi \rightarrow 0} \int_{\Gamma_{\xi}}\left[\mathrm{q}_{\mathrm{n}}^{*}(\mathrm{~S}, \mathrm{Q}) \mathrm{w}(\mathrm{Q})-\mathrm{m}_{\mathrm{n}}^{*}(\mathrm{~S}, \mathrm{Q}) \frac{\partial w}{\partial n}(\mathrm{Q})-\mathrm{m}_{\mathrm{ns}}^{*}(\mathrm{~S}, \mathrm{Q}) \frac{\partial w}{\partial s}(\mathrm{Q})\right] d \Gamma_{\xi}(\mathrm{Q}) \\
& =\lim _{\Gamma \rightarrow 0} \int_{\Gamma-\bar{\Gamma}}\left[\mathrm{V}_{\mathrm{n}}(\mathrm{Q}) \mathrm{w}^{*}(\mathrm{~S}, \mathrm{Q})-\mathrm{m}_{\mathrm{n}}(\mathrm{Q}) \frac{\partial w^{*}}{\partial n}(\mathrm{~S}, \mathrm{Q})\right] d \Gamma(\mathrm{Q}) \\
& +\lim _{\xi \rightarrow 0} \int_{\Gamma_{\xi}}\left[\mathrm{V}_{\mathrm{n}}(\mathrm{Q}) \mathrm{w}^{*}(\mathrm{~S}, \mathrm{Q})-\mathrm{m}_{\mathrm{n}}(\mathrm{Q}) \frac{\partial w^{*}}{\partial n}(\mathrm{~S}, \mathrm{Q})\right] d \Gamma_{\xi}(\mathrm{Q}) \\
& +\sum_{i=1}^{N_{c}} R_{c_{i}}(Q) w_{c i}^{*}(\mathrm{~S}, \mathrm{Q})+\lim _{\xi \rightarrow 0}\left[R_{c \lambda-}(Q) w_{c \lambda-}^{*}(\mathrm{~S}, \mathrm{Q})+R_{c \lambda+}(Q) w_{c \lambda+}^{*}(\mathrm{~S}, \mathrm{Q})\right] \\
& +\int_{\Omega_{\mathrm{g}}} \mathrm{g}(\mathrm{q}) \mathrm{w}^{*}(\mathrm{~S}, \mathrm{q}) \mathrm{d} \Omega_{\mathrm{g}}(\mathrm{q})
\end{aligned}
$$

Pode-se observar, por definição, o valor principal de CAUCHY representado pelos limites das integrais sobre $\Gamma-\bar{\Gamma}$ indicadas na equação (3.35), conforme PAIVA(1989). Assim:

$$
\begin{gathered}
\lim _{\Gamma \rightarrow 0} \int_{\Gamma-\bar{\Gamma}}\left[\mathrm{q}_{\mathrm{n}}^{*}(\mathrm{~S}, \mathrm{Q}) \mathrm{w}(\mathrm{Q})-\mathrm{m}_{\mathrm{n}}^{*}(\mathrm{~S}, \mathrm{Q}) \frac{\partial w}{\partial n}(\mathrm{Q})-\mathrm{m}_{\mathrm{ns}}^{*}(\mathrm{~S}, \mathrm{Q}) \frac{\partial w}{\partial s}(\mathrm{Q})\right] d \Gamma(\mathrm{Q}) \\
=\int_{\Gamma}\left[\mathrm{q}_{\mathrm{n}}^{*}(\mathrm{~S}, \mathrm{Q}) \mathrm{w}(\mathrm{Q})-\mathrm{m}_{\mathrm{n}}^{*}(\mathrm{~S}, \mathrm{Q}) \frac{\partial w}{\partial n}(\mathrm{Q})-\mathrm{m}_{\mathrm{ns}}^{*}(\mathrm{~S}, \mathrm{Q}) \frac{\partial w}{\partial s}(\mathrm{Q})\right] d \Gamma(\mathrm{Q}) \\
\mathrm{e} \\
\lim _{\bar{\Gamma} \rightarrow 0} \int_{\Gamma-\bar{\Gamma}}\left[\mathrm{V}_{\mathrm{n}}(\mathrm{Q}) \mathrm{w}^{*}(\mathrm{~S}, \mathrm{Q})-\mathrm{m}_{\mathrm{n}}(\mathrm{Q}) \frac{\partial w^{*}}{\partial n}(\mathrm{~S}, \mathrm{Q})\right] d \Gamma(\mathrm{Q}) \\
=\int_{\Gamma}\left[\mathrm{V}_{\mathrm{n}}(\mathrm{Q}) \mathrm{w}^{*}(\mathrm{~S}, \mathrm{Q})-\mathrm{m}_{\mathrm{n}}(\mathrm{Q}) \frac{\partial w^{*}}{\partial n}(\mathrm{~S}, \mathrm{Q})\right] d \Gamma(\mathrm{Q})
\end{gathered}
$$

Estudando agora a parcela de (3.35), referente a integral sobre o contorno acrescido $\Gamma_{\xi}$, pode-se escrever: 
$\lim _{\xi \rightarrow 0} \int_{\Gamma_{\xi}} \phi(\xi) d \Gamma_{\xi}=\lim _{\xi \rightarrow 0} \int_{\Gamma_{\xi}}\left[\mathrm{q}_{\mathrm{n}}^{*}(\mathrm{~S}, \mathrm{Q}) \mathrm{w}(\mathrm{Q})-\mathrm{m}_{\mathrm{n}}^{*}(\mathrm{~S}, \mathrm{Q}) \frac{\partial w}{\partial n}(Q)-\mathrm{m}_{\mathrm{ns}}^{*}(\mathrm{~S}, \mathrm{Q}) \frac{\partial w}{\partial s}(Q)\right] d \Gamma_{\xi}$

Tomando-se por Hölder, segundo PAIVA(1987), tem-se:

$$
\begin{gathered}
|\mathrm{w}(\mathrm{Q})-\mathrm{w}(\mathrm{S})| \leq \mathrm{c}_{1} \mathrm{r}^{\alpha 1}(\mathrm{~S}, \mathrm{Q}) \\
|\partial \mathrm{w}(\mathrm{Q}) / \partial n-\partial \mathrm{w}(\mathrm{S}) / \partial n| \leq \mathrm{c}_{2} \mathrm{r}^{\alpha 2}(\mathrm{~S}, \mathrm{Q}) \\
|\partial \mathrm{w}(\mathrm{Q}) / \partial s-\partial \mathrm{w}(\mathrm{S}) / \partial s| \leq \mathrm{c}_{3} \mathrm{r}^{\alpha 3}(\mathrm{~S}, \mathrm{Q}) \\
0<\alpha_{\mathrm{i}}<1, \quad i=1,2,3
\end{gathered}
$$

Onde, $c_{1}, c_{2}$ e $c_{3}$ são constantes.

Escreve-se então a integral da equação (3.38) como:

$$
\begin{aligned}
\lim _{\xi \rightarrow 0} \int_{\Gamma_{\xi}} \phi(\xi) d \Gamma_{\xi} & =\lim _{\xi \rightarrow 0} \int_{\Gamma_{\xi}}\left[\mathrm{q}_{\mathrm{n}}^{*}(\mathrm{~S}, \mathrm{Q})[\mathrm{w}(\mathrm{Q})-\mathrm{w}(\mathrm{S})]-\mathrm{m}_{\mathrm{n}}^{*}(\mathrm{~S}, \mathrm{Q})\left[\frac{\partial w}{\partial n}(Q)-\frac{\partial w}{\partial n}(S)\right]\right. \\
& \left.-\mathrm{m}_{\mathrm{ns}}^{*}(\mathrm{~S}, \mathrm{Q})\left[\frac{\partial w}{\partial s}(Q)-\frac{\partial w}{\partial s}(S)\right]\right] d \Gamma_{\xi} \\
& +\lim _{\xi \rightarrow 0} \int_{\Gamma_{\xi}} \mathrm{q}_{\mathrm{n}}^{*}(\mathrm{~S}, \mathrm{Q}) \mathrm{w}(\mathrm{S}) d \Gamma_{\xi}+\lim _{\xi \rightarrow 0} \int_{\Gamma_{\xi}} \mathrm{m}_{\mathrm{n}}^{*}(\mathrm{~S}, \mathrm{Q}) \frac{\partial w}{\partial n}(S) d \Gamma_{\xi} \\
& +\lim _{\xi \rightarrow 0} \int_{\Gamma_{\xi}} \mathrm{m}_{\mathrm{ns}}^{*}(\mathrm{~S}, \mathrm{Q}) \frac{\partial w}{\partial s}(S) d \Gamma_{\xi}
\end{aligned}
$$

Onde a primeira integral do lado direito desaparecerá quando $\xi \rightarrow 0$.

Então a integral sobre o contorno $\Gamma_{\xi}$ torna-se:

$$
\lim _{\xi \rightarrow 0} \int_{\Gamma_{\xi}} \phi(\xi) d \Gamma_{\xi}=\lim _{\xi \rightarrow 0} \int_{\Gamma_{\xi}}\left[\mathrm{q}_{\mathrm{n}}^{*}(\mathrm{~S}, \mathrm{Q}) \mathrm{w}(\mathrm{S})-\mathrm{m}_{\mathrm{n}}^{*}(\mathrm{~S}, \mathrm{Q}) \frac{\partial w}{\partial n}(S)-\mathrm{m}_{\mathrm{ns}}^{*}(\mathrm{~S}, \mathrm{Q}) \frac{\partial w}{\partial s}(S)\right] d \Gamma_{\xi}
$$

Retomando a Figura 3.3, é possível extrair: 


$$
\begin{aligned}
& \frac{\partial \mathrm{w}}{\partial \mathrm{n}}(\mathrm{S})=\frac{\partial \mathrm{w}}{\partial \mathrm{m}}(\mathrm{S}) \operatorname{sen}(\theta-\gamma)-\frac{\partial \mathrm{w}}{\partial \mathrm{u}}(\mathrm{S}) \cos (\theta-\gamma) \\
& \frac{\partial \mathrm{w}}{\partial \mathrm{n}}(\mathrm{S})=\frac{\partial \mathrm{w}}{\partial \mathrm{m}}(\mathrm{S}) \cos (\theta-\gamma)+\frac{\partial \mathrm{w}}{\partial \mathrm{u}}(\mathrm{S}) \operatorname{sen}(\theta-\gamma)
\end{aligned}
$$

Sendo: $\theta$ ângulo entre os vetores $\mathbf{n}$ e $\mathbf{r}$, e $\gamma$ ângulo entre os vetores $\mathbf{m}$ e $\mathbf{n}$.

Aplicando agora as expressões (2.117), (2.118), (2.119), (2.120), (2.121), (3.42) e (3.43) na equação (3.41):

$$
\begin{aligned}
\lim _{\xi \rightarrow 0} \int_{\Gamma_{\xi}} \phi(\xi) d \Gamma_{\xi} & =\lim _{\xi \rightarrow 0} \int_{\Gamma_{\xi}}\left[-\frac{1}{2 \pi \xi}(\overrightarrow{\mathrm{n}} \cdot \overrightarrow{\mathrm{r}})\right] w(S) d \Gamma_{\xi} \\
& +\lim _{\xi \rightarrow 0} \int_{\Gamma_{\xi}}\left\{-\frac{1}{4 \pi}\left[(1+\mathrm{v}) \ln \xi+(1-\mathrm{v})(\overrightarrow{\mathrm{n}} \cdot \overrightarrow{\mathrm{r}})^{2}\right.\right. \\
& \left.+\mathrm{v}]\left[\frac{\partial \mathrm{w}}{\partial \mathrm{m}}(\mathrm{S}) \operatorname{sen}(\theta-\gamma)-\frac{\partial \mathrm{w}}{\partial \mathrm{u}}(\mathrm{S}) \cos (\theta-\gamma)\right]\right\} d \Gamma_{\xi} \\
& +\lim _{\xi \rightarrow 0} \int_{\Gamma_{\xi}}\left\{-\frac{(1-\mathrm{v})}{4 \pi}(\overrightarrow{\mathrm{n}} \cdot \overrightarrow{\mathrm{r}})(\overrightarrow{\mathrm{s}} \cdot \overrightarrow{\mathrm{r}})\left[\frac{\partial \mathrm{w}}{\partial \mathrm{m}}(\mathrm{S}) \cos (\theta-\gamma)\right.\right. \\
& \left.\left.+\frac{\partial \mathrm{w}}{\partial \mathrm{u}}(\mathrm{S}) \operatorname{sen}(\theta-\gamma)\right]\right\} d \Gamma_{\xi}
\end{aligned}
$$

Novamente da Figura 3.3, tem-se:

$$
\begin{aligned}
& (\vec{n} . \vec{r})=1 \\
& (\vec{s} . \vec{r})=0 \\
& d \Gamma_{\xi}=\xi \mathrm{d} \theta
\end{aligned}
$$

E aplicando (3.45), (3.46) e (3.47) na equação (3.44), tem-se: 


$$
\begin{aligned}
\lim _{\xi \rightarrow 0} \int_{\Gamma_{\xi}} \phi(\xi) d \Gamma_{\xi} & =\lim _{\xi \rightarrow 0} w(S) \int_{0}^{2 \pi-\beta_{c}}\left[-\frac{1}{2 \pi \xi}\right] \xi \mathrm{d} \theta \\
& +\lim _{\xi \rightarrow 0} \int_{0}^{2 \pi-\beta_{c}}\left\{-\frac{1}{4 \pi}[(1+\mathrm{v}) \ln \xi+1]\left[\frac{\partial \mathrm{w}}{\partial \mathrm{m}}(\mathrm{S}) \operatorname{sen}(\theta-\gamma)\right.\right. \\
& \left.\left.-\frac{\partial \mathrm{w}}{\partial \mathrm{u}}(\mathrm{S}) \cos (\theta-\gamma)\right]\right\} \xi \mathrm{d} \theta
\end{aligned}
$$

e

$$
\lim _{\xi \rightarrow 0} \int_{\Gamma_{\xi}}\left[-\frac{1}{2 \pi \xi}(\vec{n} \cdot \vec{r})\right] w(S) d \Gamma_{\xi}=-\frac{\left(2 \pi-\beta_{c}\right)}{2 \pi} w(S)
$$

Deve-se notar que os demais limites em $\xi$, incluindo parcelas de reações de canto, anulam-se, de onde a equação (3.35) resulta:

$$
\begin{aligned}
K(S) w(S)+\int_{\Gamma} & {\left[\mathrm{q}_{\mathrm{n}}^{*}(\mathrm{~S}, \mathrm{Q}) \mathrm{w}(\mathrm{Q})-\mathrm{m}_{\mathrm{n}}^{*}(\mathrm{~S}, \mathrm{Q}) \frac{\partial w}{\partial n}(\mathrm{Q})-\mathrm{m}_{\mathrm{ns}}^{*}(\mathrm{~S}, \mathrm{Q}) \frac{\partial w}{\partial s}(\mathrm{Q})\right] d \Gamma(\mathrm{Q}) } \\
& =\int_{\Gamma}\left[\mathrm{V}_{\mathrm{n}}(\mathrm{Q}) \mathrm{w}^{*}(\mathrm{~S}, \mathrm{Q})-\mathrm{m}_{\mathrm{n}}(\mathrm{Q}) \frac{\partial w^{*}}{\partial n}(\mathrm{~S}, \mathrm{Q})\right] d \Gamma(\mathrm{Q})+\sum_{i=1}^{N_{c}} R_{c_{i}}(Q) w_{c i}^{*}(\mathrm{~S}, \mathrm{Q}) \\
& +\int_{\Omega_{\mathrm{g}}} \mathrm{g}(\mathrm{q}) \mathrm{w}^{*}(\mathrm{~S}, \mathrm{q}) \mathrm{d} \Omega_{\mathrm{g}}(\mathrm{q})
\end{aligned}
$$

Com,

$$
K(S)=\frac{\beta_{c}}{2 \pi}
$$

Para um ponto $S$ que não pertença a um canto da placa a constante $K(S)$ resulta:

$$
K(S)=1 / 2
$$


Escrevendo agora a equação integral (3.33) da derivada direcional $\partial w(S) / \partial m$ para o ponto $S$ da Figura 3.3, tem-se:

$$
\begin{aligned}
\frac{\partial w(S)}{\partial m}+\int_{\Gamma-\bar{\Gamma}}[ & \left.\frac{\partial \mathrm{q}_{\mathrm{n}}^{*}(\mathrm{~S}, \mathrm{Q})}{\partial \mathrm{m}} \mathrm{w}(\mathrm{Q})-\frac{\partial \mathrm{m}_{\mathrm{n}}^{*}(\mathrm{~S}, \mathrm{Q})}{\partial \mathrm{m}} \frac{\partial w}{\partial n}(\mathrm{Q})-\frac{\partial \mathrm{m}_{\mathrm{ns}}^{*}(\mathrm{~S}, \mathrm{Q})}{\partial \mathrm{m}} \frac{\partial w}{\partial s}(\mathrm{Q})\right] d \Gamma(\mathrm{Q}) \\
& +\int_{\Gamma_{\xi}}\left[\frac{\partial \mathrm{q}_{\mathrm{n}}^{*}(\mathrm{~S}, \mathrm{Q})}{\partial \mathrm{m}} \mathrm{w}(\mathrm{Q})-\frac{\partial \mathrm{m}_{\mathrm{n}}^{*}(\mathrm{~S}, \mathrm{Q})}{\partial \mathrm{m}} \frac{\partial w}{\partial n}(\mathrm{Q})\right. \\
& \left.-\frac{\partial \mathrm{m}_{\mathrm{n} S}^{*}(\mathrm{~S}, \mathrm{Q})}{\partial \mathrm{m}} \frac{\partial w}{\partial s}(\mathrm{Q})\right] d \Gamma_{\xi}(\mathrm{Q}) \\
& =\int_{\Gamma-\bar{\Gamma}}\left[\mathrm{V}_{\mathrm{n}}(\mathrm{Q}) \frac{\partial \mathrm{w}^{*}(\mathrm{~S}, \mathrm{Q})}{\partial \mathrm{m}}-\mathrm{m}_{\mathrm{n}}(\mathrm{Q}) \frac{\partial}{\partial \mathrm{m}}\left(\frac{\partial w^{*}}{\partial n}(\mathrm{~S}, \mathrm{Q})\right)\right] d \Gamma(\mathrm{Q}) \\
& +\int_{\Gamma_{\xi}}\left[\mathrm{V}_{\mathrm{n}}(\mathrm{Q}) \frac{\partial \mathrm{w}^{*}(\mathrm{~S}, \mathrm{Q})}{\partial \mathrm{m}}-\mathrm{m}_{\mathrm{n}}(\mathrm{Q}) \frac{\partial}{\partial \mathrm{m}}\left(\frac{\partial w^{*}}{\partial n}(\mathrm{~S}, \mathrm{Q})\right)\right] d \Gamma_{\xi}(\mathrm{Q}) \\
& +\sum_{i=1}^{N_{c}} R_{c_{i}}(Q) \frac{\partial \mathrm{w}_{\mathrm{ci}}^{*}(\mathrm{~S}, \mathrm{Q})}{\partial \mathrm{m}} \\
& +\left[R_{c \lambda-}(Q) \frac{\partial \mathrm{w}_{c \lambda-}^{*}(\mathrm{~S}, \mathrm{Q})}{\partial \mathrm{m}}+R_{c \lambda+}(Q) \frac{\partial \mathrm{w}_{c \lambda+}^{*}(\mathrm{~S}, \mathrm{Q})}{\partial \mathrm{m}}\right] \\
& +\int_{\Omega_{\mathrm{g}}} \mathrm{g}(\mathrm{q}) \frac{\partial \mathrm{w}^{*}(\mathrm{~S}, \mathrm{q})}{\partial \mathrm{m}} \mathrm{d} \Omega_{\mathrm{g}}(\mathrm{q})
\end{aligned}
$$

E para $\xi$ tendendo a zero, o ponto S pertence ao contorno. Assim, obtêmse para equação anterior (3.53): 


$$
\begin{aligned}
\frac{\partial w(S)}{\partial m}+\lim _{\Gamma \rightarrow 0} \int_{\Gamma-\bar{\Gamma}} & {\left[\frac{\partial \mathrm{q}_{\mathrm{n}}^{*}(\mathrm{~S}, \mathrm{Q})}{\partial \mathrm{m}} \mathrm{w}(\mathrm{Q})-\frac{\partial \mathrm{m}_{\mathrm{n}}^{*}(\mathrm{~S}, \mathrm{Q})}{\partial \mathrm{m}} \frac{\partial w}{\partial n}(\mathrm{Q})-\frac{\partial \mathrm{m}_{\mathrm{ns}}^{*}(\mathrm{~S}, \mathrm{Q})}{\partial \mathrm{m}} \frac{\partial w}{\partial \mathrm{s}}(\mathrm{Q})\right] d \Gamma(\mathrm{Q}) } \\
& +\lim _{\xi \rightarrow 0} \int_{\Gamma_{\xi}}\left[\frac{\partial \mathrm{q}_{\mathrm{n}}^{*}(\mathrm{~S}, \mathrm{Q})}{\partial \mathrm{m}} \mathrm{w}(\mathrm{Q})-\frac{\partial \mathrm{m}_{\mathrm{n}}^{*}(\mathrm{~S}, \mathrm{Q})}{\partial \mathrm{m}} \frac{\partial w}{\partial n}(\mathrm{Q})\right. \\
& \left.-\frac{\partial \mathrm{m}_{\mathrm{ns}}^{*}(\mathrm{~S}, \mathrm{Q})}{\partial \mathrm{m}} \frac{\partial w}{\partial \mathrm{s}}(\mathrm{Q})\right] d \Gamma_{\xi}(\mathrm{Q}) \\
& =\lim _{\bar{\Gamma} \rightarrow 0} \int_{\Gamma-\bar{\Gamma}}\left[\mathrm{V}_{\mathrm{n}}(\mathrm{Q}) \frac{\partial \mathrm{w}^{*}(\mathrm{~S}, \mathrm{Q})}{\partial \mathrm{m}}-\mathrm{m}_{\mathrm{n}}(\mathrm{Q}) \frac{\partial}{\partial \mathrm{m}}\left(\frac{\partial w^{*}}{\partial n}(\mathrm{~S}, \mathrm{Q})\right)\right] d \Gamma(\mathrm{Q}) \\
& +\lim _{\xi \rightarrow 0} \int_{\Gamma_{\xi}}\left[\mathrm{V}_{\mathrm{n}}(\mathrm{Q}) \frac{\partial \mathrm{w}^{*}(\mathrm{~S}, \mathrm{Q})}{\partial \mathrm{m}}-\mathrm{m}_{\mathrm{n}}(\mathrm{Q}) \frac{\partial}{\partial \mathrm{m}}\left(\frac{\partial w^{*}}{\partial n}(\mathrm{~S}, \mathrm{Q})\right)\right] d \Gamma_{\xi}(\mathrm{Q}) \\
& +\sum_{i=1}^{N_{c}} R_{c_{i}}(Q) \frac{\partial \mathrm{w}_{\mathrm{ci}}^{*}(\mathrm{~S}, \mathrm{Q})}{\partial \mathrm{m}} \\
& +\lim _{\xi \rightarrow 0}\left[R_{c \lambda-}(Q) \frac{\partial \mathrm{w}_{c \lambda-}^{*}(\mathrm{~S}, \mathrm{Q})}{\partial \mathrm{m}}+R_{c \lambda+}(\mathrm{Q}) \frac{\partial \mathrm{w}_{c \lambda+}^{*}(\mathrm{~S}, \mathrm{Q})}{\partial \mathrm{m}}\right] \\
& +\int_{\Omega_{\mathrm{g}}} \mathrm{g}(\mathrm{q}) \frac{\partial \mathrm{w}^{*}(\mathrm{~S}, \mathrm{q})}{\partial \mathrm{m}} \mathrm{d} \Omega_{\mathrm{g}}(\mathrm{q})
\end{aligned}
$$

Os limites das integrais sobre $\Gamma-\bar{\Gamma}$ são os valores principais das integrais sobre $Г$. De acordo com OLIVEIRA (1998), realizando um deslocamento vertical de corpo rígido com a placa igual a $-w(S)$, podem-se eliminar as singularidades que aparecem na equação (3.54) quando $\xi \rightarrow 0$. Resultando: 


$$
\begin{aligned}
& \frac{\partial w(S)}{\partial m}+\int_{\Gamma}\left[\frac{\partial \mathrm{q}_{\mathrm{n}}^{*}(\mathrm{~S}, \mathrm{Q})}{\partial \mathrm{m}}[\mathrm{w}(\mathrm{Q})-\mathrm{w}(\mathrm{S})]-\frac{\partial \mathrm{m}_{\mathrm{n}}^{*}(\mathrm{~S}, \mathrm{Q})}{\partial \mathrm{m}} \frac{\partial w}{\partial n}(\mathrm{Q})\right. \\
&\left.\quad-\frac{\partial \mathrm{m}_{\mathrm{ns}}^{*}(\mathrm{~S}, \mathrm{Q})}{\partial \mathrm{m}} \frac{\partial w}{\partial \mathrm{s}}(\mathrm{Q})\right] d \Gamma(\mathrm{Q}) \\
& \quad+\lim _{\xi \rightarrow 0} \int_{\Gamma_{\xi}}\left[\frac{\partial \mathrm{q}_{\mathrm{n}}^{*}(\mathrm{~S}, \mathrm{Q})}{\partial \mathrm{m}}[\mathrm{w}(\mathrm{Q})-\mathrm{w}(\mathrm{S})]-\frac{\partial \mathrm{m}_{\mathrm{n}}^{*}(\mathrm{~S}, \mathrm{Q})}{\partial \mathrm{m}} \frac{\partial w}{\partial n}(\mathrm{Q})\right. \\
&\left.-\frac{\partial \mathrm{m}_{\mathrm{ns}}^{*}(\mathrm{~S}, \mathrm{Q})}{\partial \mathrm{m}} \frac{\partial \mathrm{w}}{\partial s}(\mathrm{Q})\right] d \Gamma_{\xi}(\mathrm{Q}) \\
&=\int_{\Gamma}\left[\mathrm{V}_{\mathrm{n}}(\mathrm{Q}) \frac{\partial \mathrm{w}^{*}(\mathrm{~S}, \mathrm{Q})}{\partial \mathrm{m}}-\mathrm{m}_{\mathrm{n}}(\mathrm{Q}) \frac{\partial}{\partial \mathrm{m}}\left(\frac{\partial w^{*}}{\partial n}(\mathrm{~S}, \mathrm{Q})\right)\right] d \Gamma(\mathrm{Q}) \\
&+\lim _{\xi \rightarrow 0} \int_{\Gamma_{\xi}}\left[\mathrm{V}_{\mathrm{n}}(\mathrm{Q}) \frac{\partial \mathrm{w}^{*}(\mathrm{~S}, \mathrm{Q})}{\partial \mathrm{m}}-\mathrm{m}_{\mathrm{n}}(\mathrm{Q}) \frac{\partial}{\partial \mathrm{m}}\left(\frac{\partial w^{*}}{\partial n}(\mathrm{~S}, \mathrm{Q})\right)\right] d \Gamma_{\xi}(\mathrm{Q}) \\
&+\sum_{i=1}^{N_{c}} R_{c_{i}}(Q) \frac{\partial \mathrm{w}_{\mathrm{ci}}^{*}(\mathrm{~S}, \mathrm{Q})}{\partial \mathrm{m}} \\
&+\lim _{\xi \rightarrow 0}\left[R_{c \lambda-}(Q) \frac{\partial \mathrm{w}_{c \lambda-}^{*}(\mathrm{~S}, \mathrm{Q})}{\partial \mathrm{m}}+R_{c \lambda+}(\mathrm{g}) \frac{\partial \mathrm{w}_{c \lambda+}^{*}(\mathrm{~S}, \mathrm{Q})}{\partial \mathrm{m}}\right] \\
& \frac{\partial \mathrm{w}^{*}(\mathrm{~S}, \mathrm{q})}{\partial \mathrm{m}} \mathrm{d} \Omega_{\mathrm{g}}(\mathrm{q})
\end{aligned}
$$

Assim, escreve-se a integral sobre $\Gamma_{\xi}$ como:

$$
\begin{aligned}
\lim _{\xi \rightarrow 0} \int_{\Gamma_{\xi}} \phi(\xi) d \Gamma_{\xi} & =\lim _{\xi \rightarrow 0} \int_{\Gamma_{\xi}}\left[\frac{\partial \mathrm{q}_{\mathrm{n}}^{*}(\mathrm{~S}, \mathrm{Q})}{\partial \mathrm{m}}[\mathrm{w}(\mathrm{Q})-\mathrm{w}(\mathrm{S})]-\frac{\partial \mathrm{m}_{\mathrm{n}}^{*}(\mathrm{~S}, \mathrm{Q})}{\partial \mathrm{m}}\left[\frac{\partial w}{\partial n}(Q)-\frac{\partial w}{\partial n}(S)\right]\right. \\
& \left.-\frac{\partial \mathrm{m}_{\mathrm{ns}}^{*}(\mathrm{~S}, \mathrm{Q})}{\partial \mathrm{m}}\left[\frac{\partial w}{\partial s}(Q)-\frac{\partial w}{\partial s}(S)\right]\right] d \Gamma_{\xi}-\lim _{\xi \rightarrow 0} \int_{\Gamma_{\xi}} \frac{\partial \mathrm{m}_{\mathrm{n}}^{*}(\mathrm{~S}, \mathrm{Q})}{\partial \mathrm{m}} \frac{\partial w}{\partial n}(S) d \Gamma_{\xi} \\
& -\lim _{\xi \rightarrow 0} \int_{\Gamma_{\xi}} \frac{\partial \mathrm{m}_{\mathrm{ns}}^{*}(\mathrm{~S}, \mathrm{Q})}{\partial \mathrm{m}} \frac{\partial w}{\partial s}(S) d \Gamma_{\xi}
\end{aligned}
$$


Tomando as condições de Hölder, segundo PAIVA(1987), tem-se:

$$
\begin{aligned}
\lim _{\xi \rightarrow 0} \int_{\Gamma_{\xi}} \phi(\xi) d \Gamma_{\xi} & =\lim _{\xi \rightarrow 0} \int_{\Gamma_{\xi}} \frac{\partial \mathrm{q}_{\mathrm{n}}^{*}(\mathrm{~S}, \mathrm{Q})}{\partial \mathrm{m}}[\mathrm{w}(\mathrm{Q})-\mathrm{w}(\mathrm{S})] d \Gamma_{\xi}-\lim _{\xi \rightarrow 0} \int_{\Gamma_{\xi}} \frac{\partial \mathrm{m}_{\mathrm{n}}^{*}(\mathrm{~S}, \mathrm{Q})}{\partial \mathrm{m}} \frac{\partial w}{\partial n}(S) d \Gamma_{\xi} \\
& -\lim _{\xi \rightarrow 0} \int_{\Gamma_{\xi}} \frac{\partial \mathrm{m}_{\mathrm{ns}}^{*}(\mathrm{~S}, \mathrm{Q})}{\partial \mathrm{m}} \frac{\partial w}{\partial s}(S) d \Gamma_{\xi}
\end{aligned}
$$

De acordo com a Figura 3.4, que mostra os pontos $S$ e $Q$ e seus deslocamentos, quando $\xi \rightarrow 0$, escreve-se:

$$
w(Q)-w(S)=\xi \frac{\partial w}{\partial n}(S)
$$
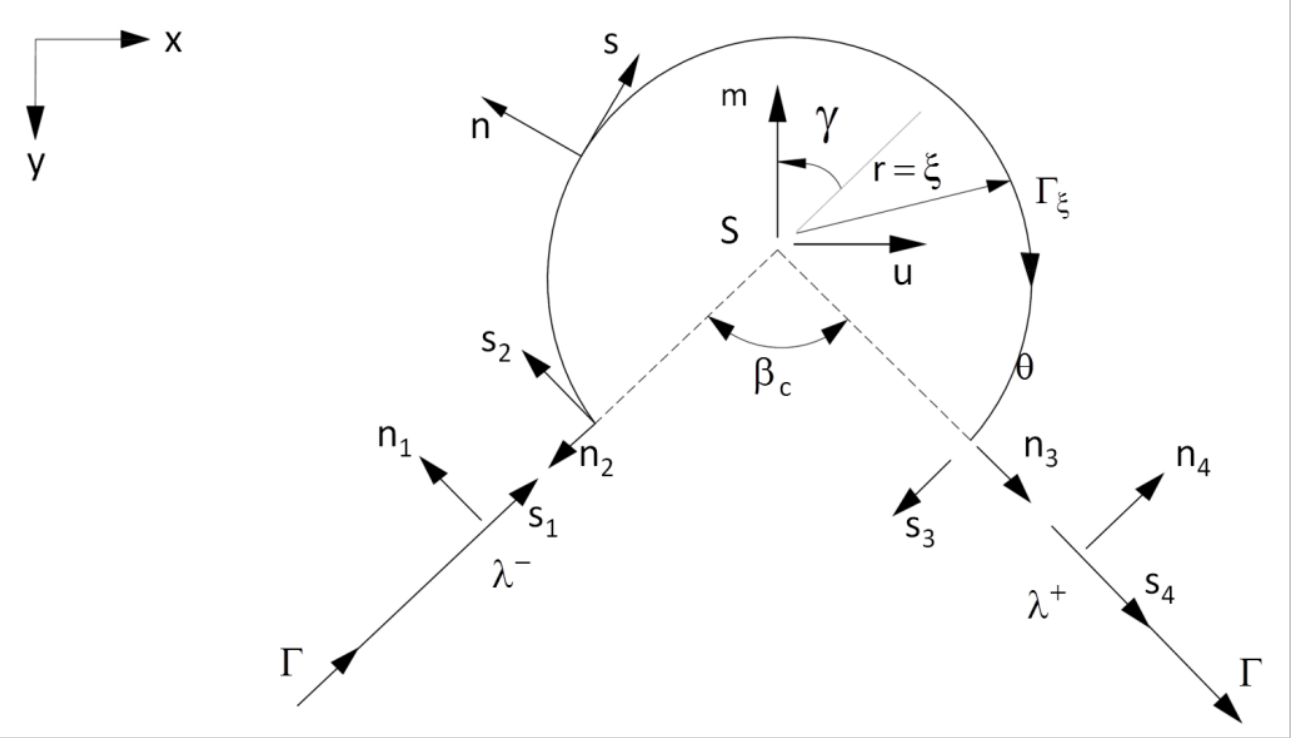

Figura 3.4 - Sistema de coordenadas referentes aos pontos anterior e posterior aos cantos.

Com a equação (3.58) aplicada na equação (3.57), obtém-se: 


$$
\begin{aligned}
\lim _{\xi \rightarrow 0} \int_{\Gamma_{\xi}} \phi(\xi) d \Gamma_{\xi} & =\lim _{\xi \rightarrow 0} \int_{\Gamma_{\xi}} \frac{\partial \mathrm{q}_{\mathrm{n}}^{*}(\mathrm{~S}, \mathrm{Q})}{\partial \mathrm{m}} \xi \frac{\partial w}{\partial n}(S) d \Gamma_{\xi}-\lim _{\xi \rightarrow 0} \int_{\Gamma_{\xi}} \frac{\partial \mathrm{m}_{\mathrm{n}}^{*}(\mathrm{~S}, \mathrm{Q})}{\partial \mathrm{m}} \frac{\partial w}{\partial n}(S) d \Gamma_{\xi} \\
& -\lim _{\xi \rightarrow 0} \int_{\Gamma_{\xi}} \frac{\partial \mathrm{m}_{\mathrm{ns}}^{*}(\mathrm{~S}, \mathrm{Q})}{\partial \mathrm{m}} \frac{\partial w}{\partial s}(S) d \Gamma_{\xi}
\end{aligned}
$$

Com as soluções fundamentais (2.137), (2.138) e (2.139) substituídas na equação (3.59), obtém-se:

$$
\begin{aligned}
\lim _{\xi \rightarrow 0} \int_{\Gamma_{\xi}} \phi(\xi) d \Gamma_{\xi} & =\lim _{\xi \rightarrow 0} \int_{\Gamma_{\xi}}\left\{\frac{1}{2 \pi \xi^{2}}[(\overrightarrow{\mathrm{m}} \cdot \overrightarrow{\mathrm{n}})-2(\overrightarrow{\mathrm{m}} \cdot \overrightarrow{\mathrm{r}})]\right\} \xi \frac{\partial w}{\partial n}(S) d \Gamma_{\xi} \\
& -\lim _{\xi \rightarrow 0} \int_{\Gamma_{\xi}}\left[\frac{1}{4 \pi \xi}(1+\mathrm{v})(\overrightarrow{\mathrm{m}} \cdot \overrightarrow{\mathrm{r}})\right] \frac{\partial w}{\partial n}(S) d \Gamma_{\xi} \\
& -\lim _{\xi \rightarrow 0} \int_{\Gamma_{\xi}}\left[-\frac{(1-\mathrm{v})}{4 \pi \xi}(-\overrightarrow{\mathrm{m}} \cdot \overrightarrow{\mathrm{s}})\right] \frac{\partial w}{\partial s}(S) d \Gamma_{\xi}
\end{aligned}
$$

Os termos referentes a $\partial w(S) / \partial n$ podem ser agrupados, considerando que $(\overrightarrow{\mathrm{m}} \cdot \overrightarrow{\mathrm{n}})=(\overrightarrow{\mathrm{m}} \cdot \overrightarrow{\mathrm{r}})$, portanto, tem-se:

$$
\begin{aligned}
\lim _{\xi \rightarrow 0} \int_{\Gamma_{\xi}} \phi(\xi) d \Gamma_{\xi} & =\lim _{\xi \rightarrow 0} \int_{\Gamma_{\xi}}\left\{\frac{1}{2 \pi \xi^{2}}[-(\overrightarrow{\mathrm{m}} \cdot \overrightarrow{\mathrm{r}})]-\left[\frac{1}{4 \pi \xi}(1+\mathrm{v})(\overrightarrow{\mathrm{m}} \cdot \overrightarrow{\mathrm{r}})\right]\right\} \frac{\partial w}{\partial n}(S) d \Gamma_{\xi} \\
& -\lim _{\xi \rightarrow 0} \int_{\Gamma_{\xi}}\left[\frac{(1-\mathrm{v})}{4 \pi \xi}(\overrightarrow{\mathrm{m}} \cdot \overrightarrow{\mathrm{s}})\right] \frac{\partial w}{\partial s}(S) d \Gamma_{\xi}
\end{aligned}
$$

Ou ainda, 


$$
\begin{aligned}
\lim _{\xi \rightarrow 0} \int_{\Gamma_{\xi}} \phi(\xi) d \Gamma_{\xi} & =\lim _{\xi \rightarrow 0} \int_{\Gamma_{\xi}}\left[-\frac{(3+v)}{4 \pi \xi}(\overrightarrow{\mathrm{m}} \cdot \overrightarrow{\mathrm{r}})\right] \frac{\partial w}{\partial n}(S) d \Gamma_{\xi} \\
& -\lim _{\xi \rightarrow 0} \int_{\Gamma_{\xi}}\left[\frac{(1-\mathrm{v})}{4 \pi \xi}(\overrightarrow{\mathrm{m}} \cdot \overrightarrow{\mathrm{s}})\right] \frac{\partial w}{\partial s}(S) d \Gamma_{\xi}
\end{aligned}
$$

Considerando as expressões (3.42), (3.43), (3.45), e ainda:

$$
\begin{aligned}
& (\overrightarrow{\mathrm{m}} \cdot \overrightarrow{\mathrm{r}})=\operatorname{sen}(\theta-\gamma) \\
& (\overrightarrow{\mathrm{m}} \cdot \overrightarrow{\mathrm{s}})=\cos (\theta-\gamma)
\end{aligned}
$$

Obtém-se:

$$
\begin{aligned}
\lim _{\xi \rightarrow 0} \int_{\Gamma_{\xi}} \phi(\xi) d \Gamma_{\xi} & =\lim _{\xi \rightarrow 0} \int_{0}^{2 \pi-\beta_{c}}\left[-\frac{(3+\mathrm{v})}{4 \pi \xi}\right] \operatorname{sen}(\theta \\
& -\gamma)\left[\frac{\partial \mathrm{w}}{\partial \mathrm{m}}(\mathrm{S}) \operatorname{sen}(\theta-\gamma)-\frac{\partial \mathrm{w}}{\partial \mathrm{u}}(\mathrm{S}) \cos (\theta-\gamma)\right] \xi d \theta \\
& -\lim _{\xi \rightarrow 0} \int_{0}^{2 \pi-\beta_{c}}\left\{\frac { ( 1 - \mathrm { v } ) } { 4 \pi \xi } \operatorname { c o s } ( \theta - \gamma ) \left[\frac{\partial \mathrm{w}}{\partial \mathrm{m}}(\mathrm{S}) \cos (\theta-\gamma)\right.\right. \\
& \left.\left.+\frac{\partial \mathrm{w}}{\partial \mathrm{u}}(\mathrm{S}) \operatorname{sen}(\theta-\gamma)\right]\right\} \xi d \theta
\end{aligned}
$$

Em que, $\gamma$ é o ângulo entre os sistemas de coordenas $(n, s)$ e $(m, u)$, conforme Figura 3.4.

Pode-se ainda rearranjar os termos internos as integrais: 


$$
\begin{aligned}
\lim _{\xi \rightarrow 0} \int_{\Gamma_{\xi}} \phi(\xi) d \Gamma_{\xi} & =\int_{0}^{2 \pi-\beta_{c}}\left[-\frac{(3+\mathrm{v})}{4 \pi}\right]\left[\frac{\partial \mathrm{w}}{\partial \mathrm{m}}(\mathrm{S}) \operatorname{sen}^{2}(\theta-\gamma)\right. \\
& \left.-\frac{\partial \mathrm{w}}{\partial \mathrm{u}}(\mathrm{S}) \operatorname{sen}(\theta-\gamma) \cos (\theta-\gamma)\right] d \theta \\
& -\int_{0}^{2 \pi-\beta_{c}}\left\{\frac { ( 1 - \mathrm { v } ) } { 4 \pi \xi } \left[\frac{\partial \mathrm{w}}{\partial \mathrm{m}}(\mathrm{S}) \cos ^{2}(\theta-\gamma)\right.\right. \\
& \left.\left.+\frac{\partial \mathrm{w}}{\partial \mathrm{u}}(\mathrm{S}) \operatorname{sen}(\theta-\gamma) \cos (\theta-\gamma)\right]\right\} \xi d \theta
\end{aligned}
$$

Ou ainda:

$$
\begin{aligned}
\lim _{\xi \rightarrow 0} \int_{\Gamma_{\xi}} \phi(\xi) d \Gamma_{\xi} & =-\frac{(3+\mathrm{v})}{4 \pi}\left\{\frac{1}{4}\left[4 \pi-2 \beta_{\mathrm{c}}+\operatorname{sen} 2\left(\gamma+\beta_{\mathrm{c}}\right)-\operatorname{sen} 2 \gamma\right] \frac{\partial \mathrm{w}}{\partial \mathrm{m}}(\mathrm{S})\right. \\
& \left.+\frac{1}{4}\left[\cos 2\left(\gamma+\beta_{\mathrm{c}}\right)-\cos 2 \gamma\right] \frac{\partial \mathrm{w}}{\partial \mathrm{u}}(\mathrm{S})\right\} \\
& -\frac{(1-\mathrm{v})}{4 \pi}\left\{\frac{1}{4}\left[4 \pi-2 \beta_{\mathrm{c}}-\operatorname{sen} 2\left(\gamma+\beta_{\mathrm{c}}\right)+\operatorname{sen} 2 \gamma\right] \frac{\partial \mathrm{w}}{\partial \mathrm{m}}(\mathrm{S})\right. \\
& \left.-\frac{1}{4}\left[\cos 2\left(\gamma+\beta_{\mathrm{c}}\right)-\cos 2 \gamma\right] \frac{\partial \mathrm{w}}{\partial \mathrm{u}}(\mathrm{S})\right\}
\end{aligned}
$$

Os termos referentes as derivadas dos deslocamentos transversais podem ser reagrupados, resultando:

$$
\begin{aligned}
\lim _{\xi \rightarrow 0} \int_{\Gamma_{\xi}} \phi(\xi) d \Gamma_{\xi} & =\left\{-\frac{\left(2 \pi-\beta_{\mathrm{c}}\right)}{2 \pi}+\frac{(1+\mathrm{v})}{8 \pi}\left[\operatorname{sen} 2 \gamma-\operatorname{sen} 2\left(\gamma+\beta_{\mathrm{c}}\right)\right] \frac{\partial \mathrm{w}}{\partial \mathrm{m}}(\mathrm{S})\right. \\
& \left.+\frac{(1+\mathrm{v})}{8 \pi}\left[\cos 2 \gamma-\cos 2\left(\gamma+\beta_{\mathrm{c}}\right)\right] \frac{\partial \mathrm{w}}{\partial \mathrm{u}}(\mathrm{S})\right\}
\end{aligned}
$$

Sendo nulos os demais limites dependentes de $\xi$ equação (3.55).

Obtém-se finalmente a expressão da derivada da equação integral em um canto do contorno, feitas as substituições em (3.55): 


$$
\begin{aligned}
K_{1}(S) \frac{\partial w}{\partial m_{s}}(S) & +K_{2}(S) \frac{\partial w}{\partial u_{s}}(S) \\
& +\int_{\Gamma}\left[\frac{\partial \mathrm{q}_{\mathrm{n}}^{*}(\mathrm{~S}, \mathrm{Q})}{\partial m_{s}} \mathrm{w}(\mathrm{Q})-\frac{\partial \mathrm{m}_{\mathrm{n}}^{*}(\mathrm{~S}, \mathrm{Q})}{\partial m_{s}} \frac{\partial w}{\partial n}(\mathrm{Q})-\frac{\partial \mathrm{m}_{\mathrm{ns}}^{*}(\mathrm{~S}, \mathrm{Q})}{\partial m_{s}} \frac{\partial w}{\partial s}(\mathrm{Q})\right] d \Gamma(\mathrm{Q}) \\
& =\int_{\Gamma}\left\{\mathrm{V}_{\mathrm{n}}(\mathrm{Q}) \frac{\partial \mathrm{w}^{*}(\mathrm{~S}, \mathrm{Q})}{\partial m_{s}}-\mathrm{m}_{\mathrm{n}}(\mathrm{Q}) \frac{\partial}{\partial m_{s}}\left[\frac{\partial w^{*}}{\partial n}(\mathrm{~S}, \mathrm{Q})\right]\right\} d \Gamma(\mathrm{Q}) \\
& +\sum_{i=1}^{N_{c}} R_{c_{i}}(Q) \frac{\partial \mathrm{w}_{\mathrm{ci}}^{*}(\mathrm{~S}, \mathrm{Q})}{\partial m_{s}}+\int_{\Omega_{\mathrm{g}}} \mathrm{g}(\mathrm{q}) \frac{\partial \mathrm{w}^{*}(\mathrm{~S}, \mathrm{q})}{\partial m_{s}} \mathrm{~d} \Omega_{\mathrm{g}}(\mathrm{q})
\end{aligned}
$$

Sendo:

$$
\begin{gathered}
K_{1}(S)=\frac{\beta}{2 \pi}+\frac{1+v}{8 \pi}[\operatorname{sen} 2 \gamma-\operatorname{sen} 2(\gamma+\beta)] \\
K_{2}(S)=\frac{1+v}{8 \pi}[\cos 2 \gamma-\cos 2(\gamma+\beta)]
\end{gathered}
$$

Em que, $\gamma$ é o ângulo entre os sistemas de coordenas $(n, s)$ e $(m, u)$, conforme Figura 3.4 .

Sendo a expressão de K(S), dada por:

$$
\begin{aligned}
K(S)=\left\{\frac{\beta_{c}}{2 \pi}+\right. & \left.\frac{(1+\mathrm{v})}{8 \pi}\left[\operatorname{sen} 2 \gamma-\operatorname{sen} 2\left(\gamma+\beta_{\mathrm{c}}\right)\right]\right\} \frac{\partial w}{\partial m}(S) \\
& +\frac{(1+\mathrm{v})}{8 \pi}\left[\cos 2 \gamma-\cos 2\left(\gamma+\beta_{\mathrm{c}}\right)\right] \frac{\partial w}{\partial u}(S) \\
& =K_{1}(S) \frac{\partial w}{\partial m}(S)+K_{2}(S) \frac{\partial w}{\partial u}(S)
\end{aligned}
$$

Para o caso da coordenada m coincidir com a direção normal $n_{1}$, anterior ao canto, e $\gamma=0$, obtém-se: 


$$
K(S)=\left[\frac{\beta_{c}}{2 \pi}-\frac{1+\mathrm{v}}{8 \pi} \operatorname{sen} 2 \beta_{\mathrm{c}}\right] \frac{\partial w}{\partial n}(S)+\frac{1+\mathrm{v}}{8 \pi}\left[1-\cos 2 \beta_{\mathrm{c}}\right] \frac{\partial w}{\partial s}(S)
$$

e para $m=n_{1}$, anterior ao canto, e $\gamma=\pi / 2$ :

$$
K(S)=\left[\frac{\beta_{c}}{2 \pi}+\frac{1+\mathrm{v}}{8 \pi} \operatorname{sen} 2 \beta_{\mathrm{c}}\right] \frac{\partial w}{\partial n}(S)-\frac{1+\mathrm{v}}{8 \pi}\left[1-\cos 2 \beta_{\mathrm{c}}\right] \frac{\partial w}{\partial s}(S)
$$

\subsection{Transformações das integrais de domínio}

As integrais de domínio das equações (3.32), (3.33), (3.50) e (3.69) serão transformadas em integrais sobre o contorno da região carregada $\Omega_{\mathrm{g}}$ (Figura 3.5) baseado no exposto em PAIVA(1987).



Figura 3.5 - Região de carregamento.

As integrais do domínio são:

$$
\int_{\Omega_{g}} g(q) w^{*}(s, q) d \Omega_{g}(q)
$$




$$
\int_{\Omega_{\mathrm{g}}} \mathrm{g}(\mathrm{q}) \frac{\partial \mathrm{w}^{*}(\mathrm{~s}, \mathrm{q})}{\partial m} \mathrm{~d} \Omega_{\mathrm{g}}(\mathrm{q})
$$

Em que,

$$
\begin{aligned}
& \mathrm{w}^{*}=\frac{\mathrm{r}^{2}}{8 \pi D}(\ln \mathrm{r}-1 / 2) \\
& \frac{\partial \mathrm{w}^{*}}{\partial \mathrm{m}}=-\frac{\mathrm{r}}{4 \pi D} \ln \mathrm{r} \cos \varphi
\end{aligned}
$$

De acordo com a Figura 3.5, pode-se escrever g, em relação ao sistema de coordenadas $(x, y)$ :

$$
g(q)=A x(q)+B y(q)+C
$$

Em relação ao sistema de coordenadas $(\bar{x}, \bar{y})$, de origem em s (Figura 3.5):

$$
\begin{aligned}
& x(q)=x(s)+\bar{x}(q) \\
& y(q)=y(s)+\bar{y}(q)
\end{aligned}
$$

E substituindo agora na equação (3.79):

$$
g(q)=A x(q)+B y(q)+C+A \bar{x}(q)+B \bar{y}(q)=g(s)+A \bar{x}(q)+B \bar{y}(q)
$$

Onde, g(s) é o valor da carga g no ponto s.

Substituindo a expressão (3.82) na equação (3.75), realiza-se a transformação em coordenadas polares: 


$$
\int_{\Omega_{\mathrm{g}}} \mathrm{g}(\mathrm{q}) \mathrm{w}^{*}(\mathrm{~s}, \mathrm{q}) \mathrm{d} \Omega_{\mathrm{g}}(\mathrm{q})=\int_{\Omega_{\mathrm{g}}}[\mathrm{g}(\mathrm{s})+\operatorname{Ar} \cos \theta+\operatorname{Br} \operatorname{sen} \theta] \mathrm{w}^{*}(\mathrm{~s}, \mathrm{q}) \mathrm{d} \Omega_{\mathrm{g}}(\mathrm{q})
$$

Substituindo a equação (3.77) na equação (3.83), e sendo g(s) uma constante e $\mathrm{d} \Omega_{\mathrm{g}}=\mathrm{rdr} \mathrm{d} \theta$ (Figura 3.5), tem-se:

$$
\begin{aligned}
\int_{\Omega_{g}} g(q) w^{*}(s, q) & d \Omega_{g}(q) \\
= & \frac{g(s)}{8 \pi D} \int_{\theta}\left[\int_{0}^{R} r^{3}(\ln r-1 / 2) d r\right] d \theta \\
& +\frac{1}{8 \pi D} \int_{\theta}\left[\int_{0}^{R}(A \cos \theta+B \operatorname{sen} \theta) r^{4}(\ln r-1 / 2) d r\right] d \theta
\end{aligned}
$$

Da integração em r, com o ângulo $\theta$ constante:

$$
\begin{aligned}
& \int_{\Omega_{\mathrm{g}}} \mathrm{g}(\mathrm{q}) \mathrm{w}^{*}(\mathrm{~s}, \mathrm{q}) \mathrm{d} \Omega_{\mathrm{g}}(\mathrm{q}) \\
& =\frac{\mathrm{g}(\mathrm{s})}{32 \pi \mathrm{D}} \int_{\theta} \mathrm{R}^{4}(\ln \mathrm{R}-3 / 4) \mathrm{d} \theta \\
& +\frac{1}{40 \pi \mathrm{D}} \int_{\theta} \mathrm{R}^{5}(\mathrm{~A} \cos \theta+\mathrm{B} \operatorname{sen} \theta)(\ln \mathrm{R}-0,7) \mathrm{d} \theta
\end{aligned}
$$

A partir da Figura 3.5, escreve-se:

$$
\mathrm{d} \Gamma_{\mathrm{g}} \cdot \cos \beta=\mathrm{R} \cdot \mathrm{d} \theta
$$

Ou ainda,

$$
\mathrm{d} \theta=\frac{\mathrm{d} \Gamma_{\mathrm{g}} \cdot \cos \beta}{\mathrm{R}}
$$

Substituindo a equação (3.87) na equação (3.85) obtém-se a integral de domínio, transformada numa integral sobre o contorno do carregamento $\Gamma_{\mathrm{g}}$ : 


$$
\begin{aligned}
\int_{\Omega_{\mathrm{g}}} \mathrm{g}(\mathrm{q}) \mathrm{w}^{*}(\mathrm{~s}, \mathrm{q}) \mathrm{d} \Omega_{\mathrm{g}}(\mathrm{q}) \\
=\frac{\mathrm{g}(\mathrm{s})}{32 \pi \mathrm{D}} \int_{\Gamma_{\mathrm{g}}} \mathrm{R}^{3}\left(\ln \mathrm{R}-\frac{3}{4}\right) \cos \beta \mathrm{d} \Gamma_{\mathrm{g}} \\
+\frac{1}{40 \pi \mathrm{D}} \int_{\Gamma_{\mathrm{g}}} \mathrm{R}^{4}(\mathrm{~A} \cos \theta+\mathrm{B} \operatorname{sen} \theta)(\ln \mathrm{R}-0,7) \cos \beta \mathrm{d} \Gamma_{\mathrm{g}}
\end{aligned}
$$

De forma análoga ao procedimento feito para a equação (3.75) que resultou na equação (3.88), faz-se para a equação (3.76), resultando em:

$$
\begin{aligned}
\int_{\Omega_{\mathrm{g}}} \mathrm{g}(\mathrm{q}) \frac{\partial \mathrm{w}^{*}(\mathrm{~s}, \mathrm{q})}{\partial m} & \mathrm{~d} \Omega_{\mathrm{g}}(\mathrm{q}) \\
& =-\frac{\mathrm{g}(\mathrm{s})}{12 \pi \mathrm{D}} \int_{\Gamma_{\mathrm{g}}} \mathrm{R}^{2}\left(\ln \mathrm{R}-\frac{1}{3}\right) \cos \varphi \cos \beta \mathrm{d} \Gamma_{\mathrm{g}} \\
& -\frac{1}{16 \pi \mathrm{D}} \int_{\Gamma_{\mathrm{g}}} \mathrm{R}^{3}(\mathrm{~A} \cos \theta+\mathrm{B} \operatorname{sen} \theta)(\ln \mathrm{R}-0,25) \cos \varphi \cos \beta \mathrm{d} \Gamma_{\mathrm{g}}
\end{aligned}
$$


O objetivo deste capítulo é apresentar uma formulação do Método dos Elementos de Contorno para análise de pavimentos. Considerando pavimentos, temos o elemento de placa em associação com outros elementos estruturais que, no presente trabalho, é o elemento de viga (ou o conjunto denominado de grelhas).

A interação placa-viga desenvolvida neste trabalho se fez na forma da discretização da placa em elementos de contorno, acoplando-se estes a elementos de viga (Figura 4.1) representados pelas equações diferenciais ordinárias.



Figura 4.1 - Interação elementos de viga x placa.

Para uma placa com vigas concêntricas a seguinte equação integral pode ser escrita utilizando a formulação apresentada no capítulo anterior, na qual a contribuição da viga é substituída pelos seus esforços de interação: 


$$
\begin{aligned}
K(s) w(s) & +\int_{\Gamma}\left[\mathrm{q}_{\mathrm{n}}^{*}(\mathrm{~s}, \mathrm{Q}) \mathrm{w}(\mathrm{Q})-\mathrm{m}_{\mathrm{n}}^{*}(\mathrm{~s}, \mathrm{Q}) \frac{\partial w}{\partial n}(Q)-\mathrm{m}_{\mathrm{ns}}^{*}(\mathrm{~s}, \mathrm{Q}) \frac{\partial w}{\partial s}(Q)\right] d \Gamma(\mathrm{Q}) \\
& =\int_{\Gamma}\left[\mathrm{V}_{\mathrm{n}}(\mathrm{Q}) \mathrm{w}^{*}(\mathrm{~s}, \mathrm{Q})-\mathrm{m}_{\mathrm{n}}(\mathrm{Q}) \frac{\partial w^{*}}{\partial n}(\mathrm{~s}, \mathrm{Q})\right] d \Gamma(\mathrm{Q})+\sum_{i=1}^{N_{c}} R_{c_{i}}(Q) w_{c i}^{*}(\mathrm{~s}, \mathrm{Q}) \\
& +\int_{\Omega_{\mathrm{g}}} \mathrm{g}(\mathrm{Q}) \mathrm{w}^{*}(\mathrm{~s}, \mathrm{Q}) \mathrm{d} \Omega_{\mathrm{g}}(\mathrm{Q})+\int_{\mathrm{VIGAS}} \mathrm{P}_{3}(\mathrm{Q}) \mathrm{w}^{*}(\mathrm{~s}, \mathrm{Q}) \mathrm{dS}_{\mathrm{V}}(\mathrm{Q}) \\
& +V_{i}(Q) w_{i}^{*}(s, Q)-M_{i}(Q) \frac{\partial w_{i}^{*}}{\partial \mathrm{S}_{\mathrm{V}}}(\mathrm{s}, \mathrm{Q})-V_{k}(Q) w_{k}^{*}(s, Q) \\
& +M_{k}(Q) \frac{\partial w_{k}^{*}}{\partial \mathrm{S}_{\mathrm{V}}}(\mathrm{s}, \mathrm{Q})
\end{aligned}
$$

Sendo, $K(s)=1$ para um ponto interno $\mathrm{s} ; K(s)=\beta / 2 \pi$ para um ponto $\mathrm{S}$ no canto de placa com ângulo interno $\beta ; K(s)=1 / 2$ para um ponto $\mathrm{S}$ em um contorno suave.

E ainda, $R_{c_{i}}=m_{n s}^{-}-m_{n s}^{+}$, é a reação de canto da placa.

Onde: $w, \mathrm{~m}_{\mathrm{n}}$ e $\mathrm{V}_{\mathrm{n}}$ são, respectivamente, o deslocamento transversal, 0 momento fletor e a força cortante equivalente ao longo do contorno; $g(q)$ e $\Omega_{\mathrm{g}}$ são o carregamento transversal e a superfície onde é aplicado; $P_{3}(Q), V_{i}(Q), V_{k}(Q), M_{i}(Q)$ e $\mathrm{M}_{\mathrm{k}}(\mathrm{Q})$ são os esforços na interface placa-viga (conforme Figura 4.1) e $\mathrm{S}_{\mathrm{V}}$ é uma coordenada ao longo de cada elemento de viga. O símbolo * é usado para indicar soluções fundamentais. Nesta equação $R_{c_{i}}$ é a reação de canto de placa.

Como visto no capítulo anterior, da equação (4.1) pode-se obter a seguinte equação integral da derivada do deslocamento com relação à direção $\mathrm{m}_{\mathrm{s}}$ de um sistema de coordenadas $\left(\mathrm{m}_{\mathrm{s}}, \mathrm{u}_{\mathrm{s}}\right)$ : 


$$
\begin{aligned}
K_{1}(S) \frac{\partial w}{\partial m_{s}}(S)+ & K_{2}(S) \frac{\partial w}{\partial u_{s}}(S) \\
& +\int_{\Gamma}\left[\frac{\partial \mathrm{q}_{\mathrm{n}}^{*}(\mathrm{~S}, \mathrm{Q})}{\partial m_{s}} \mathrm{w}(\mathrm{Q})-\frac{\partial \mathrm{m}_{\mathrm{n}}^{*}(\mathrm{~S}, \mathrm{Q})}{\partial m_{s}} \frac{\partial w}{\partial n}(Q)-\frac{\partial \mathrm{m}_{\mathrm{ns}}^{*}(\mathrm{~S}, \mathrm{Q})}{\partial m_{s}} \frac{\partial w}{\partial s}(Q)\right] d \Gamma(\mathrm{Q}) \\
& =\int_{\Gamma}\left\{\mathrm{V}_{\mathrm{n}}(\mathrm{Q}) \frac{\partial \mathrm{w}^{*}(\mathrm{~S}, \mathrm{Q})}{\partial m_{s}}-\mathrm{m}_{\mathrm{n}}(\mathrm{Q}) \frac{\partial}{\partial m_{s}}\left[\frac{\partial w^{*}}{\partial n}(\mathrm{~S}, \mathrm{Q})\right]\right\} d \Gamma(\mathrm{Q}) \\
& +\sum_{i=1}^{N_{c}} R_{c_{i}}(Q) \frac{\partial \mathrm{w}_{\mathrm{ci}}^{*}(\mathrm{~S}, \mathrm{Q})}{\partial m_{s}}+\int_{\Omega_{\mathrm{g}}} \mathrm{g}(\mathrm{q}) \frac{\partial \mathrm{w}^{*}(\mathrm{~S}, \mathrm{q})}{\partial m_{s}} \mathrm{~d} \Omega_{\mathrm{g}}(\mathrm{q}) \\
& +\int_{\mathrm{VIGAS}} \mathrm{P}_{3}(\mathrm{Q}) \frac{\partial \mathrm{w}^{*}(\mathrm{~s}, \mathrm{Q})}{\partial m_{s}} \mathrm{dS}_{\mathrm{V}}(\mathrm{Q})+V_{i}(Q) \frac{\partial \mathrm{w}_{\mathrm{i}}^{*}(\mathrm{~s}, \mathrm{Q})}{\partial S_{V}} \\
& -M_{i}(Q) \frac{\partial^{2} w_{i}^{*}}{\partial \mathrm{S}_{V}^{2}}(\mathrm{~s}, \mathrm{Q})-V_{k}(Q) \frac{\partial \mathrm{w}_{\mathrm{k}}^{*}(\mathrm{~s}, \mathrm{Q})}{\partial S_{V}}+M_{k}(Q) \frac{\partial^{2} w_{k}^{*}}{\partial \mathrm{S}_{V}^{2}}(\mathrm{~s}, \mathrm{Q})
\end{aligned}
$$

Sendo, $K_{1}(S)$ e $K_{2}(S)$ conforme exposto nas equações (3.70) e (3.71) respectivamente. Em que, $\gamma$ é o ângulo entre os sistemas de coordenas $(n, s)$ e (m, u), conforme Figura 3.4 .

\subsection{Representação da Viga}

A viga neste trabalho é tratada através da solução da equação da linha elástica, particularizada para carregamento distribuído linearmente, fazendo necessário discretizá-la a fim de gerar o correto acoplamento entre os elementos de viga e os elementos de contorno de placa. A viga é dividida em elementos definidos por nós nas extremidades e um nó em seu ponto central (Figura 4.2) e a partir dos parâmetros nodais associados a ele $(w, \partial w / \partial s)$ é obtido a expressão da linha elástica.

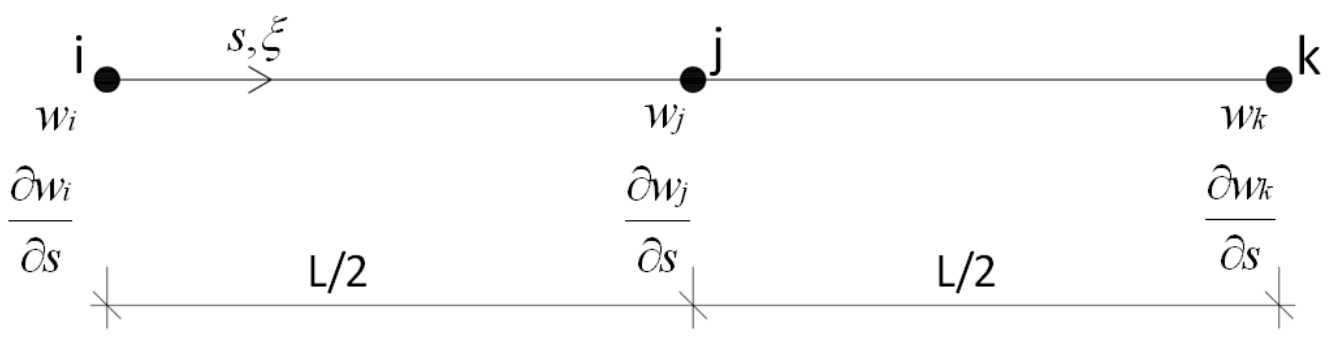

Figura 4.2 - Elemento de viga 
Considerando uma viga com seus elementos, pode-se representar a viga e seus elementos da seguinte forma:


Figura 4.3 - Conjunto de elementos de viga.

A expressão da elástica da viga é dada por:

$$
w(x)=w_{i} \alpha_{i 0}(x)+\frac{\partial w_{i}}{\partial s} \alpha_{i 1}(x) \operatorname{com} i=1, \ldots, n
$$

O momento fletor, a força cortante e o carregamento, estão respectivamente relacionados com a equação elástica da viga através das expressões:

$$
\begin{gathered}
M=-E I \frac{\partial^{2} w}{\partial s^{2}}(x) \\
V=-E I \frac{\partial^{3} w}{\partial s^{3}}(x) \\
\mathrm{P}_{3}=-E I \frac{\partial^{4} w}{\partial s^{4}}(\mathrm{x})
\end{gathered}
$$

Onde E é o módulo de elasticidade do material e I é o momento de inércia da viga.

Para gerar o acoplamento da placa (MEC) com a viga (EDO), a contribuição da viga na rigidez do conjunto placa-viga é substituída pelos esforços de interação presentes na interface destes dois elementos estruturais, a saber, o carregamento distribuído $P_{3}(Q)$, e os esforços de interface nas extremidades $V_{i}(Q)$, $\mathrm{V}_{\mathrm{k}}(\mathrm{Q}), \mathrm{M}_{\mathrm{i}}(\mathrm{Q})$ e $\mathrm{M}_{\mathrm{k}}(\mathrm{Q})$ (Figura 4.1). 
Nesta etapa do trabalho não está sendo considerada a torção da viga e assim somente os parâmetros nodais da viga referentes à flexão estão sendo utilizados (Figura 4.1). A cada nó são associados dois parâmetros nodais deslocamento vertical e sua derivada ao longo do eixo da viga (w e $\partial w / \partial s_{v}$ ). Assim no total têm-se seis parâmetros nodais.

Para este número de parâmetros nodais a elástica da viga pode ser aproximada por um polinômio do quinto grau, o que corresponde a um carregamento distribuído na interface variando linearmente ao longo do elemento de viga $\left(E I \frac{\partial^{4} w}{\partial x^{4}}=\right.$ $\left.\mathrm{P}_{3}\right)$.

Considerando o elemento finito de viga de comprimento $L$, e sendo a coordenada adimensional ao longo do eixo $s_{v}$ da viga, com origem no nó i da viga dada por:

$$
\xi=s_{v} / L
$$

Essa coordenada esta representada na Figura 4.2, e podemos assumir para o polinômio de $5^{\circ}$ grau as seguintes equações:

$$
w=\left[\begin{array}{llllll}
\xi^{5} & \xi^{4} & \xi^{3} & \xi^{2} & \xi & 1
\end{array}\right] .\{\alpha\}
$$

E sua derivada:

$$
\frac{\partial w}{\partial s}=\frac{1}{L} \cdot\left[\begin{array}{llllll}
5 \xi^{4} & 4 \xi^{3} & 3 \xi^{2} & 2 \xi & 1 & 0
\end{array}\right] .\{\alpha\}
$$

Impondo as condições de contorno nos nós do elemento obtêm-se:

$$
\left\{\begin{array}{c}
w_{i} \\
\partial w_{i} / \partial s \\
w_{j} \\
\partial w_{j} / \partial s \\
w_{k} \\
\partial w_{k} / \partial s
\end{array}\right\}=\left[\begin{array}{cccccc}
0 & 0 & 0 & 0 & 0 & 1 \\
0 & 0 & 0 & 0 & 1 / L & 0 \\
1 / 3 L & 1 / 16 & 1 / 8 & 1 / 4 & 1 / 2 & 1 \\
5 / 16 L & 1 / 2 L & 3 / 4 L & 1 / L & 1 / L & 0 \\
1 & 1 & 1 & 1 & 1 & 1 \\
5 / L & 4 / L & 3 / L & 2 / L & 1 & 0
\end{array}\right] \cdot\left\{\begin{array}{c}
\alpha_{1} \\
\alpha_{2} \\
\alpha_{3} \\
\alpha_{4} \\
\alpha_{5} \\
\alpha_{6}
\end{array}\right\}
$$

Da relação, $\{\delta\}=[c] .\{\alpha\}$, isolamos $\{\alpha\}$ :

$$
\{\alpha\}=[c]^{-1} \cdot\{\delta\}
$$


Substituindo na equação elástica da viga, ficamos com:

$$
w=[\phi] \cdot[c]^{-1} \cdot\{\delta\}=\left[\begin{array}{llllll}
\phi_{1} & \phi_{2} & \phi_{3} & \phi_{4} & \phi_{5} & \phi_{6}
\end{array}\right] \cdot\left\{\delta_{\mathrm{e}}\right\}
$$

Assim, a elástica da viga pode ser escrita em função dos parâmetros nodais da seguinte forma:

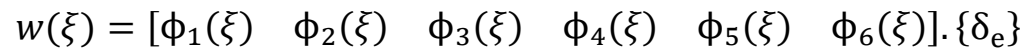

Onde $\left\{\delta_{\mathrm{e}}\right\}$ é o vetor de parâmetros nodais da viga e é dado por:

$$
\left\{\delta_{\mathrm{e}}\right\}^{\mathrm{T}}=\left[\begin{array}{llllll}
w_{i} & \frac{\mathrm{d} w_{i}}{\mathrm{ds}} & w_{j} & \frac{\mathrm{d} w_{j}}{\mathrm{ds}} & w_{k} & \frac{\mathrm{d} w_{k}}{\mathrm{ds}}
\end{array}\right]
$$

Portanto, as funções de forma $(\phi(\xi))$ são respectivamente:

$$
\begin{gathered}
\phi_{1}=24 \xi^{5}-68 \xi^{4}+66 \xi^{3}-23 \xi^{2}+1 \\
\phi_{2}=L \cdot\left(4 \xi^{5}-12 \xi^{4}+13 \xi^{3}-6 \xi^{2}+\xi\right) \\
\phi_{3}=16 \xi^{4}-32 \xi^{3}+16 \xi^{2} \\
\phi_{4}=L .\left(16 \xi^{5}-40 \xi^{4}+32 \xi^{3}-8 \xi^{2}\right) \\
\phi_{5}=-24 \xi^{5}+52 \xi^{4}-34 \xi^{3}+7 \xi^{2} \\
\phi_{6}=L \cdot\left(4 \xi^{5}-8 \xi^{4}+5 \xi^{3}-\xi^{2}\right)
\end{gathered}
$$

Calculando as segundas derivadas teremos:

$$
\begin{gathered}
\frac{\partial^{2} \phi_{1}}{\partial \xi^{2}}=\frac{1}{L^{2}} \cdot\left(480 \xi^{3}-816 \xi^{2}+396 \xi-46\right) \\
\frac{\partial^{2} \phi_{2}}{\partial \xi^{2}}=\frac{1}{L} \cdot\left(80 \xi^{3}-144 \xi^{2}+78 \xi-12\right) \\
\frac{\partial^{2} \phi_{3}}{\partial \xi^{2}}=\frac{1}{L^{2}} \cdot\left(192 \xi^{2}-192 \xi+32\right)
\end{gathered}
$$




$$
\begin{gathered}
\frac{\partial^{2} \phi_{4}}{\partial \xi^{2}}=\frac{1}{L} \cdot\left(320 \xi^{3}-480 \xi^{2}+192 \xi-16\right) \\
\frac{\partial^{2} \phi_{5}}{\partial \xi^{2}}=\frac{1}{L^{2}} \cdot\left(-480 \xi^{3}+624 \xi^{2}-204 \xi+14\right) \\
\frac{\partial^{2} \phi_{6}}{\partial \xi^{2}}=\frac{1}{L} \cdot\left(80 \xi^{3}-96 \xi^{2}+30 \xi-2\right)
\end{gathered}
$$

Da mesma forma calcula-se a terceira derivada:

$$
\begin{gathered}
\frac{\partial^{3} \phi_{1}}{\partial \xi^{3}}=\frac{1}{L^{3}} \cdot\left(1440 \xi^{2}-1632 \xi+396\right) \\
\frac{\partial^{3} \phi_{2}}{\partial \xi^{3}}=\frac{1}{L^{2}} \cdot\left(240 \xi^{2}-288 \xi+78\right) \\
\frac{\partial^{3} \phi_{3}}{\partial \xi^{3}}=\frac{1}{L^{3}} \cdot(384 \xi-192) \\
\frac{\partial^{3} \phi_{4}}{\partial \xi^{3}}=\frac{1}{L^{2}} \cdot\left(960 \xi^{2}-960 \xi+192\right) \\
\frac{\partial^{3} \phi_{5}}{\partial \xi^{3}}=\frac{1}{L^{3}} \cdot\left(-1440 \xi^{2}+1248 \xi-204\right) \\
\frac{\partial^{3} \phi_{6}}{\partial \xi^{3}}=\frac{1}{L^{2}} \cdot\left(240 \xi^{2}-192 \xi+30\right)
\end{gathered}
$$

A última derivada necessária é quarta derivada que resulta em:

$$
\begin{gathered}
\frac{\partial^{4} \phi_{1}}{\partial \xi^{4}}=\frac{1}{L^{4}} \cdot(2880 \xi-1632) \\
\frac{\partial^{4} \phi_{2}}{\partial \xi^{4}}=\frac{1}{L^{3}} \cdot(480 \xi-288) \\
\frac{\partial^{4} \phi_{3}}{\partial \xi^{4}}=\frac{1}{L^{4}} \cdot(384), \quad \text { que é uma constante }
\end{gathered}
$$




$$
\begin{gathered}
\frac{\partial^{4} \phi_{4}}{\partial \xi^{4}}=\frac{1}{L^{3}} \cdot(1920 \xi-960) \\
\frac{\partial^{4} \phi_{5}}{\partial \xi^{4}}=\frac{1}{L^{4}} \cdot(-2880 \xi+1248) \\
\frac{\partial^{4} \phi_{6}}{\partial \xi^{4}}=\frac{1}{L^{3}} \cdot(480 \xi-192)
\end{gathered}
$$

Fazendo uso das equações (4.3) e (4.4), em que o momento fletor é dado por $M=-E I\left(\partial^{2} w / \partial s^{2}\right)$ e a força cortante: $V=-E I\left(\partial^{3} w / \partial s^{3}\right)$, e substituindo os valores das funções deduzidas nas equações (4.20) a (4.37) na equação (4.11), têm-se:

- Momento fletor no nó inicial do elemento:

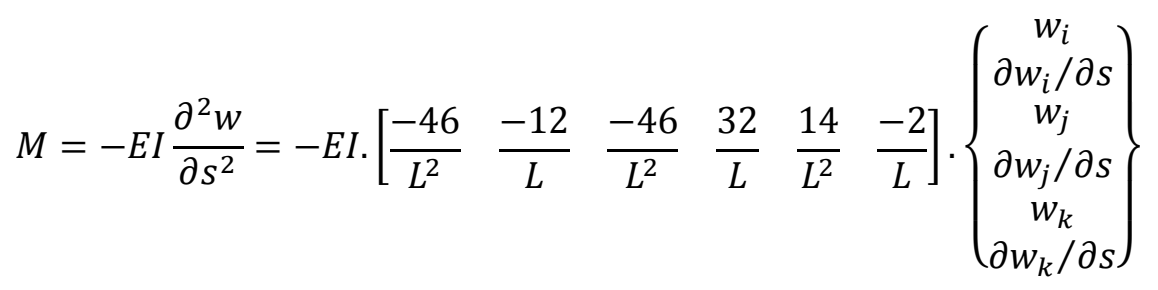

- Força cortante no nó inicial do elemento:

$$
V=-E I \frac{\partial^{3} w}{\partial s^{3}}=-E I \cdot\left[\begin{array}{llllll}
\frac{396}{L^{3}} & \frac{78}{L^{2}} & \frac{-192}{L^{3}} & \frac{192}{L^{2}} & \frac{-204}{L^{3}} & \frac{30}{L^{2}}
\end{array}\right] \cdot\left\{\begin{array}{c}
w_{i} \\
\partial w_{i} / \partial s \\
w_{j} \\
\partial w_{j} / \partial s \\
w_{k} \\
\partial w_{k} / \partial s
\end{array}\right\}
$$

- Momento fletor no nó final do elemento:

$$
M=-E I \frac{\partial^{2} w}{\partial s^{2}}=-E I \cdot\left[\begin{array}{llllll}
\frac{14}{L^{2}} & \frac{2}{L} & \frac{32}{L^{2}} & \frac{16}{L} & \frac{-46}{L^{2}} & \frac{12}{L}
\end{array}\right] \cdot\left\{\begin{array}{c}
\partial w_{i} \\
w_{j} \\
\partial w_{j} / \partial s \\
w_{k} \\
\partial w_{k} / \partial s
\end{array}\right\}
$$

- Força cortante no nó final do elemento: 


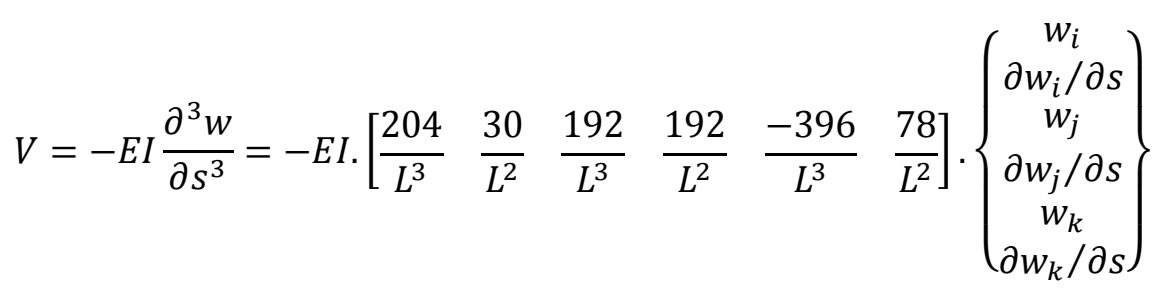

Representando os esforços calculados nas equações (4.38), (4.39), (4.40) e (4.41) no elemento de viga teremos o exposto na figura abaixo:



Figura 4.4 - Esforços no elemento de viga.

Os momentos são positivos para a placa, porém a cortante a esquerda é negativa, conforme Figura 4.2. Sendo este, o sinal que devem entrar para a montagem das equações.

\subsection{Acoplamento placa-viga}

Substituindo em (4.1) e (4.2) os esforços na interface placa-viga pelas suas expressões em função dos deslocamentos nodais da viga, obtêm-se uma equação integral em função dos deslocamentos e esforços nodais no contorno da placa e dos deslocamentos nodais dos elementos de viga.

Escrevendo as equações integrais do deslocamento $(w)$, e suas derivadas na direção normal $(\partial w / \partial n)$ e tangencial $(\partial w / \partial s)$ para todos os nós do contorno e dos elementos de viga do domínio e efetuando as integrais envolvidas, obtém-se o seguinte sistema de equações:

$$
\left[\begin{array}{ll}
H & H_{\Omega}
\end{array}\right] \cdot\left\{\begin{array}{l}
w_{\Gamma} \\
w_{\Omega}
\end{array}\right\}=[G] \cdot V_{\Gamma}+\{p\}
$$


onde,

$w_{\Omega}$ : vetor de valores nodais $\partial w / \partial x, \partial w / \partial y$ e $w$ de todos os nós da viga no domínio da placa e w, $\partial w / \partial n, \partial w / \partial s$ dos pontos no contorno da placa.

Como este sistema de equações tem mais incógnitas que equações, são escritas as equações integrais do deslocamento e suas derivadas para todos nós no domínio da placa, obtendo o seguinte sistema de equações:

$$
\left[\begin{array}{ll}
H^{*} & H_{\Omega}^{*}
\end{array}\right] \cdot\left\{\begin{array}{l}
w_{\Gamma} \\
w_{\Omega}
\end{array}\right\}=\left[G^{*}\right] \cdot V_{\Gamma}+\left\{p^{*}\right\}
$$

Combinando as equações (4.42) e (4.43), obtêm-se:

$$
\left[\begin{array}{ll}
H & H_{\Omega} \\
H^{*} & H_{\Omega}^{*}
\end{array}\right] \cdot\left\{\begin{array}{c}
w_{\Gamma} \\
w_{\Omega}
\end{array}\right\}=\left[\begin{array}{c}
G \\
G^{*}
\end{array}\right] \cdot V_{\Gamma}+\left\{\begin{array}{c}
p \\
p^{*}
\end{array}\right\}
$$

Para resolver todas as incógnitas do problema, impõem-se as condições de contorno, e o sistema dado na equação (4.44) fica:

$$
[A] .\{X\}=\{B\}
$$

Onde $\{\mathrm{X}\}$ é um vetor com as incógnitas do problema. Após a resolução deste sistema, deslocamentos e esforços são obtidos.

Uma vez obtidos os valores dos parâmetros nodais do contorno e do domínio, os deslocamentos em pontos do domínio são obtidos diretamente a partir da equação (4.2). 


\subsection{Introdução}

Com a formulação apresentada, desenvolveu-se um programa em FORTRAN possibilitando analisar exemplos de placas a fim de facilitar a avaliação da formulação. Depois de calculadas as placas com a formulação proposta, os resultados foram confrontados com resultados analíticos e com resultados obtidos via análise de elementos finitos, através do software ANSYS, em que as vigas foram modeladas com o elemento finito BEAM4 e as placas com o elemento SHELL63.

Visando demonstrar o processo de desenvolvimento evolutivo deste trabalho, os exemplos desenvolvidos partem de um modelo estrutural simplificado, considerando apenas uma placa simplesmente apoiada com vigas de bordo até a inserção de vigas no domínio, concluindo com exemplos de sistemas estruturais mais complexos com interação placa e viga tanto no contorno da placa como no domínio (pavimento de um edifício).

Abaixo, seguem os exemplos analisados que contribuíram para validar a formulação descrita neste trabalho. 
5.2 Exemplo 1 - Placa quadrada apoiada nos quatro cantos e vigas ao longo dos lados, com carregamento uniforme.

Neste exemplo analisou-se uma placa quadrada de lados de comprimento A, apoiada nos quatro cantos, Figura 5.1, com vigas ao longo dos lados com momentos de inércia $I_{V}=5 . A \cdot D / E$, sendo $\mathrm{D}$ a expressão (2.24) e submetida a um carregamento uniformemente distribuído q. Utilizou-se o coeficiente de poisson $v=0,25$ e dez elementos de contorno retos em cada lado da placa.

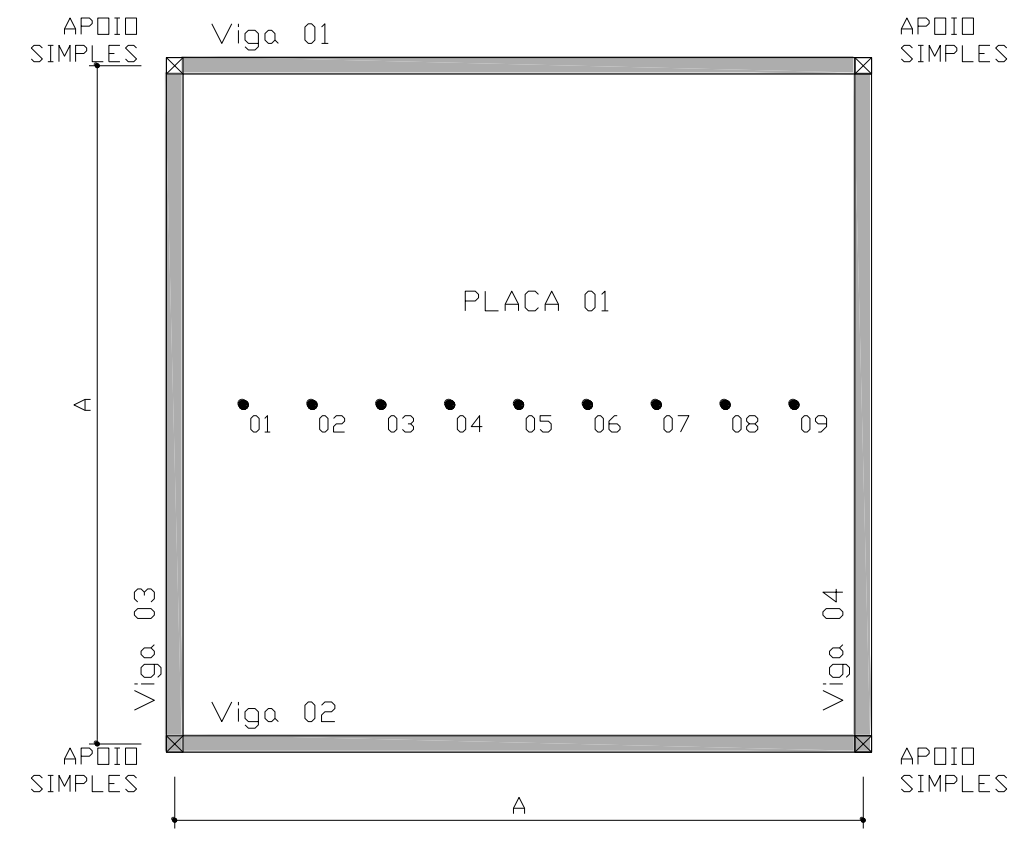

Figura 5.1 - Modelo de placa com viga nos bordos.

A Tabela 5.1 mostra os resultados obtidos quanto ao deslocamento vertical (w) pela formulação proposta, estes que foram comparados aos resultados analíticos encontrados em TIMOSHENKO e WOINOWSKY-KRIEGER (1970) e aos obtidos pelo Método dos Elementos Finitos através do software ANSYS 11.0 utilizando elementos SHELL 63, em sua discretização. Para a discretização em elementos finitos, utilizou-se de uma malha regular com 100 elementos. E para a discretização em elementos de contorno, adotou-se 40 elementos de contorno. 


\begin{tabular}{|c|c|c|c|}
\hline \multirow{2}{*}{ NÓ } & \multicolumn{3}{|c|}{ W (deslocamentos verticais) } \\
\cline { 2 - 4 } & TIMOSHENKO & MECXEDO & MEF \\
\hline 01 & & 0,002281 & 0,002265 \\
02 & & 0,003484 & 0,003460 \\
03 & & 0,004410 & 0,004381 \\
04 & & 0,004989 & 0,004957 \\
05 & 0,005190 & 0,005185 & 0,005153 \\
06 & & 0,004989 & 0,004957 \\
07 & & 0,004410 & 0,004381 \\
08 & & 0,003484 & 0,003460 \\
09 & & 0,002281 & 0,002265 \\
\hline
\end{tabular}

Tabela 5.1 - Resultados dos deslocamentos nodais

Observa-se que os valores de deslocamentos apresentam diferenças da ordem de $0,10 \%$ nos pontos do centro da placa entre os valores analíticos e 0,62 \% dos obtidos pelo Método dos Elementos Finitos. Apresentando, portanto, valores bem mais próximos dos valores analíticos quando comparado ao método dos elementos finitos.

Na Figura 5.2, apresenta-se os resultados obtidos para uma análise dos demais pontos de domínio, cuja sua representação gráfica foi obtida através do pós processamento feito no software SURFER 8.0.




Obtiveram-se também os esforços nas vigas (conforme Figura 5.3) de contorno da placa. Sendo seus resultados apresentados na tabela abaixo e confrontados com os obtidos pelo Método dos Elementos Finitos.

\begin{tabular}{|c|c|c|}
\hline \multirow{2}{*}{ NÓ } & \multicolumn{2}{|c|}{ Momentos fletores nas vigas } \\
\cline { 2 - 3 } 01 & MEC & MEF \\
\hline 03 & 0,000067 & 0,000018 \\
05 & 0,027408 & 0,027499 \\
07 & 0,042820 & 0,042955 \\
09 & 0,042820 & 0,042955 \\
11 & 0,027408 & 0,027499 \\
\hline
\end{tabular}

Tabela 5.2 - Momentos fletores nas vigas



Figura 5.3 - Viga de contorno da placa com seus respectivos nós.

Seguem os resultados da Tabela 5.2 plotados no gráfico abaixo:



Figura 5.4 - Gráfico de resultados dos momentos fletores nas vigas. 


\subsection{Exemplo 2 - Placa retangular apoiada nos quatro cantos e vigas ao longo dos lados e uma viga de domínio, com carregamento uniforme.}

O próximo exemplo é um problema prático. É um pavimento de um edifício com dimensões conforme Figura 5.5, o qual possui uma espessura constante de $10 \mathrm{~cm}$ e é submetido a uma carga uniforme de $6.8 \mathrm{kN} / \mathrm{m}^{2}$. Na análise numérica foi assumido que o pavimento é apoiado em seus 6 cantos. Para o concreto utilizado, adotou-se módulo de Young $\mathrm{E}=2000 \mathrm{kN} / \mathrm{cm}^{2}$ e coeficiente de Poisson $v=0,2$.



Figura 5.5 - Planta do modelo com uma viga no domínio.

A Tabela 5.3 mostra os resultados obtidos para o deslocamento vertical pela formulação proposta e sua comparação aos resultados obtidos pelo Método dos Elementos Finitos através do software ANSYS 11.0 utilizando 128 elementos SHELL 63, em sua discretização.

\begin{tabular}{|c|c|c|c|c|}
\hline \multirow{2}{*}{ Ponto } & \multicolumn{2}{|c|}{ Coordenadas } & \multicolumn{2}{c|}{ Deslocamento - w(cm) } \\
\cline { 2 - 5 } & $\mathbf{x}(\mathrm{cm})$ & $\mathbf{y}(\mathbf{c m})$ & MEC & MEF \\
\hline 01 & 0 & 175 & 0,10830 & 0,10730 \\
\hline 02 & 62,5 & 175 & 0,28182 & 0,28041 \\
\hline 03 & 125 & 175 & 0,41771 & 0,41673 \\
\hline 04 & 187,5 & 175 & 0,49646 & 0,49852 \\
\hline 05 & 250 & 175 & 0,51425 & 0,51358 \\
\hline 06 & 312,5 & 175 & 0,47605 & 0,47469 \\
\hline
\end{tabular}




\begin{tabular}{|l|c|c|c|c|}
\hline 07 & 375 & 175 & 0,39501 & 0,39219 \\
\hline 08 & 437,5 & 175 & 0,29865 & 0,29413 \\
\hline 09 & 500 & 175 & 0,24510 & 0,24022 \\
\hline 10 & 562,5 & 175 & 0,29865 & 0,29413 \\
\hline 11 & 625 & 175 & 0,39501 & 0,39447 \\
\hline 12 & 687,5 & 175 & 0,47605 & 0,47469 \\
\hline 13 & 750 & 175 & 0,51425 & 0,51358 \\
\hline 14 & 812,5 & 175 & 0,49646 & 0,49852 \\
\hline 15 & 875 & 175 & 0,41771 & 0,41673 \\
\hline 16 & 937,5 & 175 & 0,28182 & 0,28041 \\
\hline 17 & 1000 & 175 & 0,10830 & 0,10730 \\
\hline
\end{tabular}

Tabela 5.3 - Tabela com resultados de deslocamentos verticais para os nós da figura 5.3.

Observa-se que os valores de deslocamentos apresentam diferenças da ordem de 1,99\% nos pontos do centro da placa entre os valores obtidos pelo Método dos Elementos Finitos. Representando uma boa aproximação nos valores quando dos demais pontos observados no gráfico da Figura 5.6.



Figura 5.6 - Gráfico dos deslocamentos verticais da seção horizontal estudada.

O modelo analisado foi configurado com 28 nós, 24 elementos de contorno e 14 elementos de viga. A Figura 5.7 apresenta a locação nodal adotada. 


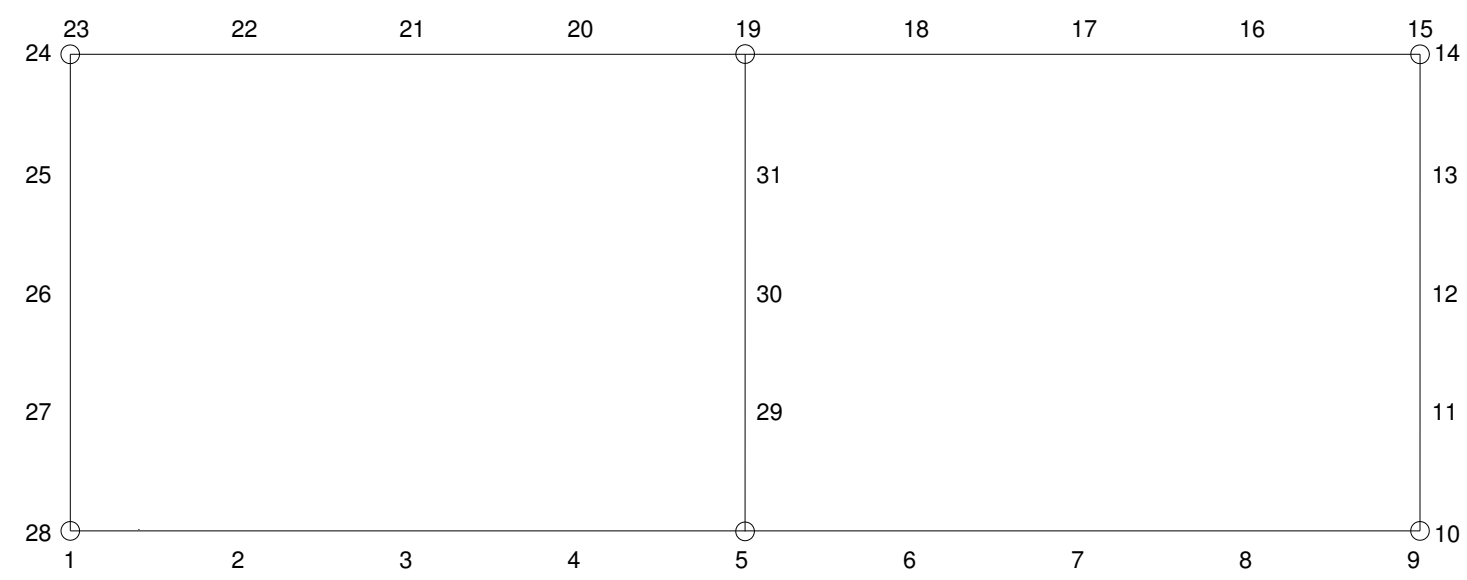

Figura 5.7 - Locação nodal dos elementos de contorno.

Na Figura 5.8, apresentam-se os resultados obtidos para uma análise dos demais pontos de domínio, cuja sua representação gráfica foi obtida através do pós processamento feito no software SURFER 8.0.



Figura 5.8 - Resultados de deslocamento em todos os pontos da placa via MEC 


\subsection{Exemplo 3 - Placa quadrada apoiada nos quatro cantos e vigas ao longo dos lados e do domínio, com carregamento uniforme.}

Neste novo exemplo, também é abordado um problema prático, porém é um pavimento com 4 nervuras em cada sentido, como estudado no trabalho de DIAS (2003). É um pavimento de um edifício com dimensões conforme planta representada na Figura 5.9, o qual possui uma espessura constante de $5 \mathrm{~cm}$ e é submetido a uma carga uniforme de $7.5 \mathrm{kN} / \mathrm{m}^{2}$. Na análise numérica assumiu-se que o pavimento é apoiado em seus 4 cantos. Para o concreto utilizado, adotouse módulo de Young $\mathrm{E}=2380 \mathrm{kN} / \mathrm{cm} 2$ e coeficiente de Poisson $v=0,2$. Dimensões da placa: $600 \mathrm{~cm} \times 600 \mathrm{~cm}$

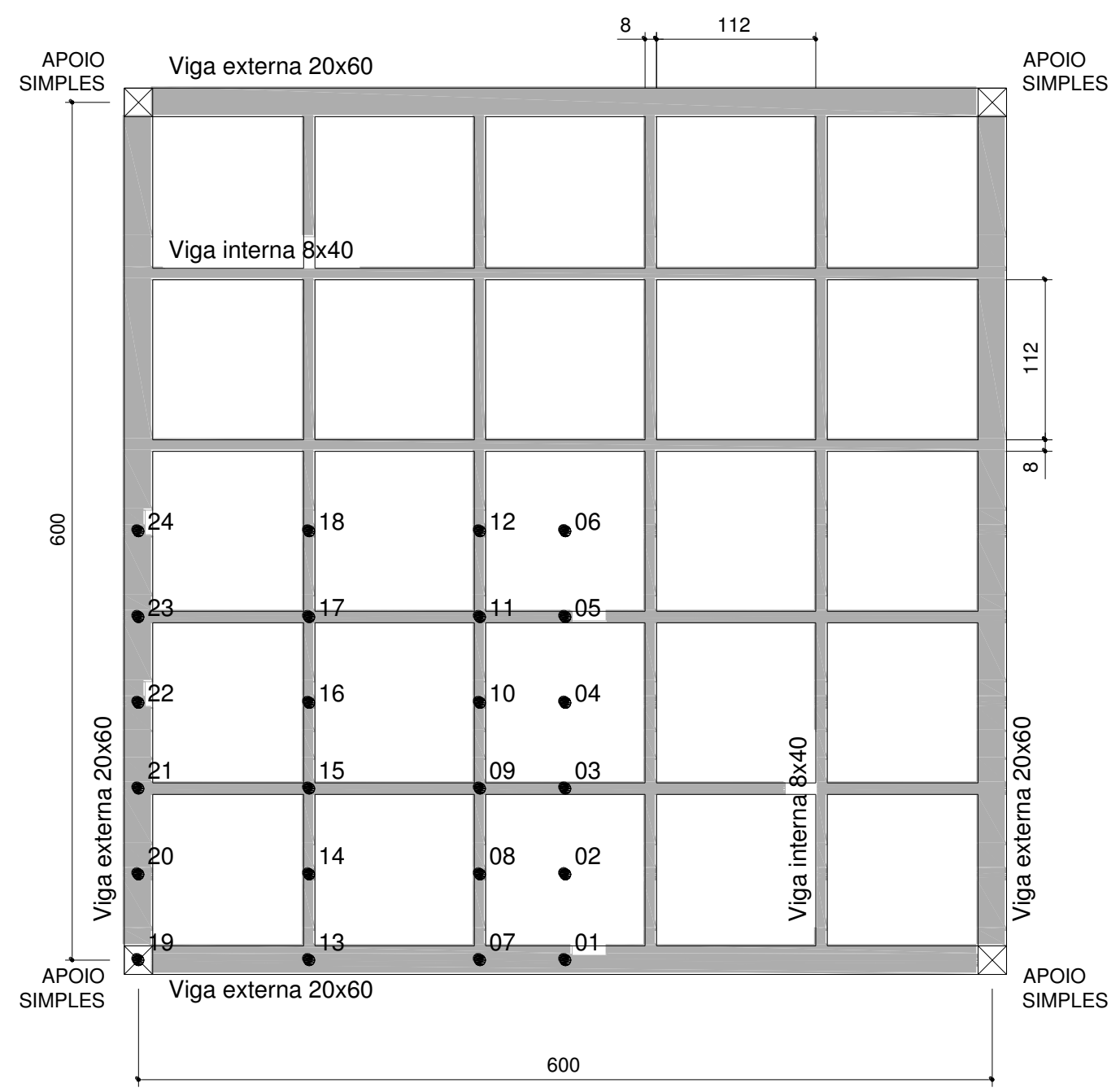

Figura 5.9 - Planta do pavimento com quatro nervuras em cada direção. 
A Tabela 5.4 mostra os resultados obtidos quanto a deslocamentos verticais $(w)$, pela formulação proposta e, comparados novamente aos resultados obtidos pelo Método dos Elementos Finitos através do software ANSYS 11.0. Utilizou-se 400 elementos regulares $(30 \mathrm{~cm}$ por $30 \mathrm{~cm})$ SHELL63 em sua discretização, especificados aqui por sua malha MEF 400. Utilizou-se também uma configuração de malhas no método de elementos finitos com 1600 elementos regulares $(15 \mathrm{~cm}$ por $15 \mathrm{~cm})$ SHELL63 especificados aqui por MEF 1600. Para a análise via Método dos elementos de contorno, o modelo foi configurado com 100 nós, 40 elementos de contorno e 60 elementos de viga.

\begin{tabular}{|c|c|c|c|c|c|}
\hline \multirow{2}{*}{ Ponto } & \multicolumn{2}{|c|}{ Coordenadas } & \multicolumn{3}{c|}{ Deslocamento - w(cm) } \\
\cline { 2 - 6 } & $\mathbf{x}(\mathbf{c m})$ & $\mathbf{y}(\mathbf{c m})$ & MEC & MEF 400 & MEF 1600 \\
\hline 1 & 300 & 0 & 0,26820 & 0,26478 & 0,26815 \\
\hline 2 & 300 & 60 & 0,54160 & 0,54084 & 0,54159 \\
\hline 3 & 300 & 120 & 0,76740 & 0,76508 & 0,76684 \\
\hline 4 & 300 & 180 & 0,95650 & 0,95421 & 0,95607 \\
\hline 5 & 300 & 240 & 1,06400 & 1,06100 & 1,06360 \\
\hline 6 & 300 & 300 & 1,11110 & 1,10790 & 1,11020 \\
\hline 7 & 240 & 0 & 0,25540 & 0,25513 & 0,25528 \\
\hline 8 & 240 & 60 & 0,51180 & 0,51039 & 0,51140 \\
\hline 9 & 240 & 120 & 0,73890 & 0,73663 & 0,73832 \\
\hline 10 & 240 & 180 & 0,91530 & 0,91237 & 0,91455 \\
\hline 11 & 240 & 240 & 1,02600 & 1,02310 & 1,02560 \\
\hline 12 & 240 & 300 & 1,06400 & 1,06100 & 1,06360 \\
\hline 13 & 120 & 0 & 0,15850 & 0,15840 & 0,15849 \\
\hline 14 & 120 & 60 & 0,35260 & 0,35155 & 0,35229 \\
\hline 15 & 120 & 120 & 0,52350 & 0,52191 & 0,52311 \\
\hline 16 & 120 & 180 & 0,65590 & 0,65387 & 0,65539 \\
\hline 17 & 120 & 240 & 0,73890 & 0,73663 & 0,73832 \\
\hline 18 & 120 & 300 & 0,76740 & 0,76508 & 0,76684 \\
\hline 19 & 0 & 0 & 0,00000 & 0,00000 & 0,00000 \\
\hline 20 & 0 & 60 & 0,08351 & 0,08342 & 0,08347 \\
\hline 21 & 0 & 120 & 0,15860 & 0,15840 & 0,15849 \\
\hline 22 & 0 & 180 & 0,21760 & 0,21737 & 0,21749 \\
\hline 23 & 0 & 240 & 0,25540 & 0,25513 & 0,25528 \\
\hline 24 & 0 & 300 & 0,26830 & 0,26800 & 0,26815 \\
\hline
\end{tabular}

Tabela 5.4 - Tabela com resultados de deslocamentos verticais para os nós da figura 5.7.

Observa-se que os valores de deslocamentos apresentam diferenças máximas da ordem de $0,10 \%$ para pontos no domínio da placa entre os valores 
obtidos pelo Método dos Elementos Finitos. Representando uma boa aproximação nos valores também observados nos gráficos da Figura 5.10.

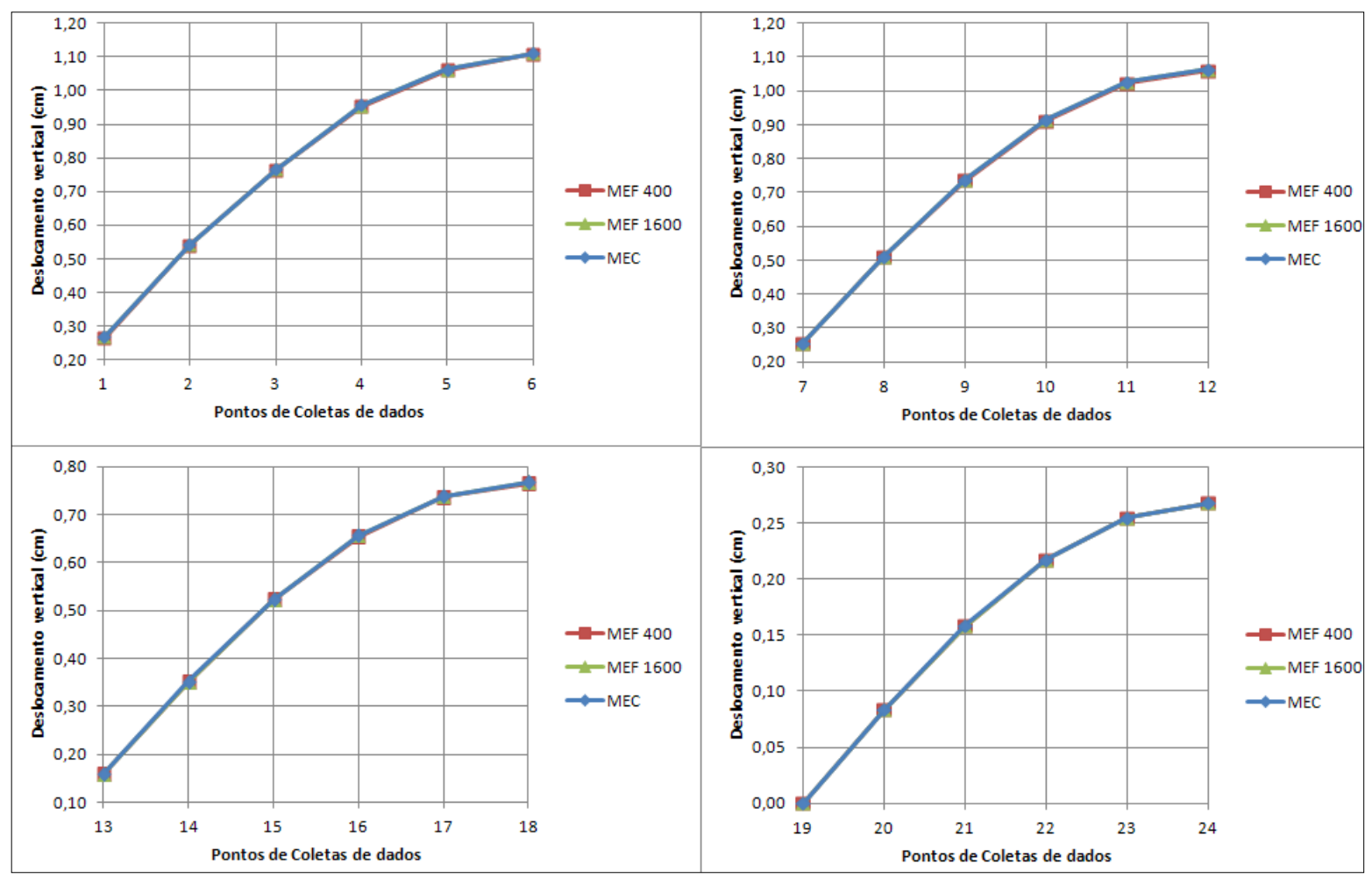

Figura 5.10 - Gráficos de deslocamentos para o modelo com nervuras.

Na Figura 5.11, apresentam-se os resultados obtidos para uma análise dos demais pontos de domínio, cuja sua representação gráfica foi obtida através do pós processamento feito no software SURFER 8.0.

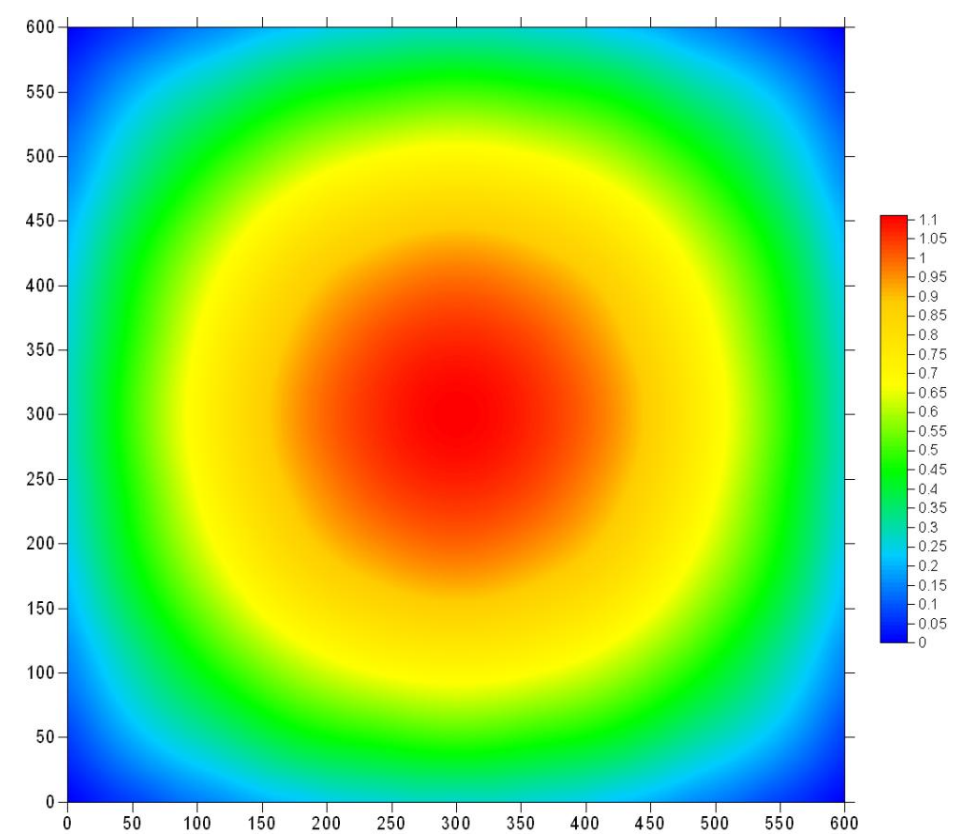

Figura 5.11 - Deslocamentos no domínio da placa nervurada. 
5.5 Exemplo 4 - Pavimento de um edifício com vigas ao longo dos lados e do domínio, com carregamento uniforme.

O próximo exemplo é finalmente um pavimento real de um edifício conforme a distribuição e dimensões de vigas e lajes da planta da Figura 5.12 (dimensões expressas em centímetros). Submetido a uma carga uniforme de $7.5 \mathrm{kN} / \mathrm{m}^{2}$. Para o concreto utilizado, adotou-se módulo de Young $\mathrm{E}$ $=2380 \mathrm{kN} / \mathrm{cm}^{2}$ e coeficiente de Poisson $v=0,2$.

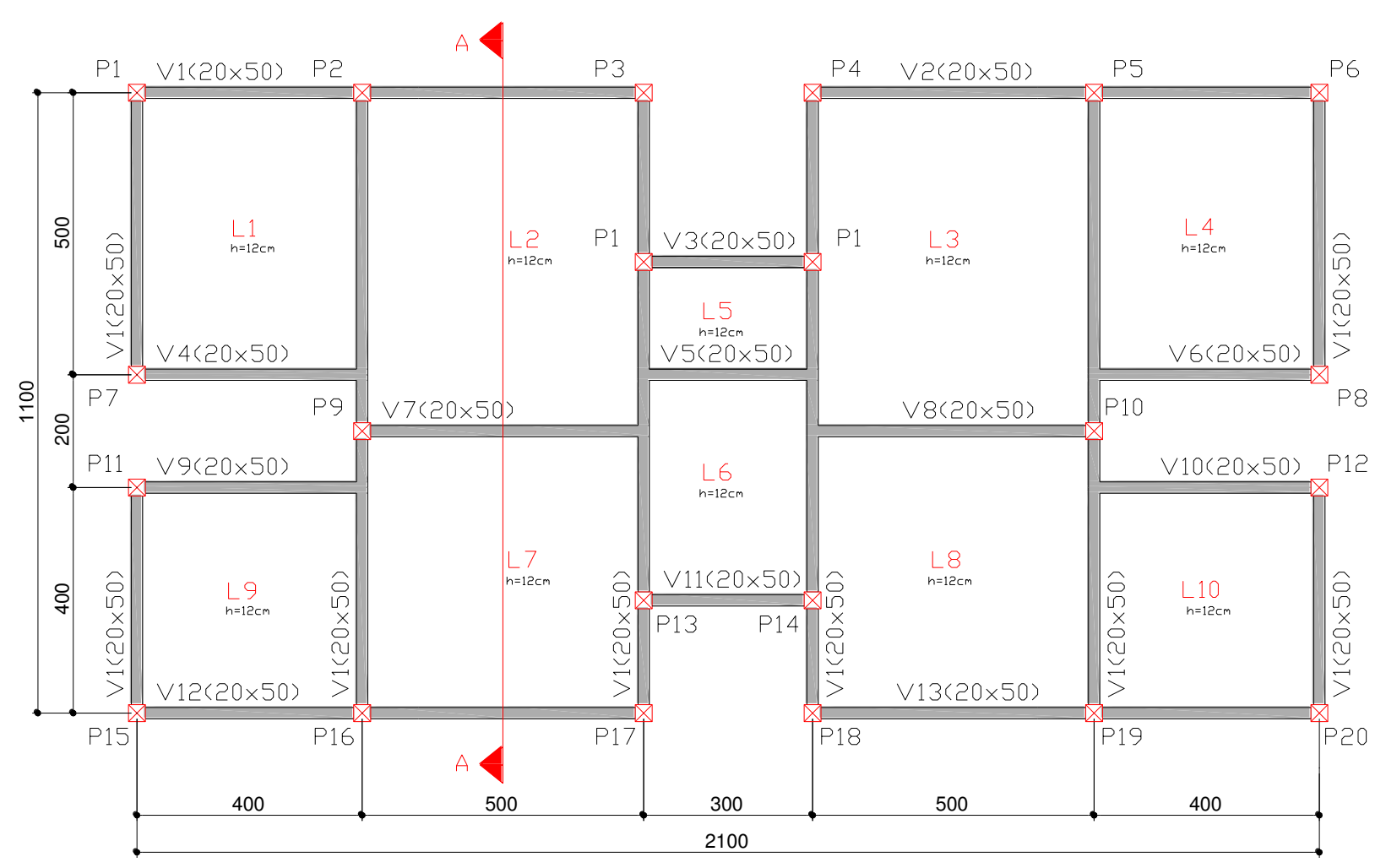

Figura 5.12 - Planta do pavimento tipo analisado.

A Tabela 5.5 mostra os resultados obtidos pela formulação proposta para o corte da seção A-A no pavimento e estes comparados novamente aos resultados obtidos pelo Método dos Elementos Finitos através do software ANSYS 11.0. Utilizou-se 400 elementos regulares $(50 \mathrm{~cm}$ por $50 \mathrm{~cm})$ SHELL63 em sua discretização, especificados aqui por sua malha MEF 400. Utilizou-se também uma configuração de malhas no método de elementos finitos com 1600 elementos regulares $(25 \mathrm{~cm}$ por $25 \mathrm{~cm})$ SHELL63 especificados aqui por MEF 1600 . Para a análise via Método dos elementos de contorno, o modelo foi configurado com 100 
elementos de contorno e 65 elementos de viga, representado nas tabelas e gráficos pela sigla MEC 100. Refinou-se os elementos de contorno para uma configuração com 200 elementos de contorno e 130 elementos de vigas (MEC 200).

\begin{tabular}{|c|c|c|c|c|c|c|}
\hline \multirow{2}{*}{ Ponto } & \multicolumn{2}{|c|}{ Coordenadas } & \multicolumn{4}{c|}{ Deslocamento - w(cm) } \\
\cline { 2 - 7 } & $\mathbf{x}(\mathbf{c m})$ & $\mathbf{y}(\mathbf{c m})$ & MEC 100 & MEC 200 & MEF 400 & MEF 1600 \\
\hline 1 & 750 & 0 & 0,06584 & 0,06857 & 0,06741 & 0,06799 \\
\hline 2 & 750 & 100 & 0,18565 & 0,21545 & 0,20519 & 0,20977 \\
\hline 3 & 750 & 200 & 0,26180 & 0,31289 & 0,29928 & 0,30526 \\
\hline 4 & 750 & 300 & 0,31382 & 0,37806 & 0,36930 & 0,37475 \\
\hline 5 & 750 & 400 & 0,34809 & 0,40323 & 0,40617 & 0,41148 \\
\hline 6 & 750 & 500 & 0,37455 & 0,39470 & 0,41315 & 0,41910 \\
\hline 7 & 750 & 600 & 0,43978 & 0,41371 & 0,44489 & 0,45131 \\
\hline 8 & 750 & 700 & 0,48435 & 0,42502 & 0,45508 & 0,46271 \\
\hline 9 & 750 & 800 & 0,48411 & 0,41928 & 0,43846 & 0,44762 \\
\hline 10 & 750 & 900 & 0,43548 & 0,38283 & 0,39764 & 0,40578 \\
\hline 11 & 750 & 1000 & 0,30075 & 0,27009 & 0,28009 & 0,28490 \\
\hline 12 & 750 & 1100 & 0,08200 & 0,07957 & 0,07970 & 0,08022 \\
\hline
\end{tabular}

Tabela 5.5 - Resultados nodais para seção de corte A-A.

Segue, na Figura 5.13, os resultados analisados graficamente:



Figura 5.13 - Gráfico com os resultados nodais para seção de corte A-A.

Para ambos os modelos, MEF e MEC, utilizou-se como alternativa aplicar a simetria geométrica e de carregamentos para modelar o pavimento analisado. Para a malha de elementos de contorno com 200 elementos (MEC 200), tem-se os 
deslocamentos dos demais pontos de domínio representados através do gráfico da Figura 5.14.

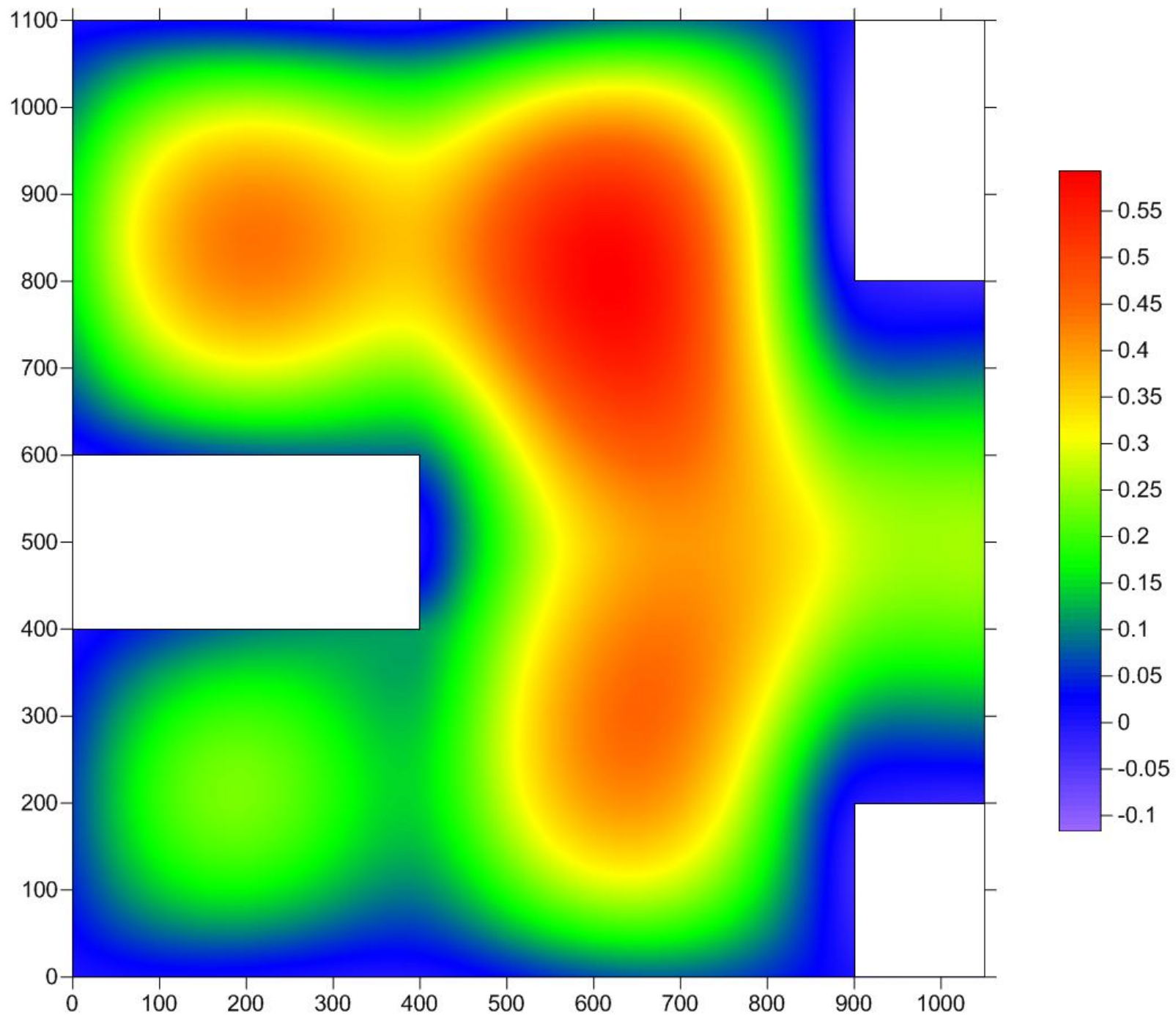

Figura 5.14 - Resultados de deslocamento em todos os pontos da placa via MEC 200. 
O objetivo deste trabalho foi o de apresentar uma formulação do método dos elementos de contorno para a análise de placas delgadas interagindo com elementos estruturais de viga, visando possibilitar a sua utilização prática para análise de pavimentos de edifícios.

A fim de compatibilizar adequadamente as vigas e as placa, utilizou-se da formulação do método dos elementos de contorno para placas delgadas em que se consideram três deslocamentos nodais para os nós do contorno da placa ( $\mathrm{w}, \partial \mathrm{w} / \partial \mathrm{n}$ e $\partial \mathrm{w} / \partial \mathrm{t}$ ) e a viga é substituída pelo seu efeito sobre a placa, através de um carregamento distribuído e forças de extremidades. A partir da resolução da equação diferencial de vigas os esforços da interação placa-viga são escritos em função dos parâmetros nodais da viga, isto é, we $\partial \mathrm{w} / \partial \mathrm{x}_{1}$.

A implementação da interação placa viga, utilizando para a viga as equações diferenciais ordinárias e para as placas três parâmetros nodais, apresenta significativa importância quanto à contribuição na melhoria dos resultados quando comparados com soluções analíticas e quando comparados ao método dos elementos finitos. A formulação proposta ainda apresenta outras vantagens, não incluídas neste trabalho, como a possibilidade de calcular os esforços nas vigas diretamente a partir da solução da equação diferencial e dos deslocamentos obtidos para seus nós.

Ressalta-se a facilidade de modelar as placas apoiadas em pontos distintos do contorno, pois apenas a divisão do contorno deve ser de tal forma que seus nós coincidam com os apoios. Também não é necessário dividir o contorno próximo ao apoio em grande número de elementos pois, mesmo com uma divisão regular, os resultados obtidos são muito bons.

Os problemas analisados no capítulo 5 demonstraram a correta formulação do problema. Iniciou-se comparando o primeiro exemplo com resultados de soluções analíticas, o que demonstrou a obtenção de resultados precisos através da formulação proposta. Evoluindo para modelos com vigas de domínio até um sistema estrutural em grelha com quatro nervuras em cada sentido no seu domínio, em que não houve divergências significativas quando seus resultados foram comparados ao MEF. No último exemplo analisado, que se considerou um pavimento real de um edifício, os resultados apresentados demonstraram um 
comportamento menos rígido para o modelo quando comparado ao método dos elementos finitos, expondo maiores deformações com o método estudado.

A formulação desenvolvida neste trabalho mostrou-se eficaz na análise dos problemas de pavimentos de edifícios na engenharia, demonstrando-se ser uma valiosa ferramenta de cálculo para o estudo destes problemas.

Para continuidade deste trabalho, como sugestão, é de grande importância o conhecimento dos esforços e momentos fletores nos pontos de domínio aqui analisados como também o estudo da influência da rigidez à torção da viga no acoplamento da placa com a viga. 
ARGYRIS, J.H.; KELSET, S. (1960). Energy theorems and structural analysis. London, Butteworths.

BARCELLOS, C.S.; SILVA, L.H.M. (1987). A boundary element formulation for the Mindlin's plate model. In: BREBBIA, C.A.; VENTURINI, W.S., eds. Boundary elements techniques: applications in stress analysis and heat transfer. Southampton, CMP.

BETTI, E. (1872). Teoria dell'elasticità. II Nuovo Ciemento, p.7-10.

BEZINE, G.P. (1978). Boundary integral formulation for plate flexure with arbitrary boundary conditions. Mech. Research Comm., v.5, n.4, p.197-206.

BEZINE, G.P. (1980). A mixed boundary integral-finite element approach to plate vibration problems. Mech. Res. Comm., v.7, p.141-150.

BEZINE,G.P. (1981). A boundary integral equation method for plate flexure with conditions inside the domain. Int. J. Num. Meth. Eng., v.17, p.1647-1657.

BEZINE, G.P.; GAMBY, D.A. (1978). A new integral equation formulation for plate bending problems. In: BREBBIA, C.A., ed. Recent advances in B.E.M. London, Pentech Press. p.327-342.

BEZINE, G.; GAMBY, D. (1982). Étude des mouvements transitoires de flexion d'une plaque par la méthode des équations intégrales de frontière. Journal de Mécanique Appliquée, v.1, n.3, p.451-466. 
CAMP, C.V.; GIPSON, G.S. (1990). Biharmonic analysis of rectilinear plates by boundary element method. Int. Num. Meth. Eng., n.30, p.517-539.

CHUEIRI, L.H.M. (1994). Formulação do método dos elementos de contorno para análise elastoplástica de placas. São Carlos. Tese (Doutorado) - Escola de Engenharia de São Carlos, Universidade de São Paulo.

COSTA JR., J.A.; BREBBIA, C.A. (1985). The boundary element method applied to plates on elastic foundations. Engineering Analysis, v.2, n.4, p.174-183.

COSTA JR., J.A. (1985). The boundary element method applied to plate problems. Southampton. Tese (Doutorado) - University of Southampton.

COSTA JR., J.A. (1988). Plate vibrations using B.E.M. Appl.Math.Modelling., v.12, p.78-85.

CRUSE, J.A.; RIZZO, F. (1968). A direct formulation and numerical solution of the general transient elastodynamic problems. Int. J. Math. Anal. Appl., v.22, p.244-259.

DANSON, D.J. (1979). Analysis of plate bending problems by direct boundary element method. Southampton, University of Southampton. (M.Sc. Dissertation)

DIAS, R. H. (2003). Análise numérica de pavimentos de edifícios em lajes nervuradas. Dissertação (Mestrado). São Carlos, SÃO PAULO: Escola de Engenharia de São Carlos, Universidade de São Paulo.

FERNANDES, G.R.; VENTURINI, W.S. (2005). Building floor analysis by the boundary element method, Computational mechanics, 35, pp. 277-291. 
HANSEN, E. B. (1976). Numerical solution of integro-differential and singular integral equations for plate bending problems. J. of Elasticity, v.6, n.1, p.39-56.

HARTLEY, G. A. (1996). Developments of plate bending elements for frame analysis. Engineering Analysis with Boundary Elements, v.17, p.93-104.

HARTLEY, G.A.; ABDEL-AKHER, A. (1988). Analytic integration procedures for plate bending analysis, in: BREBBIA, C.A. ed. Boundary elements X. C.M. Publ. v.3, p. 391-405.

HARTLEY, G.A.; ABDEL-AKHER, A. (1989). Boundary integration and interpolation procedures for plate bending. Int. Num. Meth. Engng. v.28(6), p.1389-1408.

HARTLEY, G. A.; ABDEL-AKHER, A. (1993). Analysis of building frames. J.

Struct. Eng., ASCE, v.119, p.468-483.

HARTLEY, G.A.; AHMED, A.A. (1989). Evaluation of boundary integrals for plate bending. Int. Num. Meth. Engng. v.28(2), p.75-93.

HARTLEY, G.A.; ABDEL-AKHER A.; CHEN P. (1992). Boundary element analysis of thin plates internally bounded by rigid patches, Int. J. for Numerical Methods in Engineering, v.35, p.1771-1785.

HE, X.; QIN, Q. (1993). Nonlinear analysis of Reissner's plate by the variational approaches and boundary element methods. Appl. Math. Modelling., v.17, n.3, p.149-155.

HU, C.; HARTLEY, G.A. (1994). Elastic analysis of thin plates with beam supports, Engineering Analysis with Boundary Elements 13, p. 229-238. 
JASWON, M. A.; MAITI, M.; SYMM, G. T. (1967). Numerical biharmonic analysis and some applications. Int. J. Solids Structures, Vol.3, p.309-332.

KAMIYA, N.; SAWAKY, Y. (1982). An integral equation approach to finite deflections of elastic plates. Int. J. Non-Linear Mech., v.17, n.3, p.187-194.

KANE, J.H. (1994). Boundary Element analysis in engineering continuum mechanics. Englewood Cliffs, N. J.: Prentice-Hall.

KARAM, V.J. (1986). Aplicação do método dos elementos de contorno à teoria de Reissner para flexão de placas. Rio de Janeiro. Dissertação (Mestrado) - COPPE, Universidade Federal do Rio de Janeiro.

KARAM, V.J.; TELLES, J.C.F. (1992). B.E.M. applied to plate bending elastoplastic analysis using Reissner's theory. Engineering Analysis with Boundary Elements, v.9, n.4, p.351-357.

KATSIKADELIS, J.T.; ARMENAKAS, A.E. (1984). Plates on elastic foundations by B.I.E. Method. J. Eng. Mech., ASCE, v.110, n.7, p.1086-1104.

KATSIKADELIS, J.T.; ARMENAKAS, A.E. (1989). A new boundary equation solution to the plate problem. J. Appl. Mech. v.56(2), p.364-374.

KIRCHHOFF, G. (1850). Über das Gleichgewicht und die Bewegung einer elastichen Scheibe. J. Math., v.40, p.51-58.

KITAHARA, M. (1985). Boundary integral equation method in eigenvalue problems of elastodynamics and thin plates. In: BREBBIA, C.A., ed. Topics in boundary element research. Berlin, Springer-Verlag., v.2. 
KUPRADZE, V.D. (1965). Potential methods in the theory of elasticity. Israel Program for Scientific Translations, Jerusalem.

LEONETTI ET AL. (2009). A symmetric boundary element model for the analysis of Kirchhoff plates.

LONG, S.Y.; BREBBIA, C.A.; TELLES, J.C.F. (1988). Boundary element bending analysis of moderately thick plates. Engineering Analysis, v.5, n.2, p.64-74.

MENDONÇA, A. V.; PAIVA, J.B. (2008). Boundary Element Analysis of Plate-Beam Interaction. In: XXXII Jornadas Sud Americanas de Ingenieria Estructural, 2008, Santiago. CD ROOM XXXII JORNADAS SUD AMERICANAS DE INGENIERIA ESTRUCTURAL.

MENDONÇA, A.V; PAIVA J.B. (2010). A coupled boundary element/differential equation method formulation for plate-beam interaction analysis. Engineering Analysis with Boundary Elements, v.34, p. 456-462.

MIKHLIN, S.G. (1957). Integral Equation. London, Pergamon Press.

MINDLIN, R.D. (1951). Influence of rotatory inertia and shear on flexural motions of isotropic, elastic plates. J. Appl. Mech., v.13, p.31-38.

MOSHAIOV, A.; VORUS, W.S. (1986). Elasto-plastic plate bending analysis by a boundary element method with initial plastic moments. Int. J. Solids Structures, v.22, n.11, p.1213-1229.

MUSKELISHVILI, N.I. (1953). Some basic problems of the mathematical theory of elasticity. Noordhoff, Gronigen Holand. 
NARDINI, D.; BREBBIA, C.A. (1982). A new approach of free vibration analysis using boundary elements. In: BREBBIA, C.A., ed. Boundary element method in engineering. Berlin, Springer-Verlag. p.312-326.

NG, S.F.; CHEUNG, M.S.; XU, T. (1989). Application of the combined boundary element and finite method to slab and slab-ongrider bridges. In: TANAKA, M.; BREBBIA, C.A.; HONMA, T., Eds. Boundary elements XII. Southampton, CMP.p.457-466.

NIWA, Y.; KOBAYASHI, S.; KITAHARA, M. (1981). Eigenfrequency analysis of a plate by the integral equations method. Theoretical and applied mechanics. Univ. of Tokyo Press, v.29, p.287-307.

NIWA, Y.; KOBAYASHI, S.; KITAHARA, M. (1982). Applications of the boundary integral method to eigenvalue problems of elastodynamics. In: BREBBIA, C.A., ed. Boundary element method in engineering. Berlin, Springer-Verlag. p.297-311.

OLIVEIRA NETO, L.; PAIVA, J.B. (2004). Cubic approximation for the transverse displacement in BEM for elastic plates analysis, Engineering Analysis with Boundary Elements, v.28, p.869-880, 2004.(ISSN: 0955-7997)

OLIVEIRA NETO, L. (1998). Uma formulação do método dos elementos de contorno com três parâmetros nodais em deslocamentos para placas delgadas e suas aplicações a problemas de engenharia estrutural. São Carlos. Tese (Doutorado)- Escola de Engenharia de São Carlos - Universidade de São Paulo.

PAIVA, J.B. (1987). Formulação do método dos elementos de contorno para a flexão de placas e suas aplicações em engenharia de estruturas. São Carlos. Tese (Doutorado) - Escola de Engenharia de São Carlos, Universidade de São Paulo. 
PAIVA, J.B. (1991). Boundary element formulation for plate analysis with special distribuition of reactions along the boundary. Adv. Eng. Soft. and Workst., v.13, p.162-168.

PAIVA, J.B. (1996). Boundary elements formulation for building slabs. Engineering Analysis with Boundary Elements, v.17, n.2, p.105-110.

PAIVA, J.B.; BUTTERFIELD, R. (1997). Boundary elements analysis of plate-soil interaction. Comp. \& Struct., v.64, n.1, p.319-328.

PAIVA, J.B.; MENDONÇA, A.V. (1997). Boundary element analysis of piled rafts. In: MARCHETTI, M.; BREBBIA, C.A.; ALIABADI, M.H., eds. Boundary elements XIX. Southampton, CMP. p.799-808.

PAIVA J.B.; MENDONÇA A.V. (2008). "A Boundary Element - Differential Equation Method Coupling for Plate-Beam Interaction", Proc. of the Ninth International Conference on Computational Structures Technology, Athens, Greece, 02-05 September, p.147.

PAIVA J.B.; MENDONÇA A.V. (2010). "A Coupled Boundary Element / Differential Equation Method Formulation for Plate-Beam Interaction Analysis", Engineering Analysis with Boundary Elements, Vol. 34, p.456-462.

PAIVA, J. B.; OLIVEIRA NETO, L. (1995). An alternative boundary element formulation for plate bending analysis. In: ALIABADI, M.H. et al., (Eds.). Boundary element technology $X$ (Proc. 10th International Conference on Boundary Element Technology, Liège, Belgium, Sep. 1995). Southampton, CMP. p.1-8. 
PAIVA, J.B.; TRONDI, R.R. (1996). A simplified boundary elements method analysis of pile groups.In: TOPPING, B.H.V., ed. Adv. In B.E.M,: Proc., Budapest, 1996. CivilComp Press, p.145-152.

PAIVA, J.B.; VENTURINI, W.S. (1985). Boundary element algorithm for building floor slab analysis. In: BREBBIA, C.A.; NOYE, B.J., ed. BETECH 85: Proc., Adelaide, 1985. Springer-Verlag.

PAIVA J.B.; VENTURINI, W.S. (1987). Analysis of building structures considering plate-beam-column interactions. In: BREBBIA, C.A.; VENTURINI, W.S., eds, Boundary element techniques. Southampton, CMP.

PARIS, F.; LEÓN, S. (1985). An alternative analysis of thin elastic plates with any boundary conditions, using B.E.M. In: VII Int. Conf. On Boundary Element Methods, 7., 1985. Proc. Springer Verlag. p.4-28.

PARIS, F.; LEÓN, S. (1987). Analysis of thermal stress in plates with boundary element method. Eng. Anal., v.4, p.199-203.

PROVIDAKIS, C.P.; BESKOS, D.E. (1989). Free and forced vibration of plates by boundary and interior elements. Int. J. Num. Meth. Eng., v.28, p.1977-1994.

REISSNER, E. (1944). On bending of elastic plates. J. Math. Physics., v.23, p.184191.

RIBEIRO, G.O. (1992). Sobre a formulação do método dos elementos de contorno de placas usando as hipóteses de Reissner. São Carlos. Tese (Doutorado) - Escola de Engenharia de São Carlos, Universidade de São Paulo. 
RIBEIRO, G.O.; VENTURINI, W.S. (1989). Aplicação do método dos elementos de contorno no cálculo de placas através da teoria de Reissner. In: CONGRESSO IBERO-LATINO-AMERICANO SOBRE MÉTODOS COMPUTACIONAIS EM ENGENHARIA, 10., Porto, Portugal, 1989. Anais. Porto, Faculdade de Engenharia do Porto. v.1, p.A389-398.

RIZZO, F.J. (1967). Na integral approach to boundary value problems of classical elastostatics. Quartely of Apllied Mathematics, v.25, n.1, p.83-92.

SAPOUNTZAKIS, E.J.; DIKAROS, I.C. (2012). Large deflection analysis of plates stiffened by parallel beams. Engineering Structures, v. 35, p. 254-271.

SAPOUNTZAKIS, E.J.; MOKOS, V.G.(2008). An improved model for the analysis of plates stiffened by parallel beams with deformable connection. Computers and Structures, v. 86, p. 2166-2181.

SAWAKI, Y.; TAKEUCHI, K.; KAMIYA, N. (1989). Finite deflection analysis of plates by dual reciprocity boundary elements. In: BREBBIA, C.A.; CONNOR, J.J., eds. Adv. In B.E., Southampton, CMP. v.3, p.145-152.

SAWAKI, Y.; TAKEUCHI, K.; KAMIYA, N. (1990). Nonlinear bending analysis of plates without domain-cell discretization. Engineering Analysis with Boundary Elements, v.7, n.3, p.130-135.

SILVA, N.A.; VENTURINI, W.S. (1988) Dual reciprocity process applied to solve bending plates on elastics foundations. In: BREBBIA, C.A., ed. Boundary elements $X$ : stress analysis. Southampton, CMP., v.3, p.95-105.

SOUTHWELL, R.V. (1946). Relaxation methods in theoretical physics. London, Oxford University Press. 
STERN, M. (1979). A general boundary integral formulations for the numerical solution of plate bending problems. Int. J. Sol. Struct., v.15, p. 769-782.

TANAKA, M.; YAMAGIWA, K.; MIYAZAKI, K.; UEDA, T. (1987). Integral equation approach to free vibration problems of assembled plate structures. In: TANAKA, M.; DUO, Q., eds. Theory and applications of boundary element methods. Oxford, Pergamon Press.

TEJERINA CALDERÓN, E.; VENTURINI, W.S. (1997). Interação de places com o meio contínuo através do método dos elementos de contorno. In: JORNADAS SUL AMERICANAS DE ENGENHARIA ESTRUTURAL, 28., São Carlos, 1997. Estruturas e Fundações. São Carlos, EESC-USP/ASAEE. v.3, p.1005-1014

TIMOSHENKO, S.P.; WOINOWSKY-KRIEGER, S. (1959). Theory of Plates and Shells. New York, McGraw-Hill.

TIMOSHENKO, S.P.; WOINOWSKY-KRIEGER, S. (1970). Teoria de places y laminas. Trad. F.J. Medina Salanova. Bilbao, España, Ediciones URMO.

TOTTENHAM, H. (1979). The boundary element method for plates and shells. In: BANERJEE, P.K.; BUTTERFIELD, R., eds. Developments boundary element methods 1. London, Applied Science Publ. p.173-205.

TURNER, M.J. et al. (1956). Stiffnes and deflection analysis of complex structures. J. Aero. Science, n.23, p.805-823.

VABLE, M.; ZHANG, Y. (1992). A boundary element method for plate bending problems. Int. J. Solids Structures, v.29, n.3, p.345-361. 
VAN DER WEEËN, F. (1982). Application of the direct boundary element method to Reissner's plate model. In: BREBBIA, C.A., ed. In: BETECH 82: Proc., Southampton, England, 1982. Springer- Verlag.

VENTSEL, E.S. (1997). An indirect boundary element method for plate bending analysis. Int. J Num. Meth. Eng., v.40, p.1597-1610.

VIVOLI, J.; FILIPPI, P. (1974). Eigenfrequencies of thin plates and layer potentials. J. Acoust. Soc. Am., v.55, p.562-567.

WAIDEMAM L.; VENTURINI W. (2009). "BEM Formulation for Reinforced Plates", Engineering Analysis with Boundary Elements, Vol.33, pp.830-836.

WAIDEMAM, L.; VENTURINI, W. (2009). "An Extended BEM Formulation for Plates Reinforced by Rectangular Beams”, Engineering Analysis with Boundary Elements, Vol. 33, p.983-992.

WEISS, O.; MOSHAIOV, A. (1993). Vibration analysis of continuous plate structures using boundary integrals. Comp. \& Struct., v.47, n.6, p.971-976.

WESTPHAL JR., T.; BARCELLOS, C.S. (1989). Applications of the boundary element method to Reissner's and Mindlin's plate models. In: TANAKA, M.; BREBBIA, C.A.; HONMA, T., eds. Boundary elements XII, Southampton, CMP. p.467-480.

WONG, G.K.K; HUTCHINSON, J.R. (1981). An improved boundary element method for plate vibrations. In: BREBBIA, C.A., ed. Boundary element methods. Berlin, Springer- Verlag. p.272-289. 
XIAO-YAN, L.; KUANG-MAO, H.; XIUXI, W. (1990). Geometrically nonlinear analysis of Reissner type plate by boundary element method. Comp. \& Struct., v.37, n.6, p.911-916. 